Portland State University

PDXScholar

Winter 3-16-2015

\title{
Technological Forecasting Based on Segmented Rate of Change
}

Dong-Joon Lim

Portland State University

Follow this and additional works at: https://pdxscholar.library.pdx.edu/open_access_etds

Part of the Operations Research, Systems Engineering and Industrial Engineering Commons, and the Technology and Innovation Commons

Let us know how access to this document benefits you.

\section{Recommended Citation}

Lim, Dong-Joon, "Technological Forecasting Based on Segmented Rate of Change" (2015). Dissertations and Theses. Paper 2220.

https://doi.org/10.15760/etd.2217

This Dissertation is brought to you for free and open access. It has been accepted for inclusion in Dissertations and Theses by an authorized administrator of PDXScholar. Please contact us if we can make this document more accessible: pdxscholar@pdx.edu. 
Technological Forecasting Based on Segmented Rate of Change

by

Dong-Joon Lim

A dissertation submitted in partial fulfillment of the requirements for the degree of

\author{
Doctor of Philosophy \\ in \\ Technology Management
}

Dissertation Committee:

Timothy R. Anderson, Chair

Tugrul U. Daim

Antonie J. Jetter

Wayne W. Wakeland

Portland State University

2015 
(C) 2015 Dong-Joon Lim 


\begin{abstract}
Consider the following questions in the early stage of new product development. What should be the target market for proposed design concepts? Who will be the competitors and how fast are they moving forward in terms of performance improvements? Ultimately, is the current design concept and targeted launch date feasible and competitive?

To answer these questions, there is a need to integrate the product benchmarking with the assessment of performance improvement so that analysts can have a risk measure for their R\&D target setting practices. Consequently, this study presents how time series benchmarking analysis can be used to assist scheduling new product releases. Specifically, the proposed model attempts to estimate the "auspicious" time by which proposed design concepts will be available as competitive products by taking into account the rate of performance improvement expected in a target segment.

The empirical illustration of commercial airplane development has shown that this new method provides valuable information such as dominating designs, distinct segments, and the potential rate of performance improvement, which can be utilized in the early stage of new product development. In particular, six dominant airplanes are identified with corresponding local RoCs and, inter alia, technological advancement toward long-range and wide-body airplanes represents very competitive segments of the market with rapid changes. The resulting individualized RoCs are able to estimate the arrivals of four different design concepts, which is consistent with what has happened since 2007 in commercial airplane industry.
\end{abstract}


In addition, the case study of the Exascale supercomputer development is presented to demonstrate the predictive use of the new method. The results indicate that the current development target of 2020 might entail technical risks considering the rate of change emphasizing power efficiency observed in the past. It is forecasted that either a Cray-built hybrid system using Intel processors or an IBM-built Blue Gene architecture system using PowerPC processors will likely achieve the goal between early 2021 and late 2022 . This indicates that the challenge to improve the power efficiency by a factor of 23 would require the maximum delay of 4 years to reach the Exascale supercomputer compared to the existing performance curve. 


\section{Table of Contents}

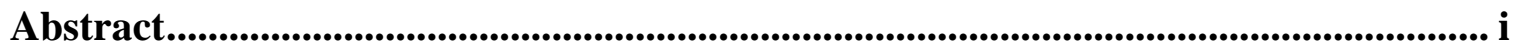

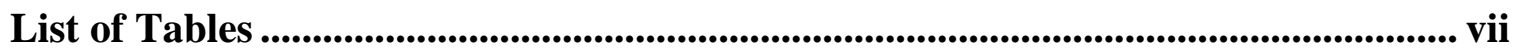

List of Figures......................................................................................................................... viii

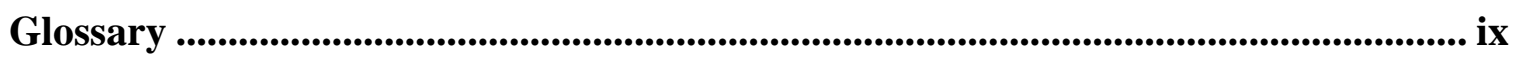

List of Symbols ........................................................................................................................ xi

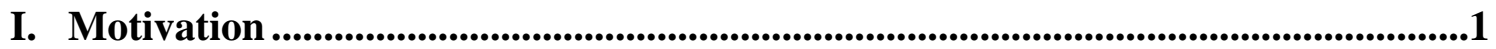

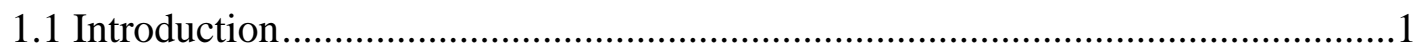

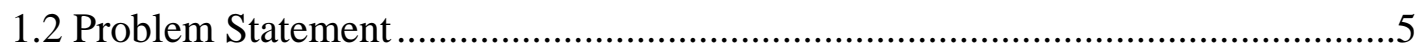

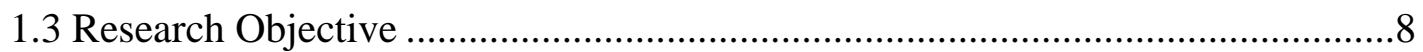

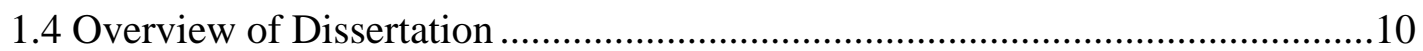

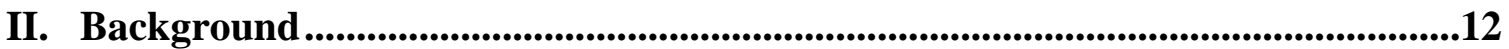

2.1 Technological Innovation Theories ……………………….........................12

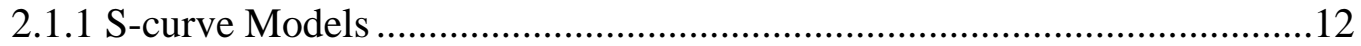

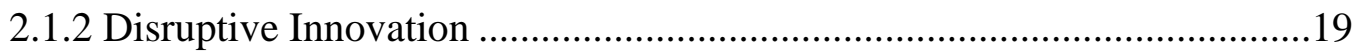

2.1.3 New Product Target Setting..................................................................26

2.2 Technology Assessment Methods......................................................................30

2.2.1 Qualitative Models.................................................................................

A. Delphi and Expert Opinion ...............................................................30

B. Scenario Analysis ..................................................................................

C. Risk Assessment and Environmental Analysis .......................................31

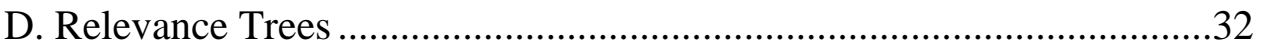

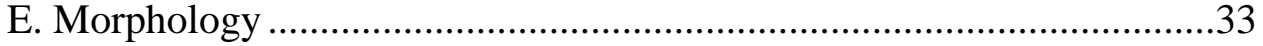

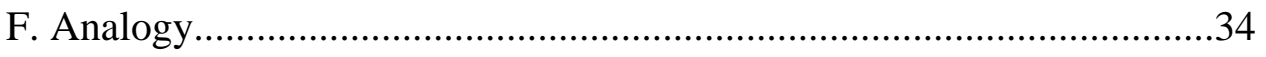

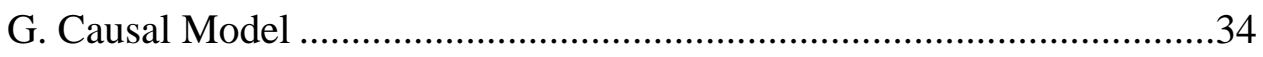

2.2.2 Quantitative Models..................................................................................

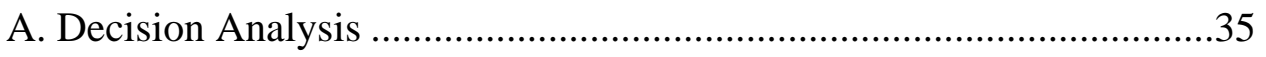

iii 
B. Economic (Cost-Benefit)Analysis............................................................36

C. Modeling (Simulation and System Dynamics) .......................................36

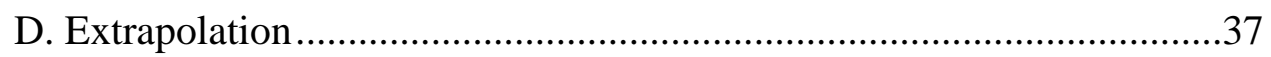

2.3 Focused Review on Multi-Attribute Extrapolation Methods.................................39

2.3.1 Intuitive Models ......................................................................................

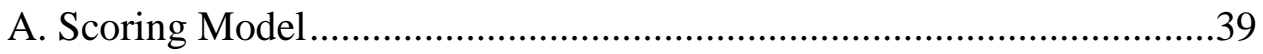

B. Technology Development Envelope ………………………………......40

2.3.2 Parametric Frontier Models …………………………………………....... 41

A. Planar Frontier Model (Hyper-plane) ...................................................41

B. Corrected Ordinary Least Squares ……………………………….........43

C. Stochastic Frontier Analysis ...............................................................4

D. Ellipsoid Frontier Model .......................................................................4

E. Multi-Dimensional Growth Model.......................................................47

2.3.3 Non-parametric Frontier Models ................................................................49

A. Data Envelopment Analysis...................................................................49

B. Stochastic (Chance-constrained) Data Envelopment Analysis ..............54

2.4 Summary of Critical Review .........................................................................56

III. Model Development............................................................................................................58

3.1 Conceptual Framework ………………………………..................................58

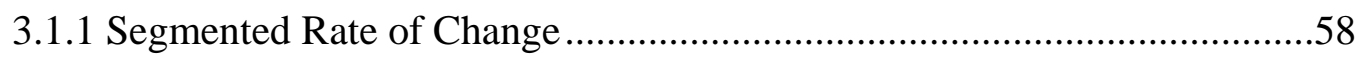

3.1.2 Infeasible Forecasting Target...................................................................64

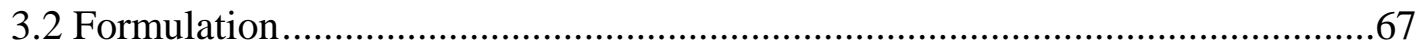

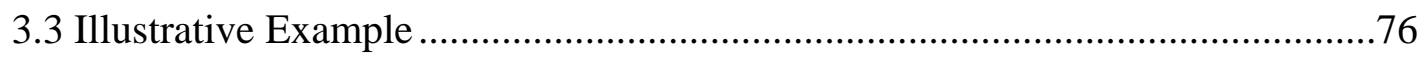

IV. Validating through Case Studies.......................................................................................80

4.1 Ex Post Analysis: Revisit Earlier Studies .........................................................

4.1.1 Risk Analysis: Commercial Airplane Development...................................81

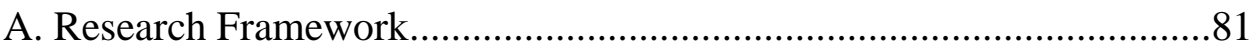

B. Analysis of Current State-of-the-art....................................................83 


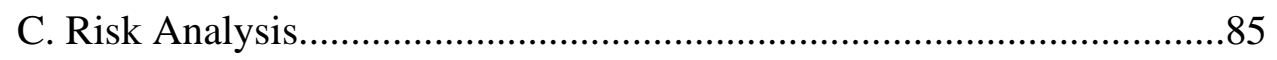

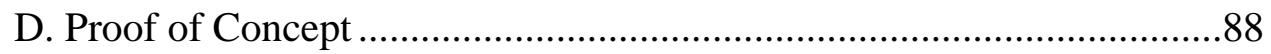

4.1.2 Validation using Past Datasets..............................................................90

A. Forecasting Accuracy Evaluation Techniques .......................................90

B. Test Results from Earlier Studies ..........................................................92

4.2 Ex Ante Analysis: Focused Application.........................................................95

4.2.1 Exascale Supercomputer Development ...................................................95

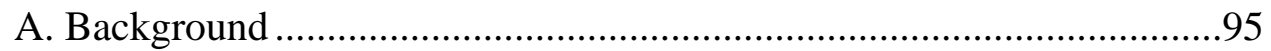

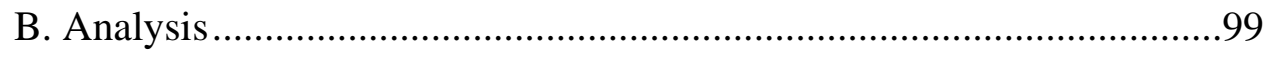

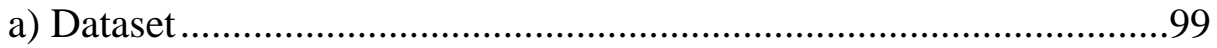

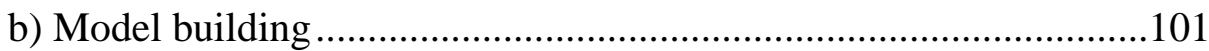

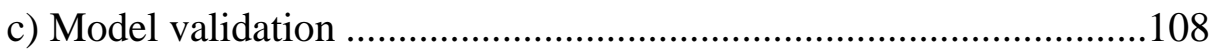

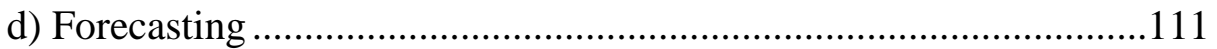

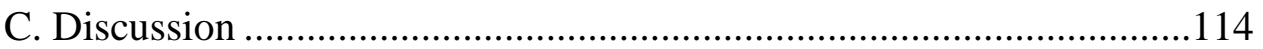

D. Summary of Findings...................................................................118

V. Conclusion ........................................................................................................................120

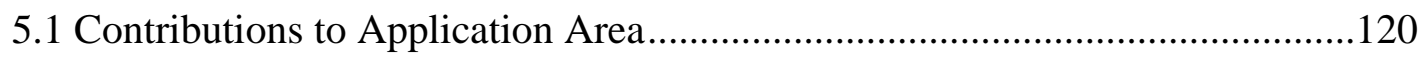

5.1.1 Exascale Supercomputer Development ...................................................120

5.2 Contributions to Managerial Insight ................................................................122

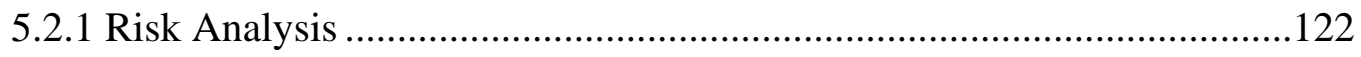

5.2.2 New Product Target Setting ................................................................123

5.3 Contributions to Methodological Development.................................................124

5.3.1 Identification of Local Rate of Change......................................................124

5.3.2 Identification of Individualized Rate of Change......................................124

5.3.3 A Finite Forecast for an Infeasible Target .................................................125

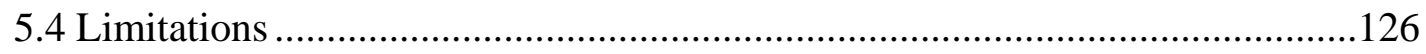

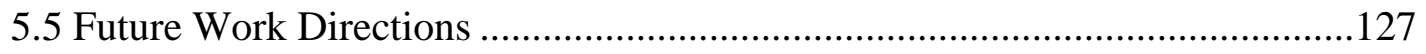

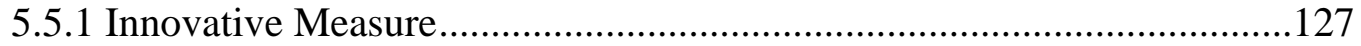

5.5.2 Alternate Efficiency Measures ..................................................................127 


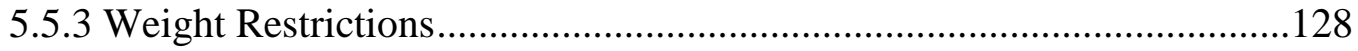

5.5.4 Time Varying Rate of Change ..............................................................129

5.5.5 Direction of Technological Progress ..................................................129

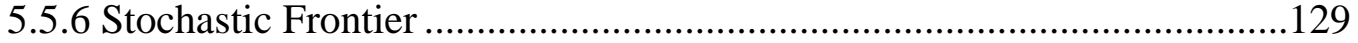

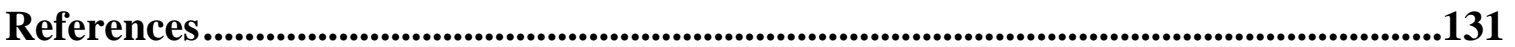

Appendix. Model Building Guideline..........................................................................158 


\section{List of Tables}

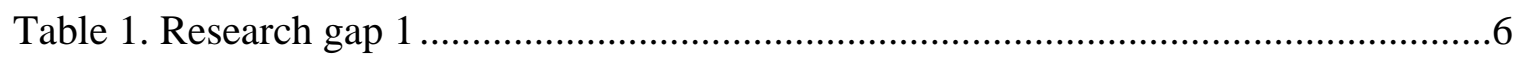

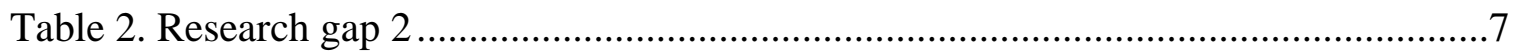

Table 3. Research gap 3 .....................................................................................

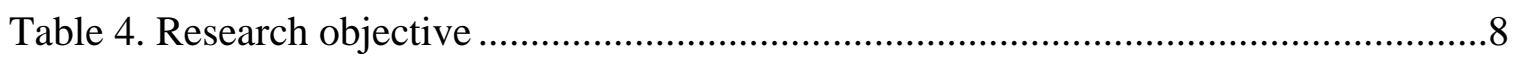

Table 5. Research question 1 ....................................................................................

Table 6. Research question 2 ...........................................................................

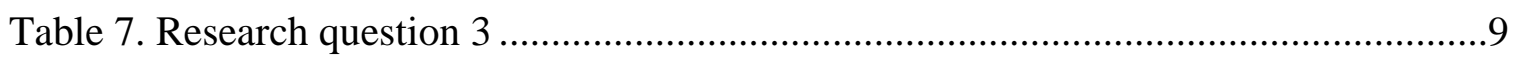

Table 8. Commonly used growth models .....................................................................17

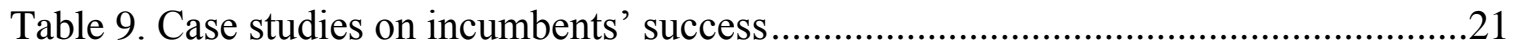

Table 10. Summary of literatures on the technology trajectory mapping.........................25

Table 11. Summary of literatures on the new product target setting .................................29

Table 12. Summary of multi-attribute technology assessment models .............................57

Table 13. Forecast results for infeasible targets............................................................

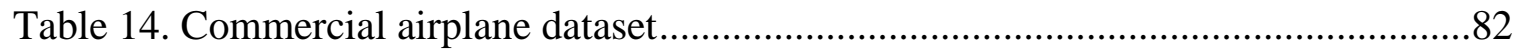

Table 15. Local RoC of SOA airplanes at the frontier year of 2007 .................................82

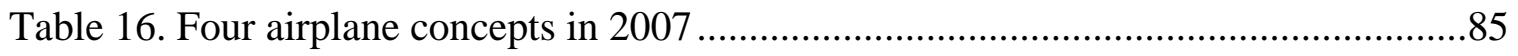

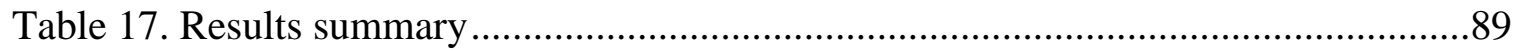

Table 18. Forecast accuracy comparisons ………….....................................................92

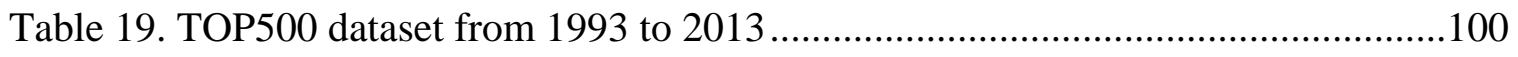

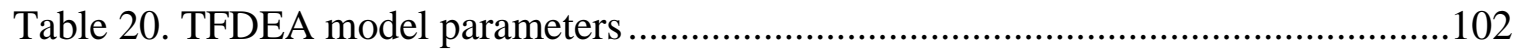

Table 21. Specifications of 13 SOA supercomputers …………......................................103

Table 22. Model validation using a rolling origin hold-out sample tests ........................109

Table 23. Exascale computer as a forecasting target ..........................................................111

Table 24. Forecast results of Exascale supercomputer .....................................................113

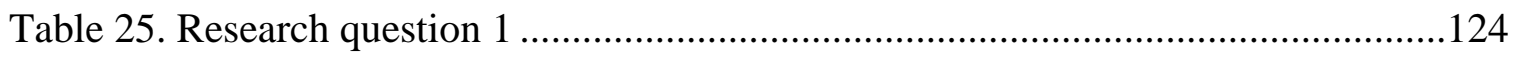

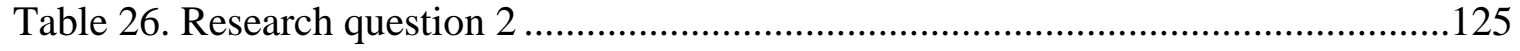

Table 27. Research question 3 .......................................................................125

Table 28. Alternate non-radial efficiency measures .......................................................128 


\section{List of Figures}

Figure 1. Complexity of technology assessment ...................................................

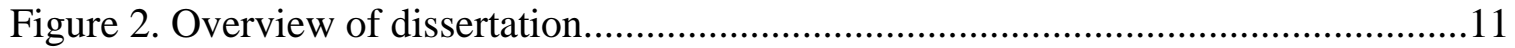

Figure 3. Ambiguity of using time as a proxy for engineering effort ............................15

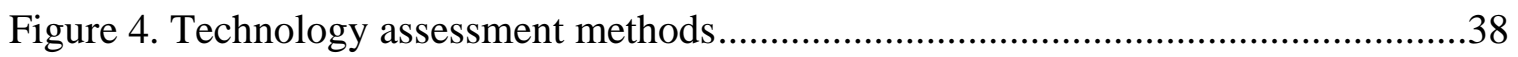

Figure 5. Frontier models in input-output space .......................................................44

Figure 6. Two-dimensional illustration of ellipsoid frontier .......................................46

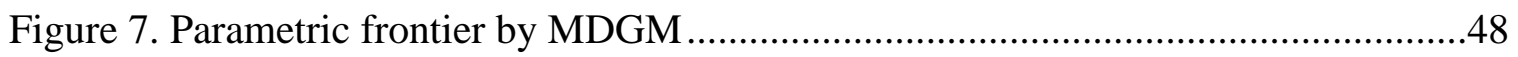

Figure 8. Non-parametric frontier by TFDEA .......................................................52

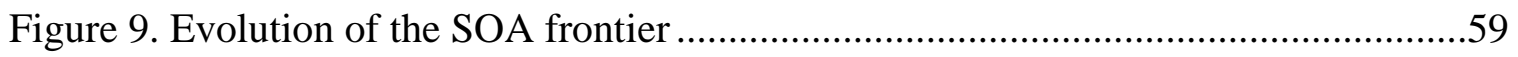

Figure 10. Illustration of segmented rate of change ................................................62

Figure 11. Regions of infeasible super-efficiency under VRS ..................................65

Figure 12. Forecast deviation distributions ........................................................... 78

Figure 13. 2007 state-of-the-art frontier with regard to four design concepts ..................87

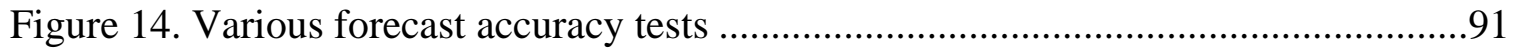

Figure 15. Relative comparison of segmented RoC with constant RoC.........................94

Figure 16. System tradeoffs to be considered for the future HPC trend .........................97

Figure 17. 13 State of the art supercomputers considering system tradeoffs .................102

Figure 18. Performance trajectories of different processor families.............................105

Figure 19. Individualized RoCs with respect to the local RoCs .................................112 


\section{Glossary}

\begin{tabular}{|c|c|}
\hline Abbreviation & Description \\
\hline AHP & Analytical Hierarchy Process \\
\hline AMD & Advanced Micro Devices \\
\hline $\mathrm{C} 3$ & Cryogenic Computer Complexity \\
\hline CAPM & Capital Asset Pricing Model \\
\hline CI & Confidence Interval \\
\hline $\mathrm{CM}$ & Customer Matrix \\
\hline COLS & Corrected Ordinary Least Square \\
\hline $\mathrm{CPU}$ & Central Processing Unit \\
\hline CRA & Comparative Risk Assessment \\
\hline CRS & Constant Returns to Scale \\
\hline DEA & Data Envelopment Analysis \\
\hline DMU & Decision Making Unit \\
\hline DOE & Department of Energy (U.S.) \\
\hline DRS & Decreasing Returns to Scale \\
\hline DVD & Digital Video Disk \\
\hline EIA & Environmental Impact Analysis \\
\hline EIS & Entry Into Service \\
\hline FCM & Fuzzy Cognitive Map \\
\hline FLOPS & FLoating-point Operations Per Second \\
\hline FMEA & Failure Mode and Effects Analysis \\
\hline FS & Frontier Shift \\
\hline GDF & Geometric Distance Function Model \\
\hline GPU & Graphics Processing Unit \\
\hline HDM & Hierarchical Decision Model \\
\hline HPC & High Performance Computing \\
\hline IARPA & Intelligence Advanced Research Projects Activity \\
\hline IO & Input-Oriented \\
\hline IRS & Increasing Returns to Scale \\
\hline LCC & Life Cycle Cost \\
\hline LCD & Liquid Crystal Displays \\
\hline
\end{tabular}




\begin{tabular}{|c|c|}
\hline $\operatorname{MCDA}(\mathrm{M})$ & Multi-Criteria Decision Analysis(Method) \\
\hline MDGM & Multi-Dimensional Growth Model \\
\hline MPI & Malmquist Productivity Index \\
\hline MW & Mega-Watt \\
\hline NDRS & Non-Decreasing Returns to Scale \\
\hline NEC & Nippon Electric Company \\
\hline NFC & Near Field Communication \\
\hline NIRS & Non-Increasing Returns to Scale \\
\hline NNSA & National Nuclear Security Administration (U.S.) \\
\hline NSF & National Science Foundation \\
\hline NUDT & National University of Defense Technology \\
\hline OEM & Original Equipment Manufacturer \\
\hline OLED & Organic Light Emitting Diodes \\
\hline $\mathrm{OO}$ & Output-Oriented \\
\hline PFE & Passenger Fuel Efficiency \\
\hline PPS & Production Possibility Set \\
\hline P-SBM & Proportional Slack-Based Model \\
\hline RAICS & RIKEN Advanced Institute for Computational Science \\
\hline RAM & Range-Adjusted Model \\
\hline $\mathrm{RCA}$ & Revealed Comparative Advantage \\
\hline Rmax & Maximal LINPACK performance achieved in Gigaflops \\
\hline RMSE & Root Mean Square Error \\
\hline $\mathrm{RoC}$ & Rate of Change \\
\hline RTS & Returns To Scale \\
\hline SBM & Slack-Based Model \\
\hline SDEA & Stochastic Data Envelopment Analysis \\
\hline SFA & Stochastic Frontier Analysis \\
\hline SOA & State Of the Art \\
\hline TDE & Technology Development Envelope \\
\hline TEC & Technical Efficiency Change \\
\hline TFDEA & Technology Forecasting using Data Envelopment Analysis \\
\hline UHD & Ultra-High Definition \\
\hline VRS & Variable Returns to Scale \\
\hline W-LAN & Wireless Local Area Network \\
\hline
\end{tabular}




\section{List of Symbols}

\begin{tabular}{|c|c|}
\hline Symbol & Description \\
\hline$j(k)$ & Each product/technology being compared to (being evaluated) \\
\hline$i$ & Input variable index \\
\hline$r$ & Output variable index \\
\hline$m$ & The number of input variables \\
\hline$s$ & The number of output variables \\
\hline$T$ & Time of current frontier \\
\hline$t_{j}$ & Time of release of product $j$ \\
\hline$x_{i j}$ & Input $i$ of product $j$ \\
\hline$y_{r j}$ & Output $r$ of product $j$ \\
\hline$\phi_{k}^{R}$ & Output-oriented efficiency of product $k$ at the time of release \\
\hline$\phi_{k}^{C}$ & Output-oriented efficiency of product $k$ at current frontier \\
\hline$\theta_{k}^{R}$ & Input-oriented efficiency of for product $k$ at the time of release \\
\hline$\theta_{k}^{C}$ & Input-oriented efficiency of product $k$ at current frontier \\
\hline$\lambda_{j k}^{R}$ & $\begin{array}{l}\text { Output-oriented intensity vector of product } k \text { citing product } j \text { at the time of } \\
\text { release }\end{array}$ \\
\hline$\lambda_{j k}^{C}$ & $\begin{array}{l}\text { Output-oriented intensity vector of product } k \text { citing product } j \text { at current } \\
\text { frontier }\end{array}$ \\
\hline$\mu_{j k}^{R}$ & $\begin{array}{l}\text { Input-oriented intensity vector of product } k \text { citing product } j \text { at the time of } \\
\text { release }\end{array}$ \\
\hline$\mu_{j k}^{C}$ & $\begin{array}{l}\text { Input-oriented intensity vector of product } k \text { citing product } j \text { at current } \\
\text { frontier }\end{array}$ \\
\hline$\gamma_{k}^{C}$ & Output-oriented rate of change of product $k$ at current frontier \\
\hline$\delta_{j}^{C}$ & Output-oriented local rate of change of product $j$ at current frontier \\
\hline$\beta_{k}^{C}$ & Input-oriented rate of change of product $k$ at current frontier \\
\hline$\zeta_{j}^{C}$ & Input-oriented local rate of change of product $j$ at current frontier \\
\hline$\chi_{k}$ & Output-oriented radial super-efficiency of product $k$ \\
\hline$\psi_{k}$ & Output-oriented radial extremity of product $k$ \\
\hline$\tau_{k}$ & Input-oriented radial super-efficiency of product $k$ \\
\hline$\rho_{k}$ & Input-oriented radial extremity of product $k$ \\
\hline$t_{k}^{\text {forecast_oo }}$ & Output-oriented forecasted time of arrival of product $k$ \\
\hline$t_{k}^{\text {forecast_IO }}$ & Input-oriented forecasted time of arrival of product $k$ \\
\hline
\end{tabular}




\section{MOTIVATION}

\subsection{INTRODUCTION}

If the future unfolded as foretold, individuals and governments would get a great benefit from their actions taken in advance. In addition to handsome payoffs from Wall Street, semiconductor companies could perfectly meet their market demands with newly built fabs, and sportswear companies could get the maximum advertising effects by grasping rising sports stars with long-term contracts. However, in practice, black swan events often render our plans just plain useless or ineffective [1], [2]. Hence, the choice between alternative pathways under estimated future states may significantly alter competitive performance. Note that decision making is inseparable from how the future is expressed. Indeed, we explicitly or implicitly pay attention to the trends and ideas that are shaping the future which form the basis of our everyday decisions.

Future research community theoretically differentiates prediction from forecasting. A prediction is concerned with the future that is preordained and no amount of action in the present can influence the outcomes. Therefore, it is an apodictic, i.e., non-probabilistic, statement on an absolute confidence level about the future [3]. Clearly, the goodness of a prediction lies in whether it eventually comes true. A forecast, on the other hand, is a probabilistic statement on a relatively broad confidence level about the future. Fundamentally, it aims to affect the decision making process by investigating possible signals related to the future events using systematic logic that forecasters must be able to articulate and defend [4]. Thus, except for particular purposes (e.g., benchmark study), a good forecast is determined not by whether it eventually came true but by whether it 
could provide reasonable grounds to support an organization's actions to anticipate identified uncertainties, thereby resulting in a better future than what was most likely without specific actions. This is consistent with a popular saying in Oriental philosophy: "The ultimate goal of forecasting is to make that forecast wrong."

Just as in other areas of forecasting, technological forecasting intends to improve the quality of decisions by providing specific pieces of information focused on technologies. Lenz identified six major roles of technological forecasting that can be summarized as follows [5]:

1. To identify limits of current technology systems

2. To establish rates of progress

3. To describe technology alternatives

4. To indicate the feasibilities of technology alternatives

5. To provide a reference standard for the new product development plan

6. To furnish warning signals

Technological forecasting methods can be classified as either exploratory or normative by whether they extend present trends (exploratory) or look backward from a desired future to determine the developments needed to achieve it (normative) [6]. The correct assessment of the future environment and of the corresponding goals, requirements, and human desires can be better made when exploratory and normative components are joined in an iterative feedback cycle [3]. Here, it is crucial to have an accurate understanding of the technological inertia we have today so that exploratory methods extend the current rate of progress, while normative methods determine how 
much the speed of such progress might need to be adjusted. However, as technology systems become more sophisticated, the rate of change varies more dramatically due to the maturity levels of component technologies [7]. This structural complexity makes today's forecasting even more challenging, which leads to the question: What is the best way to combine growth patterns of the various attributes used to describe multi-objective technology systems?

To answer the above question, two things must be considered: multi-attribute evaluation and technology segmentation. Multi-attribute evaluation strives to define the "goodness" of technology systems that consist of different levels of subsystems. Figure 1 illustrates the difficulty of doing this. Technology B seems to have made a disruption in a high-end market, while technology $\mathrm{A}$ is overshooting the market in terms of technical capacity 1 . However, technology 2 might have been superior and recently challenged by technology 1 on a different technical dimension. Possibly, different dynamics are taking place in other dimensions as well where the levels of market demands also vary. This implies that a single performance measure may be no longer capable of capturing advancement in a new direction, which makes the holistic assessment of technology systems difficult. Therefore, it is critical to examine not only which performance measures are playing a major role in current technological progress but also which alternate technologies show disruptive potential with respect to emerging performance measures.

Technology segmentation is related to the identification of homogeneous technology clusters. Technologies belonging to the same cluster may have a similar mix of technical capabilities whereby they satisfy the similar target markets. From the technological 
forecasting point of view, each technology cluster is expected to involve a particular progress pattern to which similar types of future technologies are subjected. Thus, it is imperative for a forecasting model to be able to identify distinct technological segments, if they exist, and treat them separately in the process of capturing the rate of change and estimating the future performance specifications and features.

This dissertation aims to develop a new frontier analysis method for technological forecasting that can deal with foregoing two issues: multi-attribute evaluation and technology segmentation.

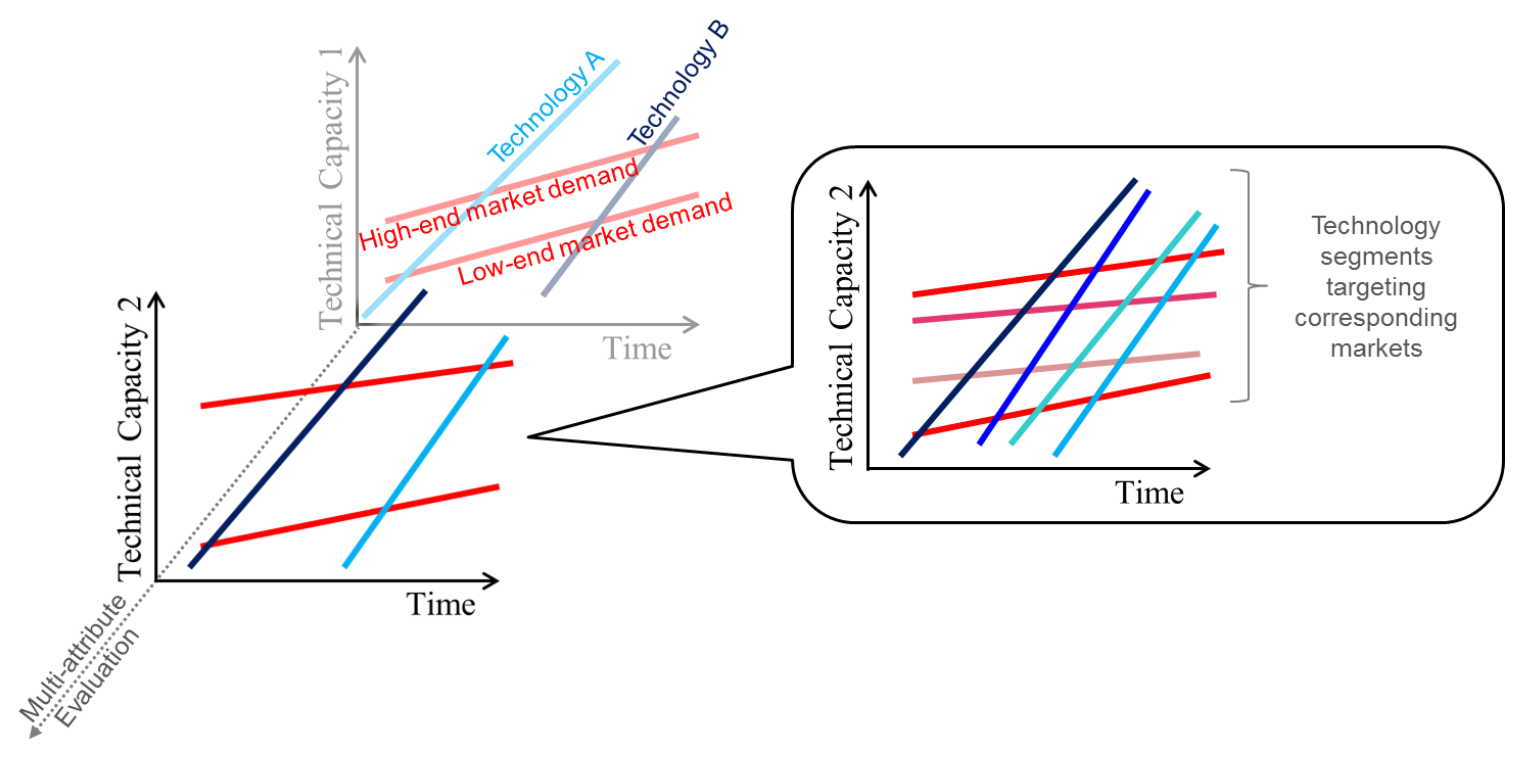

Figure 1 Complexity of technology assessment 


\subsection{Problem Statement}

Technology growth curve (or life cycle) theory asserts that the accumulated performance of a technology forms an S-shaped curve when it is plotted over time, and the diminishing returns to effort is successively overcome by following growth curves that possess higher performance limits [8]-[11]. Although S-shaped growth patterns have been observed in numerous studies [12]-[14], a fact well known is that fitting some portion of cumulative data to a predefined S-curve function, i.e., predictive use of the curve, is susceptible to several technical assumptions such as engineering effort, is constant over time, and an upper limit is known in advance.

Disruptive innovation theory integrates the technological growth patterns with different market acceptance levels to explain the process of new value network creation [15]. Even though disruptive innovation has contributed to the understanding of industry dynamics, the practical implications remain debatable [16]-[18]. In particular, the theory is criticized that it lacks the mechanism for the predictive use [19]. Christensen suggested a diagram that jointly portrays trajectories over time of performance demanded by different market segments and of performance provided by alternative technologies [20]. However, this comes down to the difficulties of employing a growth curve model as an ex ante analysis.

The recent development of product categorization methods can be viewed as another stream of literature with regard to the topic of risk analysis for new product development [21]-[25]. However, although these approaches can shed light on the new product target setting practices, there remains a need to integrate the product benchmarking with the 
assessment of performance improvement so that analysts can have a risk measure for their product launch strategy.

The above mentioned problems can be summarized as below.

Table 1 Research gap 1

GAP

$\# 1$

Current technological innovation and new product development theories do not provide a quantitative framework to facilitate the predictive use of the theory.

Frontier analysis models attempt to form a surface that can represent the same level of technology systems at given point in time. The evolution of surfaces is then monitored to capture the rate of change by which future technological possibilities can be estimated.

In the case of parametric frontier methods, an iso-time frontier is constructed as a functional combination from individual growth curves. Specifically, actual observations are fitted to an a priori defined functional form, and those growth patterns are combined together to constitute an iso-time frontier. Therefore, it is difficult to identify distinct technological segments from the resulting frontier.

Non-parametric frontier methods, on the other hand, have an advantage with regard to the technology segmentation since the frontier is directly constructed by dominating technologies that are located on the frontier. This enables the model not only to characterize each frontier segment but also to identify proper segments that dominated technologies belong to. However, current non-parametric frontier models don't incorporate this property into the forecasting process. Instead, they simply aggregate rate of changes captured from the surpassed technologies to indicate the technological progress as a whole. 
The above mentioned issues can be summarized as below.

Table 2 Research gap 2

GAP

\#2

Current technological forecasting methods do not take into account technology segmentation.

Non-parametric frontier methods reflect the distinct characteristics of the technology systems by directly adapting to observed data without relying on arbitrary functional assumptions [26]. However, this characteristic often leads to an infeasibility problem since it doesn't generate the frontier facet for non-existent production possibilities in the past [27]. This becomes a critical problem when the model estimates the distance from the current frontier to forecasting targets. In other words, the model may fail to measure the goodness of future technologies if it has an unprecedented mix of technical capabilities. This issue can be summarized as below.

Table 3 Research gap 3

Current non-parametric frontier analysis methods often suffer from concerns regarding infeasibility. 


\subsection{ReSEARCH ObJECTIVE}

Based on the aforementioned research gaps, the objective of this research is specified as below.

Table 4 Research objective

Research Objective
Develop a new frontier analysis method for technological forecasting that is capable of multi-attribute evaluation by considering technology segmentations with the corresponding rate of changes.

The technology segmentation process can be understood as two procedural stages.

First, it is required for the model to identify distinct facets on the frontier in which local rates of change can be obtained from the surpassed technologies. By doing so, each state-of-the-art technology will have a local rate of change which indicates how much progress has been observed by each technological segment represented by those technologies. This corresponds to the first research question as below.

Table 5 Research question 1

Research

Question

\#1
How do we capture the local rate of change from past technologies?

Once the local rates of change are ascertained with respect to each frontier segment, they can be utilized to obtain the individualized rate of change for each forecasting target. This procedure makes it possible for the model to apply the customized progress rate suitable for each forecasting target, thereby reflecting the characteristics of identified segments into the forecast. This leads to the second research question as below. 
Table 6 Research question 2

Research

Question

\#2

How do we determine the individualized rate of change for future technologies?

As previously discussed, non-parametric features of identifying the technology segmentations come at a cost to the infeasibility problem. Therefore, a new model should be able to provide an alternative forecasting mechanism in a consistent manner, i.e., without violating a radial distance measure, for the case when the infeasibility problem occurs. This requires an assumption that the superiority of unprecedented types of technologies can be estimated by the consideration of how far the target technology is away from the closest technology segment as well as how good the target technology is from a perspective of the closest technological segment. The formulation of this procedure is subject to the following research question.

Table 7 Research question 3

\section{Research}

Question

How do we deal with infeasible targets? 


\subsection{OVERVIEW OF DisSERTATION}

This dissertation is organized as follows. In Chapter II, critical literature reviews on technological innovation theories and technological forecasting methodologies are provided to address the objective of this dissertation. In Chapter III, the notion of segmented rate of change is illustrated using a numerical example as well as mathematical formulation to supply insight into the problem being discussed. In Chapter IV, a set of applications is provided to demonstrate and validate the proposed model. Finally, Chapter V summarizes contributions and suggests possible future research directions. 


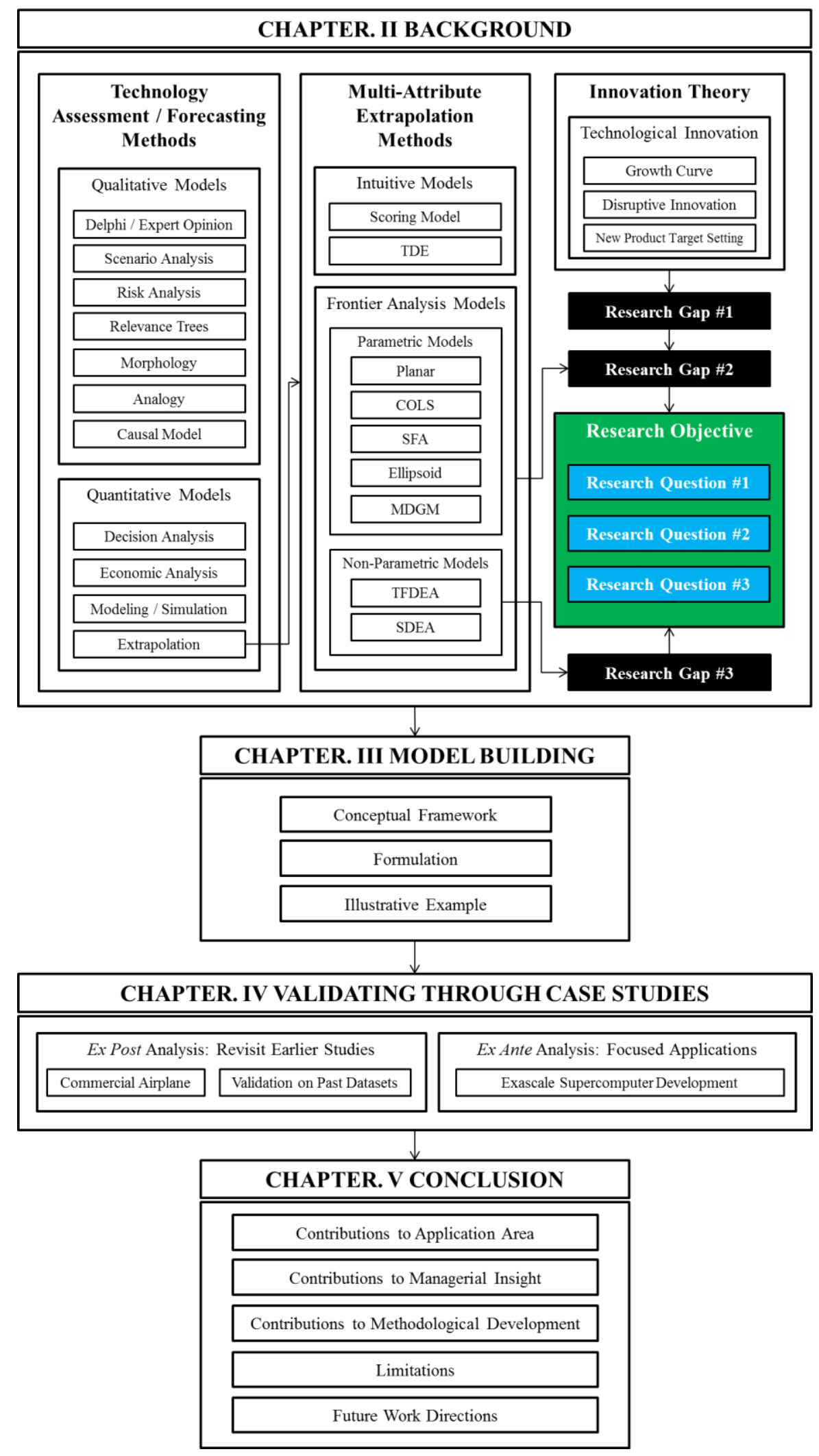

Figure 2 Overview of dissertation 


\section{BACKGROUND}

\subsection{TECHNOLOGICAL INNOVATION THEORIES}

\subsubsection{S-CURVE MODELS}

Beginning with Kondratiev's early observation in 1925, technological innovation has been believed to exhibit a wave-like phenomenon that consists of life cycles of individual technologies [8]-[11]. According to this view, the accumulated performance of a technology forms an S-shaped curve when it is plotted over time, and the diminishing returns to effort is successively overcome by following growth curves that possess higher performance limits. In this belief, Cesare Marchetti once claimed that "Anything that begins and ends an existence will fit a logistic (also known as a Pearl curve)" during the conversation with Theodore Modis, who holds the Guinness Book of Records for carrying out the greatest number of logistic fits [28].

Simply speaking, an S-curve is a natural outcome which is a cumulative function of growth levels from typical evolution stages: introduction stage (slow acceleration), growth stage (speed up), and maturity stage (deceleration). Therefore, it may be worth understanding the reason why the rates of progress in each stage are different and especially what hinders the growth during introduction and maturity stages.

In the introduction (or embryonic) stage, a new technological platform makes slow progress, mostly due to the insufficient interests of the research community [29]. A consensus between alternate ways of overcoming the bottlenecks is called for; however, there may be no measures for assessing the new technology properly, which makes the circumstances of the decision be based less on problem solving ability than on future 
promise [30]. Therefore, until the new approach has gained established legitimacy as a worthwhile endeavor, great effort is often spent exploring different paths to identify meaningful and feasible drivers of advancement. For example, OLED (organic light emitting diodes) technology has been recently introduced as a new alternative to LCD (liquid crystal displays) in the flat panel industry. However, it requires a sufficient amount of time and effort to identify the direction of incremental innovation.

In the growth stage, a new technological platform finally crosses a threshold with continuous engineering effort, which allows rapid progress [13]. The emergence of a dominant design, in particular, plays a key role not only to attract researchers to participate in its development but also to coalesce product characteristics and consumer preferences [12]. The cumulative efforts reap the greatest improvement per unit of effort, which creates a virtuous cycle by stimulating more attention devoted to the current technological platform.

In the maturity stage, the progress slowly and asymptotically reaches a ceiling [10]. Utterback suggested that as a market ages, the focus of innovation shifts from product to process innovation [31]. Sahal also argued that technology has inherent limits of scale and/or complexity which restrict the steady growth of performance improvement [14]. As such, a marginal performance increase requires more cost and engineering efforts, which eventually deglamorize the current technological platform. As the current technological platform loses its luster, the research community searches for alternative paths and rapidly loses cohesion, which reduces the switching costs to the upcoming technology.

Although the S-shaped growth pattern has been observed in a number of studies that conducted retrospective analysis on industry dynamics, it is well known that fitting some 
portion of cumulative data into a predefined S-curve function is susceptible to several technical assumptions, which renders the predictive use of the curve ambiguous [32].

The first assumption is that the engineering effort is constant over time. Fundamentally, the S-curve reflects the level of technological capability relative to the cumulative effort invested in developing the technology [33]. However, engineering effort is rarely used as an abscissa in practical applications due to the difficulty in tracking the cumulative effort over an entire technology cycle. Instead, there are two alternative parameters that are often used in place of engineering effort: research and development investment or time. However, the problem arises when these proxy measures are not proportional to actual engineering efforts. Fig. 3 shows that the resulting curve can obscure the true relationship, which could appear to flatten out much more quickly, or not to flatten out at all when this assumption is not supported [34]. 

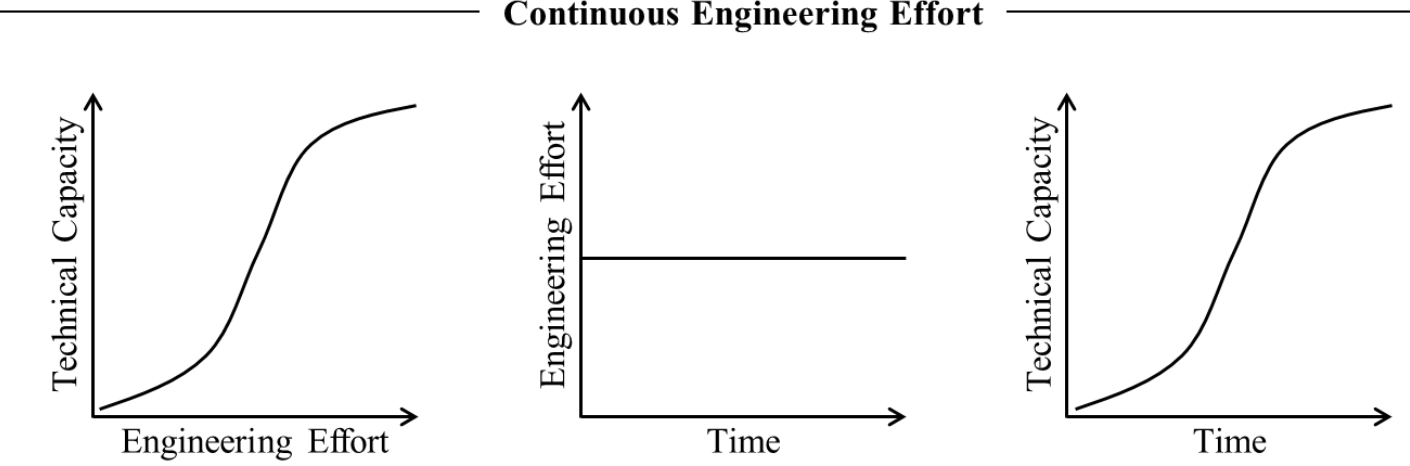

Engineering effort can be replaced by time to obtain the same curve

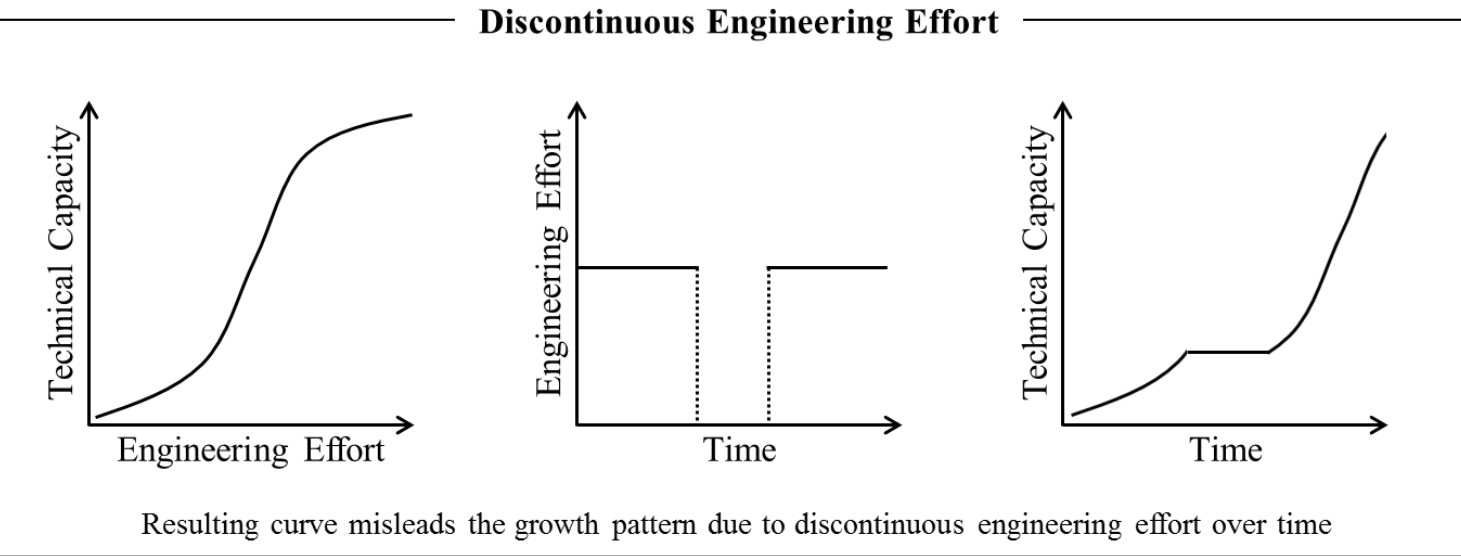

Figure 3 Ambiguity of using time as a proxy for engineering effort (modified from [32])

The second assumption is that the upper limit of a growth curve, i.e., $L$, is given. However, it is rare that the true limit of a technology is known in advance, and there is often considerable disagreement about what the limits of a technology are. A well-known case of misperception can be found in the disk drive industry [35]. In 1979, IBM had reached what it perceived as a density limit of ferrite-oxide-based disk drives; therefore, the company moved to developing thin-film technology that had a greater potential for increasing density. Hitachi and Fujitsu, however, continued to ride the ferrite-oxide S- 
curve, and ultimately achieved densities that were eight times greater than the density that IBM had perceived to be a limit.

Due to the lack of information, researchers often have no choice but to employ regression-based calculation to estimate the upper limits of technology growth curves [6]. However, this approach is controversial in the literature [36], [37]. Danner's simulation showed that the accuracy of the resulting limit is highly sensitive to any error present in the segment of the available data [32]. Martino also argued that the productivity of early technology development is only minimally influenced by the upper limit because historical data from the early stages of development contain little information as to the location of the upper limit [38]. In this sense, he claimed that even a small error in the upper limit estimation can result in a fairly significant error in the forecast.

The third assumption is that the appropriate growth model is predefined. However, similar to the estimation of upper limits, a growth curve should not be selected based on goodness of fit from historic data but on matching the behavior of the selected growth curve to the underlying dynamics of technology growth [39], [40]. In fact, there are various equations that represent $\mathrm{S}$-shaped curves which can be categorized into two main groups: absolute and relative models. The former quantifies the technical capability, $y_{t}$, as a function of the independent parameter time, $t$, whereas the latter quantifies the rate of change in technical capability, $d y_{t}$, as a function of the most recently achieved level of technical capability, $y_{t-1}$ (see Table 8) [32], [41].

Young's study showed that relative models were more accurate than absolute models, and in particular both the Bass and Harvey growth models performed well under most circumstances [37]. Danner claims that this may be because the inherent characteristic of 
the relative model that each new data point is anchored to the previous data point. That is, changes in the relative model are proportional to both the progress to date and the distance to the upper limit, whereas changes in the absolute model are only proportional to the distance from the upper limit [32].

Table 8 Commonly used growth models [32], [37]

\begin{tabular}{lll}
\hline Type & Name & Equation \\
\hline Absolute & Logistic (Pearl) [42] & $y_{t}=\frac{L}{1+\alpha e^{-b t}}$ \\
\cline { 2 - 3 } & Gompertz [43] & $y_{t}=L e^{-\alpha e^{-b t}}$ \\
\cline { 2 - 3 } & Linear Gompertz [41] & $\ln \left(-\ln \left(\frac{y_{t}}{L}\right)\right)=\beta_{0}+\beta_{1} t$ \\
\cline { 2 - 3 } & Mansfield-Blackman [44] & $\ln \left(\frac{y_{t}}{L-y_{t}}\right)=\beta_{0}+\beta_{1} t$ \\
\cline { 2 - 3 } & Weibull [45] & $\ln \left(\ln \mid \frac{y_{t}}{L-y_{t}}\right)=\beta_{0}+\beta_{1} \ln t$ \\
\cline { 2 - 3 } & Von Bertalanffy [43] & $y_{t}=\left(1-\alpha e^{-b t}\right)^{3}$ \\
\cline { 2 - 3 } & S-curve [43] & $y_{t}=e^{a-(b / t)}$ \\
\hline Relative & Bass [46] & $d y_{t}=\beta_{0}+\beta_{1} y_{t-1}+\beta_{2} y_{t-1}{ }^{2}$ \\
\cline { 2 - 3 } & Non-symmetric Responding & $\ln d y_{t}=\beta_{0}+\beta_{1} \ln \left(y_{t-1}\right)+\beta_{2} \ln \left(L-y_{t-1}\right)$ \\
\hline & Logistic [47] & $\frac{d y_{t}}{y_{t-1}}=\beta_{0}+\beta_{1} y_{t-1}+\beta_{2}\left(\frac{1}{y_{t-1}}\right)+\beta_{3} \ln \left(y_{t-1}\right)$ \\
\hline Harvey [48] & \\
\cline { 2 - 3 } & Extended Riccati [49] & $\ln d y_{t}=\beta_{0}+\beta_{1} t+\beta_{2} \ln \left(y_{t-1}\right)$ \\
\hline
\end{tabular}

However, it should be noted that the underlying assumption of the relative model that future advancement is facilitated by technical capability already achieved may not always be true. In a similar context, Young's finding, based on 46 historical data sets, 
might not guarantee the most appropriate selection for the predictive problem at hand. Therefore, model selection should consider how the model's behavior matches the process that generated the data rather than simply fitting the historical data [50].

The shape of a technology's growth curve is neither set in stone nor given to the analyst. The limitation of the current architecture can be overcome by technological innovation which affects the growth rate and possibly allows a higher performance to be achieved than what had been perceived to be a limit. On the contrary, the lifecycle of a given technology could be terminated by the unexpected adoption of alternative technologies even before it passes the inflection point of the curve [51]. Therefore, fitting a portion of data into an a priori defined growth function should be accompanied by a deep understanding of the dynamics of the industry being investigated. 


\subsubsection{DISRUPTIVE INNOVATION ${ }^{1}$}

Christensen asserted his theory of disruptive innovation by integrating technological growth patterns with different market acceptance levels to explain the process of new value network creation [15], [52]. The core premises of his theory can be summarized as follows [17], [18]:

a) A new technology consisting of off-the-shelf components put together in product architecture provides different values from mainstream technologies and initially underperforms the dominant one;

b) Products based on disruptive technologies could, therefore, only serve niche segments without attracting attention of the leading firms;

c) Disruptive technologies steadily improve in performance until it meets the standards of performance demanded by the mainstream market;

d) Further development could raise the disruptive technology's performance to a level sufficient to satisfy mainstream customers, while the established technology could have exceeded the demand of mainstream customers, resulting in performance overshoot.

Although Christensen's work on disruptive innovation has contributed to the understanding of industry dynamics, the mechanism and practical implications of his theory remain controversial.

First of all, the theory lacks a clear-cut definition to determine whether or not a given technology is considered to be disruptive. Christensen explained that "disruptiveness" depends on whether it is consistent with their business model. For example, the Internet is

\footnotetext{
${ }^{1}$ This section is adapted from a paper accepted in $R \& D$ Management [312]
} 
sustaining to catalog retailers and discount brokers, but it is disruptive to department stores and full-service brokers [20]. Further he described the characteristics of disruptive innovation as being typically simpler, cheaper, and more reliable and convenient than the established one [53]. However, Danneels rebutted that these characteristics may be typical but not necessary characteristics of disruptive technology. He gave examples of Amazon.com, digital cameras, and digital video disk (DVD) that have had higher performance when they were introduced in the mainstream market [16]. Chesbrough also noted that Christensen's studies used inconsistent terminology; in other words, they lacked common criteria to classify different types of technologies [54]. This ambiguous definition also raised a question: What determines whether incumbents fail or succeed in the face of disruptive technology? (see Table 9).

In addition, the predictive use of disruptive innovation theory has been a controversial issue in managerial practice. Cohan contended in his book The Dilemma of the "Innovator's Dilemma" that Christensen tried to support his theory using cherry-picked examples, i.e., only case studies of disruptive technologies that succeeded [19]. He urged that retrospective analysis is subject to bias, and the real challenge to any theory, especially if it is to be useful managerially, is how it performs predictively. Christensen responded to this by suggesting a diagram that jointly portrays trajectories over time of performance demanded by different market segments and of performance provided by alternative technologies [20]. For ex post case studies, using trajectory maps is fairly straightforward since the key performance dimension that resulted in a disruption has been identified and that data on performance demanded and supplied are available. However, ex ante analysis requires predicting what performance the market will demand 
along various dimensions and what performance levels technologies will be able to supply [16].

Table 9 Case studies on incumbents' success

\begin{tabular}{lll}
\hline Author (Year) & Application area & $\begin{array}{l}\text { Did incumbents } \\
\text { succeed? }\end{array}$ \\
\hline Cooper and Schendel (1976) [55] & Videotape, Teleconferencing, etc. & No \\
Foster (1986) [10] & Video game console & No \\
Tushman and Anderson (1986) [56] & Competence destroying discontinuities & No \\
Henderson and Clark (1990) [57] & Copy machine, Radio receiver, etc. & No \\
Henderson (1993) [58] & Photolithographic alignment equipment & No \\
Utterback (1994) [11] & Electric lamp, QWERTY, etc. & No \\
Christensen and Bower (1996) [52] & Seagate, Control Data & No \\
Chesbrough (1999) [59] & Hard disk drive (in U.S) & No \\
McKendrick et al. (2000) [60] & Disk drive (transition to 5.25-inch) & No \\
Tripsas and Gavetti (2000) [61] & Polaroid & No \\
Helfat and Lieberman (2002) [62] & Canon and Nikon & No \\
Tushman and Anderson (1986) [56] & Competence enhancing discontinuities & Yes \\
Chesbrough (1999) [59] & Hard disk drive (in Japan) & Yes \\
Cohan (2010) [19] & Charles Schwab, Kodak and Fuji & Yes \\
McKendrick et al. (2000) [60] & Disk drive (transition to 8 \& 3.5-inch) & Yes \\
King and Tucci (2002) [63] & Disk drive & Yes \\
Danneels (2004) [16] & Non-fossil fuel powered automobile & Yes \\
Klepper and Simons (2000) [64] & Television sets & Yes \\
Chandy and Tellis (2000) [65] & Office products and consumer durables & Yes \\
Tripsas (1997) [66] & Mergenthaler Linotype & Yes \\
Rothaermel (2001) [67] & Pharmaceutical industry & Yes \\
Darby and Zucker (2001) [68] & Chemistry to biotechnology & Yes \\
\hline & &
\end{tabular}

Trajectory mapping has been employed in a wide range of applications. The most famous application of a trajectory map may be the hard disk drive case from Christensen's original work [35]. He used disk capacity as a performance axis and interpreted the dynamics of industry that smaller disks have replaced bigger ones, improving their capacities over time. Schmidt later extended Christensen's work by classifying the disk drive case as a low-end encroachment that eventually diffused 
upward to the high-end [69]. Martinelli conducted patent analysis in the telecommunication switching industry to discover seven generations of technological advances from the different paradigmatic trajectories [70]. Kassicieh and Rahal also adopted patent publication as a performance measure in search of potential disruptive technologies in therapeutics [71]. Phaal et al. proposed a framework that has been tested by developing more than 25 diverse "emergence maps," analogous to a trajectory map, of historical industrial evolution, building confidence that the framework might be applicable to current and future emergence [72]. Keller and Hüsig analyzed Google's web-based office application to see if it can pose a disruptive threat to incumbent technologies, namely Microsoft's desktop office application [73]. Barberá-Tomás and Consoli tried to identify potential disruptive innovations in the medical industry, especially for artificial discs, by counting the number of granted patents over time [74]. Husig, et al. (2005), conducted one of the rare ex ante analyses that mapped out trajectories of both the incumbent technology and a potential disruptive technology [75]. They made a forecast based on a trajectory map that wireless local area network (W-LAN) technologies would not be disruptive for incumbent mobile communications network operators in Germany. This is because the average growth rate of the bandwidth supplied by W-LAN had been overshooting the average growth rate of the bandwidth requirements of all customer groups.

There are a few studies that used composite performance measures to draw the technology trajectories. Adamson plotted $R^{2}$ values from the multiple regression analysis on the trajectory map to investigate the fuel cell vehicle industry [76]. The results showed that the subcompact vehicles' $R^{2}$ values were increasing over time while compact 
vehicles' were decreasing. The author used this information and identified key utility attributes that could command a significant premium before the product reaches the mass market. This study has significant implications for identifying key drivers of technology progress using the trajectory map. Letchumanan and Kodama mapped out the correlation between Revealed Comparative Advantage (RCA), which is generally used to measure the export competitiveness of a product from a particular country in terms of world market share, and R\&D intensity to examine who was making the most disruptive advancement at a national level [77]. Even though Koh and Magee didn't utilize any function to develop composite performance measures, their research has a significance as they took different trade-offs into consideration to draw a trajectory map [78]. Their results suggested that some new information transformation embodiment such as a quantum or optical computing might continue the trends given the fact that information transformation technologies have shown a steady progress.

Few researchers have proposed the predictive approach of the disruptive innovation theory considering multidimensional aspects of technology systems. Schmidt suggested using part-worth curves in search of low-end encroachment [69]. Paap and Katz provided general guidance for ex ante identification of future disruption drivers [79]. Several authors have suggested using extant methods for technological forecasting to assess potential disruptive technologies [16], [17]. Govindarajan and Kopalle argued that capturing a firm's willingness to cannibalize could be a sign of ex ante prediction of disruptive innovation [80]. Doering and Parayre presented a technology assessment procedure that iterates among searching, scoping, evaluating, and committing [81]. 
Table 10 summarizes 40 studies that have employed the trajectory map to identify disruptive alternatives (technology, product, service, etc.). The majority of the studies adopted a single performance measure to draw the trajectory map. A trajectory map, however, should be able to take multiple perspectives into account not to miss potential disruptive indications. Many ex post case studies have shown that disruptions have occurred from an entirely new type of performance measure that hadn't been considered. Furthermore, it was often observed that the new technology started below the prior one in performance on the primary dimension but was superior on a secondary one [18]. This implies that the current performance measure may no longer be capable of capturing advancement in a new direction. Therefore, it is crucial to examine not only which performance measures are playing a major role in current progress but also which alternate technologies show disruptive potential with respect to the emerging performance measures. 
Table 10 Summary of the literature on the technology trajectory mapping

\begin{tabular}{|c|c|c|c|}
\hline Author (year) & Application area & Performance measure & Plotting method \\
\hline Walsh (2004) & Microsystems & Critical dimension & Growth curve \\
\hline Keller \& Hüsig (2009) & Office application & Number of operations & Data accumulation \\
\hline Martinelli (2012) & Telecommunication & Patent citation & Data accumulation \\
\hline Phaal et al. (2011) & S\&T based industry & Sales & Data accumulation \\
\hline Padgett \& Mulvey (2007) & Brokerage market & Service integration level & Data accumulation \\
\hline X. Huang \& Sošić (2010) & General industry & Capacity \& Price & Data accumulation \\
\hline Kaslow (2004) & Vaccine & Efficacy & Data accumulation \\
\hline Kassicieh \& Rahal (2007) & Therapeutics & Patent publication & Patent mapping \\
\hline Christensen (1997) & Disk drive & Capacity & Data accumulation \\
\hline Schmidt (2011) & Disk drive & Part-worth & Data accumulation \\
\hline Rao et al. (2006) & P2P and VoIP & Data transfer & Data accumulation \\
\hline Bradley (2009) & Medical operation & Noninvasiveness & Data accumulation \\
\hline Lucas \& Goh (2009) & Photography & Price, convenience, etc. & Data accumulation \\
\hline Madjdi \& Hüsig (2011) & W-LAN & Active Hotspot ratio & Data accumulation \\
\hline Husig et al. (2005) & W-LAN & Data rates & Data accumulation \\
\hline Walsh et al. (2005) & Silicon industry & Number of firms & Data accumulation \\
\hline Figueiredo (2010) & Forestry industry & Novelty \& complexity & Data accumulation \\
\hline Caulkins et al. (2011) & General industry & Market connection & Skiba curve \\
\hline Adamson (2005) & Fuel cell vehicle & Utility coefficient values & Data accumulation \\
\hline $\begin{array}{l}\text { Belis-Bergouignan et al. } \\
\text { (2004) }\end{array}$ & Organic compound & $\begin{array}{l}\text { Environmental } \\
\text { performance }\end{array}$ & Data accumulation \\
\hline Но (2011) & General industry & Technology sources & Data accumulation \\
\hline Werfel \& Jaffe (2012) & $\begin{array}{l}\text { Smoking cessation } \\
\text { products }\end{array}$ & Patent & $\begin{array}{l}\text { Reduced form } \\
\text { model }\end{array}$ \\
\hline No \& Park (2010) & Nano-biotechnology & Patent & Data accumulation \\
\hline $\begin{array}{l}\text { Letchumanan \& Kodama } \\
(2000)\end{array}$ & $\begin{array}{l}\text { General industry } \\
\text { (High-tech) }\end{array}$ & $\begin{array}{l}\text { Correlation between } \\
\text { Exports and R\&D intensity }\end{array}$ & Data accumulation \\
\hline $\begin{array}{l}\text { Spanos \& Voudouris } \\
(2009)\end{array}$ & Manufacturing SMEs & AMT & Data accumulation \\
\hline $\begin{array}{l}\text { Frenken \& Leydesdorff } \\
\text { (2000) }\end{array}$ & Civil aircraft & $\begin{array}{l}\text { Diffusion rate } \\
\text { (Entropy statistics) }\end{array}$ & Data accumulation \\
\hline Watanabe et al. (2009) & Printers & Sales and price & Price function \\
\hline Hobo et al. (2006) & $\begin{array}{l}\text { Service oriented } \\
\text { manufacturing } \\
\text { industry }\end{array}$ & $\begin{array}{l}\text { Sales, income, employees, } \\
\text { and productivity }\end{array}$ & Data accumulation \\
\hline Watanabe et al. (2005) & Electrical machinery & Marginal productivity & Data accumulation \\
\hline S.-H. Chen et al. (2012) & Smart grid & Average age & Data accumulation \\
\hline Epicoco (2012) & Semiconductor & Devices per chip & Data accumulation \\
\hline Funk (2005) & Mobile phone & Mobile subscribers & Data accumulation \\
\hline Raven (2006) & Renewable energy & Energy Production & Data accumulation \\
\hline Castellacci (2008) & $\begin{array}{l}\text { Manufacturing and } \\
\text { service industries }\end{array}$ & Labor productivity & Data accumulation \\
\hline Kash \& Rycoft (2000) & Radiation therapy & Capability & Growth curve \\
\hline Arqué-Castells (2012) & General industry & Patent & Poisson model \\
\hline W.-J. Kim et al. (2005) & DRAM & $\begin{array}{l}\text { DRAM shipment and } \\
\text { Memory density }\end{array}$ & Data accumulation \\
\hline C.-Y. Lee et al. (2008) & Home networking & New household/year & Data accumulation \\
\hline Koh \& Magee (2006) & & Megabits & Data accumulation \\
\hline $\begin{array}{l}\text { Barberá-Tomás \& Consoli } \\
\text { (2012) }\end{array}$ & Artificial disc & Patent & Data accumulation \\
\hline
\end{tabular}




\subsubsection{NEW PRODUCT TARGET SETTING}

Product target setting is one of the most essential practices in the early stage of new product development to ensure that the firm pursues the right markets and products from a strategic viewpoint [111]. This involves decisions about the target market, product mix, project prioritization, resource allocation, and technology selection [112]. It should be noted here that, unlike the traditional approach stipulating that the product concept be frozen before detailed product design commences, it may be optimal to pursue multiple product concepts and select the best design through an iterative process [113]-[115].

The body of literature relevant to this topic can be divided into four groups: marketfocused approach, organization-focused approach, operations management-focused approach, and engineering design-focused approach.

The market-focused approach views customer utility as a function of product attributes, hence the emphasis is placed on collecting customers' value propositions for product positioning and pricing by adopting various market research methods [116][119]. Those classic approaches include brainstorming and Delphi [120], morphology (or morphological analysis) [121], and lead users analysis [122]. In addition, recent attempts such as the voice of the customer [123], probe and learn [124], empathic design [125], fuzzy cognitive map [126], and crowdsourcing [127], [128] have been used to derive promising product concepts from consumers' perception as well as underlying behaviors. The key difference between the market-focused approach and engineering design-focused approach is that the former places more emphasis on product concept generation, whereas the latter is more concerned with product concept selection with the determination of specific attribute levels [129]. 
The organization-focused approach is focused at a relatively aggregate level on the determinants of project success from the perspective that a product is an artifact resulting from an organizational process [111]. Consequently, typical subjects include development team organization (functional, project, or matrix) [130], [131], team staffing [132], [133], team performance measurement [134]-[136], arrangement of the development team [137]-[139], infrastructure and training [140]-[142], and development stage-gate [143]-[145]. Recently, the critical roles of leadership and of communication and conflict management training are receiving extensive attention as strategies for overcoming the challenges to team effectiveness in new product development [146], [147].

The operations management-focused approach can be viewed as a stream of literature with regard to the topic of financial and business environment analysis for new product development [148], [149]. This approach has a viewpoint that a product is a sequence of development and/or production process steps with the goal being to achieve "high efficiency" across the steps [111]. The topics, therefore, mainly focus on capacity utilization [150], process performance [151], development sequence and schedule [152], supplier and material selection [153], etc. Specifically, one of the most popular models is capital asset pricing model (CAPM), which attempts to optimize the level of return at the lowest possible level of risk within a product portfolio [154]. Failure mode and effects analysis (FMEA), similarly, provides a framework to identify actions that could eliminate or reduce the likelihood of the potential failure of a product or process [155], [156]. More recently, Markeset and Kumar's study proposed the integrated model of RAMS, i.e., reliability, availability, maintainability and supportability employing the life cycle cost 
(LCC) analysis within the stage gate model for project and work process management [157].

Much of the engineering design-focused approach is at a more detailed level of abstraction with the focus being the individual product or specific market [111]. The perspective of this approach is that a product is a complex assembly of interacting components [134]. Perhaps the most known method is conjoint analysis, which attempts to identify the ideal combination of product size, shape, configuration, function, and dimensions [158]. Furthermore, recent attention to the product categorization has been enhanced by benchmarking studies in an attempt to identify distinct combinations of product attributes. An initial work related to this product-focused approach may be found in Doyle and Green's study which used a widely known benchmarking technique, data envelopment analysis (DEA), to identify homogeneous product groups, i.e., competitors, as well as market niches [21]. Specifically, they applied DEA to classify printers by ordering them from broad to niche based on the number of times each printer appears in others' reference sets. In a similar vein, Seiford and Zhu developed measures for products' attractiveness and progress by separating context-dependent frontiers [22]. Further, Po et al. showed how this product feature-based clustering can be used for decision makers to know the changes required in product design so the product can be classified into a desired cluster [23]. Amirteimoori and Kordrostami later extended this approach to take the size of products into account, thereby comparing products grouped by scale [24]. In addition, Amin et al. clarified the role of alternative optimal solutions in the clustering of multidimensional observations from the DEA approach [159]. Most recently, Dai and 
Kuosmanen proposed a new approach that can take into account the cluster-specific efficiency rankings as well as stochastic noises [25].

Table 11 Summary of literatures on the new product target setting

\begin{tabular}{|c|c|c|c|}
\hline Classification & Perspective on product & \multicolumn{2}{|c|}{ Methods (or main topics of discourse) } \\
\hline $\begin{array}{l}\text { Market- } \\
\text { focused }\end{array}$ & $\begin{array}{l}\text { A product is a bundle } \\
\text { of attributes }\end{array}$ & $\begin{array}{l}\text { - Brainstorming } \\
\text { - Delphi } \\
\text { - Morphology } \\
\text { - Lead user analysis }\end{array}$ & $\begin{array}{l}\text { - Voice of customer } \\
\text { - Probe and learn } \\
\text { - Empathic design } \\
\text { - Fuzzy cognitive map } \\
\text { - Crowdsourcing }\end{array}$ \\
\hline $\begin{array}{l}\text { Organization- } \\
\text { focused }\end{array}$ & $\begin{array}{l}\text { A product is an artifact } \\
\text { resulting from an } \\
\text { organizational process }\end{array}$ & $\begin{array}{l}\text { - Development team } \\
\text { organization } \\
\text { - Team staffing } \\
\text { - Performance } \\
\text { measurement }\end{array}$ & $\begin{array}{l}\text { - Team arrangement } \\
\text { - Infrastructure and } \\
\text { training } \\
\text { - Development state- } \\
\text { gate } \\
\text { - Leadership and } \\
\text { communication }\end{array}$ \\
\hline $\begin{array}{l}\text { Operations } \\
\text { management- } \\
\text { focused }\end{array}$ & $\begin{array}{l}\text { A product is a sequence } \\
\text { of development and/or } \\
\text { production process } \\
\text { steps }\end{array}$ & $\begin{array}{l}\text { - Capacity utilization } \\
\text { - Process performance } \\
\text { - Development } \\
\text { sequence and schedule } \\
\text { - Supplier and material } \\
\text { selection }\end{array}$ & $\begin{array}{l}\text { - } \text { Capital asset pricing } \\
\text { model (CAPM) } \\
\text { - Failure mode and } \\
\text { effects analysis } \\
\text { (FMEA) } \\
\text { - Reliability, } \\
\text { availability, } \\
\text { maintainability and } \\
\text { supportability } \\
\text { (RAMS) } \\
\text { - Life cycle cost } \\
\text { (LCC) }\end{array}$ \\
\hline $\begin{array}{l}\text { Engineering } \\
\text { design- } \\
\text { focused }\end{array}$ & $\begin{array}{l}\text { A product is a complex } \\
\text { assembly of interacting } \\
\text { components }\end{array}$ & $\begin{array}{l}\text { - Conjoint analysis } \\
\text { - Data envelopment } \\
\text { analysis (DEA) }\end{array}$ & \\
\hline
\end{tabular}




\subsection{TeChnology Assessment Methods}

\subsubsection{QUALITATIVE MODELS}

\section{A. DELPHI AND EXPERT OPINION}

The Delphi technique was developed during the 1950s by workers at the RAND Corporation and became publicized by Dalkey and Helmer in 1963 [120]. Delphi's consensus building process is derived by a series of intensive questionnaires interspersed with controlled opinion feedback. It is believed that as a result of contacting the panelists by letter (or electronic correspondence), the disturbing factors of group discussion such as bandwagon effect produced by the majority opinion can be minimized [3]. Delphi may ask experts about direct forecasts of technological parameters or likelihoods of future events; however, it was originally intended for use in judgment and forecasting situations in which pure model-based statistical methods are not practical or possible [160]. Thus, Delphi is applied to the most important task of setting up goals on higher levels such as social, national, and corporate goals. Recent applications include emerging infectious animal diseases [161], health and social care [162], Basque university systems [163], and essential drugs needed for quality care of the dying [164].

\section{B. SCENARIO ANALYSIS}

Scenario analysis postulates a set of imaginative descriptions that can encompass the plausible range of future aspects [6]. This technique particularly attempts to set up a synoptic view of as many developments as can be grasped and as may appear relevant to an experimental simulation of a possible reality. Kahn asserted the importance of scenario mapping by saying that "a specific estimate, conjecture, or context, even if it is 
later shown to have serious defects, is often better than a deliberate blank which tends to stop thought and research [165]."

This technique is usually integrated with other forecasting models not only to identify a firm basis of possibilities but also to investigate the impact of technology interactions under the various conditions. Recent hybrid applications of scenario analysis include Nowack et al.'s Delphi-based model [166], Winebrake and Creswick's analytical hierarchy process (AHP)-based model [167], Kok et al.'s participative back casting-based model [168], and Jetter and Schweinfort's fuzzy cognitive map (FCM)-based model [169].

\section{RISK ASSESSMENT AND ENVIRONMENTAL IMPACT ANALYSIS}

Risk analysis pays particular attention to the negative impact of technologies on social institutions and critical infrastructure [170]. Linkov et al. surveyed the comparative risk assessment (CRA), multi-criteria decision analysis (MCDA), and adaptive management methods applicable to environmental remediation and restoration projects and asserted that it is required to shift from optimization-based management to an adaptive management paradigm for the conservation of the ecosystem [171]. Recently, as an attempt to distinguish and categorize the potential risk in advance, more attention has been paid to developing the predictive models. As an example, Kolar and Lodge developed an ecological risk assessment model to evaluate the risk of alien species for nonagricultural systems [172].

In a similar vein, environmental impact analysis (EIA) has become an important and often obligatory part of today's technology assessment [173]. Ramanathan's study applied a multi-criteria model to capture the perceptions of stakeholders on the relative 
severity of different socio-economic impacts, which will help the authorities in prioritizing their environmental management plan [174]. Van der Werf and Hayo compared 12 indicator-based approaches to assess the environmental impact at the farm level and provided a set of guidelines for the proper application of EIA [175].

\section{RELEVANCE TREES}

The notion of relevance trees was first proposed by Churman et al. in an attempt to aid decision making in general industrial contexts [3], [176]. The qualitative relevance trees are frequently employed in conjunction with scenario analysis to estimate the significance of criteria. The process can be simplified as follows. First, the relevance trees, namely hierarchical decision models, are constructed to assess missions, objectives, goals, strategies, activities, etc. From the scenario analysis, a number of criteria are derived for each level of the relevance trees. Then, the weights and significance numbers are estimated on the basis of the identified scenarios. Finally, the decision matrices are computed to provide a set of alternative actions for each scenario.

The extended applications using relevance tree can be found in recent studies, including Ghiculescu et al.'s model integrating the decision structure with customer matrix (CM) [177] and Manuel and Pretorius's model using criteria derived from relevance trees as inputs of neural network [178]. 


\section{E. MORPHOLOGY}

The morphology (or morphological method) was developed by Zwicky in 1962 [121] in an attempt to deduce all of the solutions of a given definite problem. The method proceeds as follows:

1. The statement of the problem, i.e., the object of the morphological device, is made.

2. The precise definitions of the class of devices are elaborated.

3. Related parameters with sub-elements are grouped as matrices and listed for connection.

4. The alternative solutions are obtained as chains of selected elements from each matrix.

5. Determine the performance values of all of the derived solutions.

6. Select the particularly desirable solutions and their realization.

This process provides a framework for thinking in basic principles and parameters, which is growing in importance, even if practiced in a disordered or ad hoc fashion [3].

Recent developments of morphology technique tend to be in conjunction with data mining approaches with the current heavy interests on network analysis. Examples can be found in Feng and Fuhai's study [179] and Jun et al.'s study [180] that used patent-based morphological mapping, and Yoon et al.'s study [181] that developed the text mining morphology analysis. 


\section{F. ANALOGY}

As a research technique, analogies have been mainly applied in the social sciences [3]. Nonetheless, it may improve the anticipatory insight, especially when quantitative methods suffer from the absence of sufficient data but there exist analogous events in history. The classic application can be found in Bruce's study of "The Railroad and the Space Program-An Exploration in Historical Analogy” in 1965 [182]. The study sought to test the feasibility of using railroad development in a systematic way to forecast space program development. The historical analogy method, however, tends to neglect the political, social, and philosophical impact, thereby often providing unsatisfactory forecasts. A recent application of Goodwin et al. [183], which conducted a comparative analysis of four different forecasting methods using analogous time series data for a sales forecast of a new product, also concluded that using an analogy led to higher errors than the parameters estimated from small but actual data.

\section{G. CAUSAL MODEL}

A causal model considers the explicit cause-and-effect relationships that affect the growth of technology systems [6]. Therefore, this technique relies on the assumption that the relevant variables and their linkages are known and can be described in a structural model. However, due to the lack of information, the use of causal modeling is limited to forecasting adoption or diffusion of innovations where the related parameters can be measured [50], [184]-[188]. 


\subsubsection{QUANTITATIVE MODELS}

\section{A. DECISION ANALYSIS}

Multi-criteria decision making (MCDM) methods have been widely applied to the technology assessment to integrate qualitative values into quantitative factors of technologies. The traditional applications of decision analysis may start from the check lists [189], expected value model [190], and stochastic success evaluation model [191]. Recent developments of decision analysis model on technology assessment are facilitated by more advanced multi-criteria decision theories. Ondrus and Pigneur investigated the potential of near field communication (NFC) as an upcoming technology for mobile payments [192]. Their study conducted comparative analysis on an expert panel and showed that the Swiss industry was enthusiastic about adoption of this technology. Daim et al. used an applied hierarchical decision model to identify the optimal design characteristics for the U.S. Northwest off-shore wind turbine [193]. As an application in the service industry, Tang and Tzeng applied a hierarchy fuzzy MCDM to examine critical environmental factors relevant to Internet commerce in changes in the international marketing environment from the perspective of business activity, socioeconomics, and information management [194]. 


\section{B. ECONOMIC ANALYSIS (COST-BENEFIT ANALYSIS)}

Economic analysis (or cost-benefit analysis), which is not confined to the technology assessment method, is frequently used to translate estimates resulting from technological forecasting into economic terms with the purpose of evaluating the impact of a technological innovation. This implies that it should be possible to quantify the forecasts in monetary terms, although this may be a challenge where qualitative aspects such as social goals or environmental impacts are mainly concerned.

The application of economic analysis to technology assessment stems from the crude forms of a return on investment or a discounted cash flow to a gain factor considering technical feasibility or a project acceptability index considering past experience [195]. Moslehi and Kumar delineated the optimistic vision of the smart grid not only from its technical promises but also from the expected benefits that significantly outweigh the estimated costs [196]. Friedewald and Oliver analyzed the economic aspect of the ubiquitous computing and claimed that the cost barrier of RFID transponders and system integration should be overcome [197].

\section{SYSTEM DYNAMICS}

System dynamics is defined as a simplified representation of the structure and dynamics of part of the real world [6]. As an attempt to apply dynamic modeling to technology assessment, Forrester proposed the concept of system dynamics for complex systems [198]. Therefore, system dynamics has been particularly popular for shaping the dynamic ecosystem of the future technologies and their diffusion patterns by taking feedback, i.e., non-linear behaviors among entities, into account. 
Daim et al. applied system dynamics to the fuel cell industry and found that the adoption rate would be increased as a consequence of government policies and supply/demand relations [199]. Maier developed a new product diffusion model using system dynamics to incorporate competition and to map the process of substitution among successive product generations [200]. Suryani et al. constructed a system dynamics model to forecast air passenger demand and to evaluate some policy scenarios related with runway and passenger terminal capacity expansion to meet the future demand [201].

\section{EXTRAPOLATION}

Extrapolation models employ mathematical and statistical techniques to extend time series data into the future under the assumption that the past conditions and trends will continue in the future more or less unchanged [6]. Since estimation is a data-based forecast, it requires a sufficient amount of good data to be effective. The next section provides a focused review on the frequently used extrapolation models that can deal with multi-attribute assessment.

Figure 4 classifies various technology assessment methods in two dimensional plots. While the qualitative approaches tend to focus more on eliciting multiple perspectives from the knowledge sources (e.g., expert panels, history, etc.), the quantitative approaches place more emphasis on drawing meaningful findings by analyzing the numerical data. It is not surprising that technology focused approaches tend to be quantitative, whereas society focused approaches are mostly qualitative. 


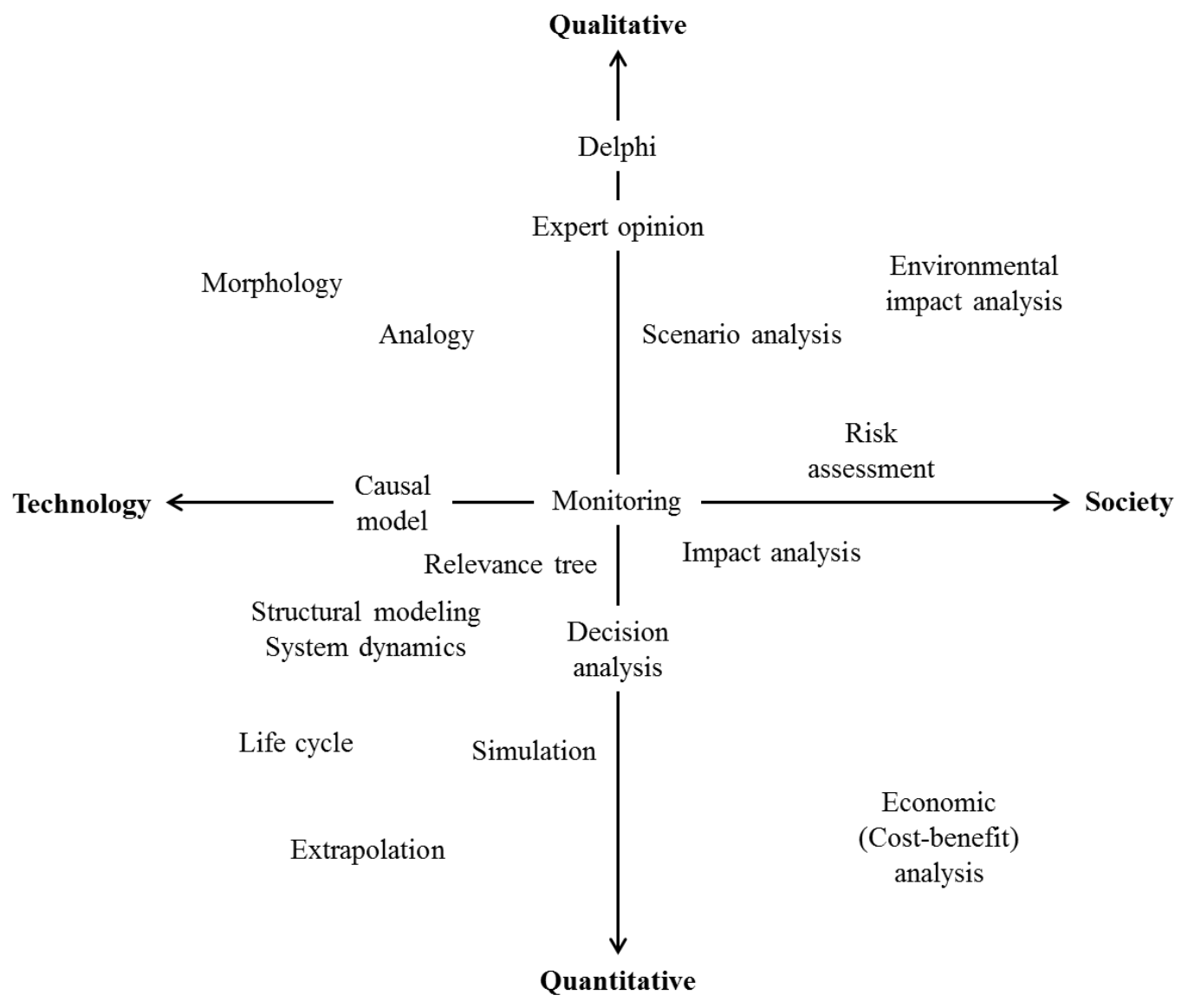

Figure 4 Technology assessment methods 


\subsection{Focused Review on Multi-attribute Extrapolation Methods}

As technology becomes even more sophisticated, there are few technologies that truly possess only a single technical capability. The rate of change also varies over time, being affected by the maturity levels of component technologies. This structural complexity makes today's forecasting even more challenging, which leads to the question: how to combine growth patterns of each attribute to describe the multi-objective technology systems? There are three different approaches to tackle this problem: intuitive modeling, parametric frontier modeling, and non-parametric frontier modeling.

\subsubsection{INTUITIVE MODELS}

The intuitive models are used to combine multiple technology attributes into a single technology measure, often where no physical basis exists to do so [38]. Therefore, these models are often technology specific, non-unique and highly subjective.

\section{A. SCORING MODEL}

Martino suggested distinguishing overriding variables, tradable variables, and optional variables to construct an appropriate scoring model [202]. His example of the fighter jet scoring model $^{2}$ is shown below:

Score $=\frac{\text { Maneuver } * \text { Availability } * \text { Range } * \text { Payload } * \text { Speed } * \text { Avionics }^{2} * \text { Weapons }^{2}}{1+\text { Takeoff roll }}$

Once the scoring model is obtained, it becomes possible to estimate the overall score of the future technologies by extending the historical trend. However, while the scoring

\footnotetext{
${ }^{2}$ The weights and the tradeoff coefficients were determined by an Air Force officer's subjective judgment.
} 
model provides a composite measure so that each technology system can be put on a common basis, it is not capable of capturing the necessary information to simultaneously evaluate each system attribute relative to the remaining attributes.

\section{B. TECHNOLOGY DEVELOPMENT ENVELOPE}

Technology development envelope (TDE) was originally developed by Gerdsri to identify an optimum technology development path as a roadmapping method [203]. The procedure consists of six steps:

Step 1: Technology forecasting to identify emerging alternatives.

Step 2: Technology characterization to establish evaluation criteria.

Step 3: Technology assessment on identified alternatives based on criteria.

Step 4: Hierarchical modeling to determine the relative importance of criteria.

Step 5: Technology assessment to determine the relative value of alternatives.

Step 6: Formation of TDE to establish an optimum development path.

Within this process, TDE constructs a hierarchical decision model (HDM) to determine the relative importance of emerging technologies aligned with the organization's objectives. Technologies having the highest value in each time period represent the most preferred technology alternatives. In this sense, the path connecting those technologies from one period to another becomes an optimal technology development roadmap.

However, since the technology assessment process in TDE is predicated upon HDM, multiple perspectives on technology attributes are to be averaged within the process of obtaining a single ranking of technology alternatives for each period. That is, 
combinatorial values derived from different levels of technology attributes are supposed to be represented by a single weighting scheme aggregated from a panel of experts' opinions. This becomes a critical issue to identify the "better" technology when the market segments exist as having particular customers with differing value propositions on technology systems.

\subsubsection{PARAMETRIC FRONTIER MODELS}

The parametric frontier approach is characterized by being defined a priori with several assumptions on random noise and efficiency distribution in an attempt to approximate the ideal relationship between inputs and outputs. Since actual observations are to be compared with generalized production possibilities, the measurement is defined to be "functionally relative."

\section{A. Planar Frontier Model (Hyper-Plane)}

The planar frontier model (or hyper-plane method) was first introduced by Alexander and Nelson [204]. They assumed that the movement of the tradeoff surface is describable by a smooth and monotonic function. The function to be estimated by a multiple regression is given as:

$$
t=F\left(P_{1}, \ldots, P_{n}\right)
$$

where $t$ is the introduction date of a system, and $P_{i}$ denotes the technical capabilities.

Specification of the functional form and determination of the coefficients of the equation provide a measure of average technological trend over time. For example, Lim 
et al. applied a planar frontier model to develop the wireless protocol forecasting model. The resulting equation is shown as follows [205]:

$$
\begin{array}{r}
\text { Forecasted date of introduction }=1984.411-2.532 *(\text { Linearized }) \text { Bandwidth } \\
+6.651 *(\text { Linearized }) \text { Bitrate }
\end{array}
$$

A major advantage of this approach is a simple implementation based on multiple regression analysis, which allows straightforward interpretation of the results. Also, regression diagnostics can be used to select significant parameters in explaining the technological progress. However, the linearity assumption underpinning this approach can be a disadvantage at the same time. In particular, the planar frontier assumes a constant rate of change without considering acceleration from increased engineering efforts or deceleration as the system approaches the physical limit. Therefore, this approach may result in an inaccurate forecast, particularly when the technological systems experience architectural transition. Furthermore, this approach may not be applicable to large systems, as there could be difficulties with the use of technical parameters arising from the tradeoffs between different component systems [206]. Presupposition of treating an ordinal index as a cardinal measure, i.e., using release date as a proxy for the level of technology, should also be justified to employ this approach [207]. 


\section{B. CoRRECTED ORDINARY LEAST SQUARES}

Aigner and Chu introduced a corrected ordinary least squares (COLS) method to construct a deterministic and parametric production function by extending the ordinary least squares method [208]. This process first estimates unknown parameters $\beta$ using the ordinary least squares method:

$$
\min _{\beta} \sum_{k}\left(y^{k}-f\left(x^{k} ; \beta\right)\right)^{2}
$$

where $\left(x^{k}, y^{k}\right)$ denotes actual $k$ observations and $f$ is a production function. Then, the model finds the smallest possible correction by introducing an additional coefficient $\beta_{00}$ to ensure that all observations are placed below the production frontier with the maximum error term [209]:

$$
\beta_{00}=\max \left\{y^{k}-f\left(x^{k} ; \hat{\beta}\right) \mid k=1, \ldots, K\right\}
$$

Once the production function is estimated, the efficiency measurement for each observed production can be made by comparing them to the maximum (minimum) possible output (input) for a given input (output) along with a desired directional distance function.

Since COLS has its root in statistical principles, i.e., the maximum likelihood, the frontier is constituted to represent the general pattern of actual observations without taking noise into account [26]. That is, any variation in the dataset, including possible noise, is considered to contain significant information about the efficiency. Therefore, this method may not be appropriate when there is a need to identify the underlying pattern of production possibilities without the impact of the random noise. 


\section{STOCHASTIC FRONTIER ANALYSIS}

As another variation of the ordinary least squares method, stochastic frontier analysis (SFA) was introduced by Aigner et al. in an attempt to construct the stochastic and parametric production function [210]. That is, the SFA model includes both a stochastic error term and a parametric inefficiency term defined by a frontier curve. The basic model, simplified by Bogetoft [26], can be shown as follows:

$$
y^{k}=f\left(x^{k} ; \beta\right)+v^{k}-u^{k}, \quad v^{k} \sim N\left(0, \sigma_{v}^{2}\right), \quad u^{k} \sim N_{+}\left(0, \sigma_{u}^{2}\right), \quad k=1, \ldots, K
$$

where $v^{k}$ denotes the noise, $u^{k}$ the inefficiency, and $N_{+}$a half-normal distribution.

Compared to COLS, SFA distinguishes noise with inefficiency (see Fig. 5). Therefore, efficiency scores from SFA tend to be higher than COLS or other deterministic frontier models. This characteristic would be appropriate when the dataset suffers from random variations, and therefore outliers need to be detected in the process of frontier formation. On the other hand, this might restrict the perspective of identifying various tradeoffs that can represent the distinct production possibilities.

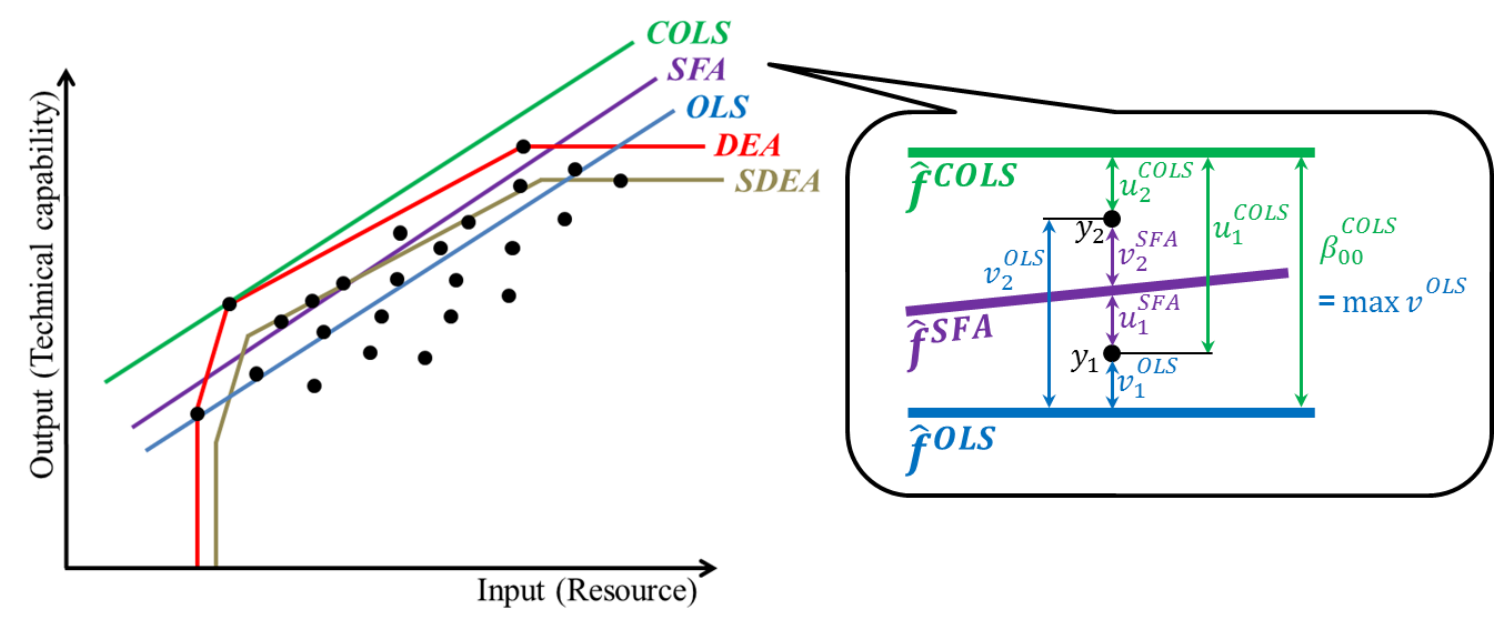

Figure 5 Frontier models in input-output space (modified from [26]) 


\section{ELLIPSOID FRONTIER MODEL}

As a non-linear frontier model, Dodson proposed an ellipsoid frontier model to quantify the technological advance in relation to the state of the art (SOA) surface [211], [212]. This model attempts to fit the technology frontier into an ellipsoid functional form from which tradeoffs among attributes can be explained (see Fig.6). Dodson's measure of technological advance is defined as $\left(\gamma^{2}-1\right)$, where

$$
\gamma=\frac{\text { Distance from origin to data point being assessed }}{\text { Distance from origin to projection on SOA frontier }}
$$

Martino later extended Dodson's model to allow use of it in any order [213]. Martino's generalized ellipsoid model is given as follows:

$$
\sum_{i}\left(\frac{X_{i}}{a_{i}}\right)^{n}=1
$$

where $n$ is the order of the ellipsoid, $X_{i}$ the value of the $i$ th technical capability, and $a_{i}$ the intercept of the ellipsoid on the $i$ th axis.

Martino also suggested using the mean absolute deviation rather than the mean squared deviation for the fitting procedure to reduce the effect of extreme values. This allows the fitted frontier surface to be located closer to the median of the observations instead of the mean.

Although this approach can provide a measure to investigate the SOA formation process, the fundamental question remains to be resolved: why the technology tradeoff surface should be following the ellipsoid form? In detail, the ellipsoid frontier model presupposes that the tradeoff of one technical capability being relinquished for the 
advancement of the others can be explained by adopting a predefined functional form rather than by adapting to the data. Dodson's choice of an ellipsoid shape is analytically sound for the representation of a strictly convex surface but may not always be representative.

In addition, the ellipsoid function is limited to explaining the tradeoff between outputs. This requires an assumption that the tradeoff surface is only applicable to the technical capabilities consuming the same amount of inputs such as engineering resources.

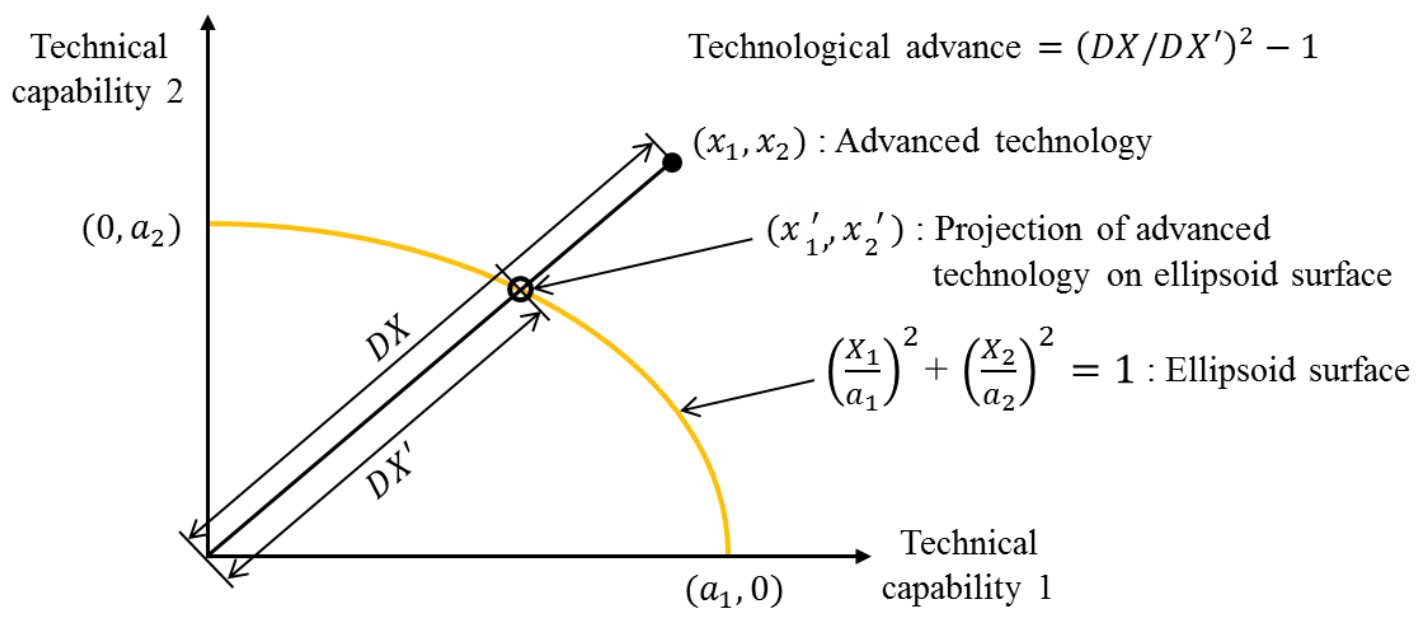

Figure 6 Two-dimensional illustration of ellipsoid frontier (modified from [212]) 


\section{E. MUlti-Dimensional GRoWth Model}

To overcome the limitation of traditional S-curves, being able to deal with only a single attribute for any subject, Danner developed the multi-dimensional growth model (MDGM) to generate an iso-time frontier by combining individual technology growth curves [32]. This approach first decomposes technology systems into individual attribute levels to obtain a proper curve for each subsystem (see Fig. 7.) The iso-time frontier, at which the same levels of technology systems are to be located, is then formulated by combining identified individual growth curves. The resulting model makes it possible to either forecast technical capabilities at a certain point in time or estimate the time by which desired levels of technical capabilities will be operational.

Similar to the planar frontier model, a major limitation to the utility of MDGM is the requirement that all dimensions of technical capability integrated must be statistically independent. This presupposes that the individual time spans required to advance each attribute towards corresponding upper limits can be linearly combined to explain the technology systems' growth rate. However, the higher the complexity of technology systems under evaluation is, the more individual growth rates are likely to be interrelated, hence the generated iso-time frontier without consideration of concurrent advancement would not provide an accurate picture of the feasible combinations of technical capabilities.

Interdependence between technology attributes might also require the modification of established upper limits. It has been frequently observed in highly complex systems that individual upper limits become more challenging to achieve as they tend to restrict 
one another [214], [215]. Therefore, the progress of the iso-time frontier should be guided by adjusted upper limits with the consideration of the architectural complexity involved.

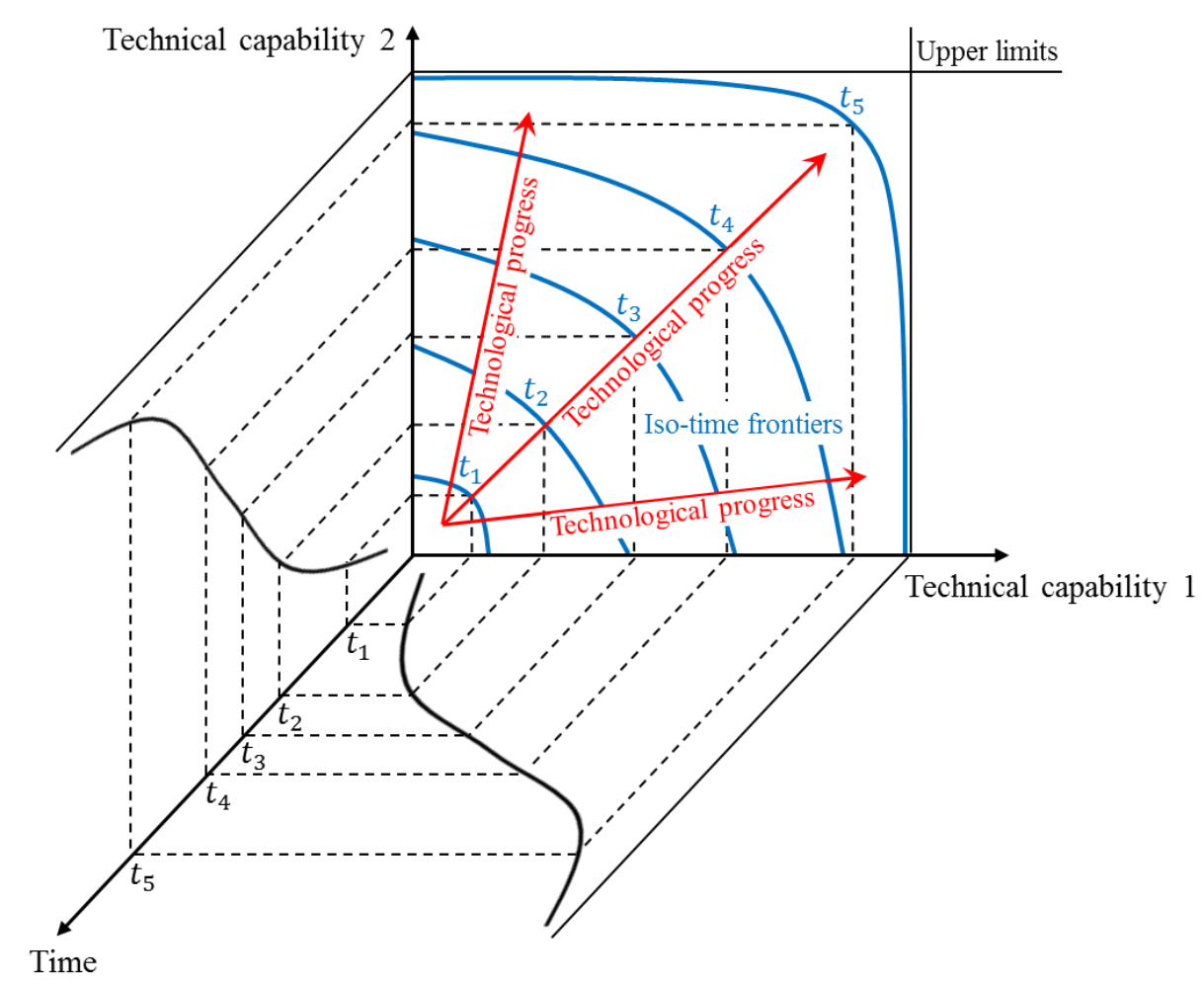

Figure 7 Parametric frontier by MDGM (modified from [32]) 


\subsubsection{NON-PARAMETRIC FRONTIER MODELS}

The non-parametric frontier approach forms the "best practice" frontier without relying on arbitrary functional assumptions. Instead, it maximizes the flexibility to capture various production possibilities observed from the actual data. Since this approach doesn't construct an averaged target with which data points are to be compared, the measurement is defined to be "empirically relative."

\section{A. DATA ENVELOPMENT ANALYSIS}

The original data envelopment analysis (DEA) was proposed by Charnes et al. [216]. As the name of decision making units (DMUs) implies, the efficiency measure in DEA is defined as the ratio of the weighted sum of outputs to the weighted sum of inputs using a freely chosen weighting scheme for each DMU, and as such, the efficiency measure will show them in the best possible light. The ratio form of the dual (multiplier) input-oriented variable returns to scale DEA model can be presented as below:

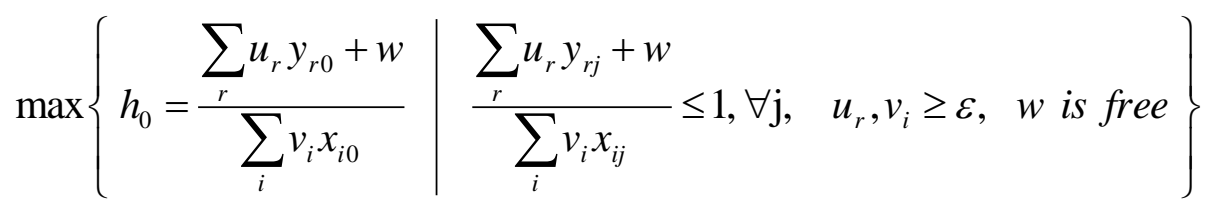

where $h_{0}$ denotes the input-oriented efficiency of DMU being assessed, $u_{r}$ the weight assigned to output $r, v_{i}$ the weight assigned to input $i, x_{i j}$ the $i$ th input variable of DMU $j, y_{r j}$ the $r$ th output variable of DMU $j$, and $w$ the returns to scale (RTS) parameter.

The above input-oriented multiplier model can be readily translated to the primal (envelopment) model, which is shown below as a single-stage theoretical formulation: 


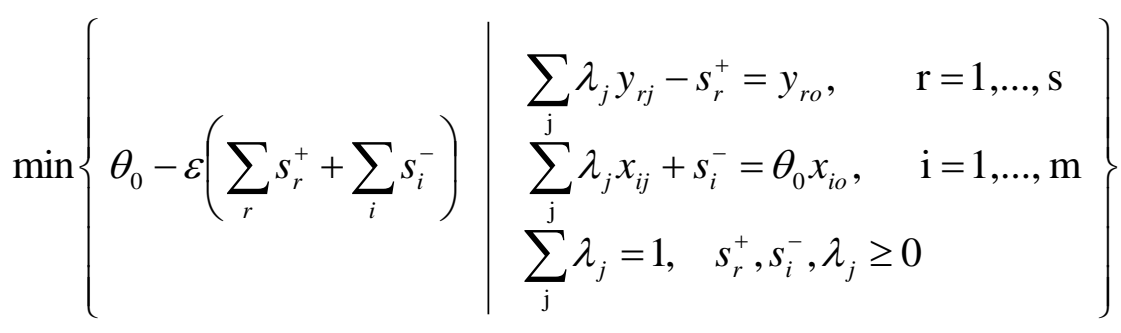

where $\theta_{0}$ denotes the technical input-oriented efficiency, $\lambda_{j}$ the loading factor attached to $\operatorname{DMU} j, s_{r}^{+}$and $s_{i}^{-}$the slacks equal the reduced cost of $u_{r}$ and $v_{i}$ respectively. Note that if the optimal value of $\theta_{0}$ is less than 1 , then $D M U_{0}$ is inefficient in that the model (9) will have identified another production possibility that secures at least the output vector $y_{o}$ but using no more than the reduced input vector $\theta_{o}^{*} x_{o}$. Thus, $\theta_{o}^{*}$ is a measure of the radial input efficiency of $D M U_{0}$ in that it reflects the proportion to which all of its observed inputs can be reduced pro rata, without detriment to its output levels [209].

DEA studies have often examined the changing performance of units over time [217]-[221]. A shorthand notation for a DEA model can be defined as $\theta_{0}^{t}\left(x_{0}^{t}, y_{0}^{t}\right)$ as the efficiency of the DMU o in time period $t$ with input and output characteristics $\left(x_{0}^{t}, y_{0}^{t}\right)$, being measured against the frontier of peers also in time period $t$. A peer compared against units from the following period would then be $\theta_{0}^{t+1}\left(x_{0}^{t}, y_{0}^{t}\right)$. If the value of $\theta_{0}^{t+1}\left(x_{0}^{t}, y_{0}^{t}\right)$ is less than 1.0, then the unit in period $t$ is inefficient relative to units from period $t+1$. If the value of $\theta_{0}^{t+1}\left(x_{0}^{t}, y_{0}^{t}\right)$ is greater than 1.0, then the unit in period $t$ outperforms units from period $t+1$ in some manner and is efficient.

It would be expected that a particular unit's efficiency scores such as $\theta_{0}^{t}\left(x_{0}^{t}, y_{0}^{t}\right)$ and $\theta_{0}^{t+1}\left(x_{0}^{t+1}, y_{0}^{t+1}\right)$ would vary over time, but separating an effect from improved operation of a unit from different conditions affecting all units cannot be determined simply from the 
efficiency scores. Färe et al. introduced a DEA-based Malmquist productivity index (MPI) to measure the technical efficiency change (TEC) and the frontier shift (FS) over time as an extension of the original concept introduced by Malmquist [217], [222]. The inputoriented MPI can be defined as

$$
M P I_{o}=T E C_{0} \cdot F S_{0}=\frac{\theta_{0}^{t}\left(x_{0}^{t}, y_{0}^{t}\right)}{\theta_{0}^{t+1}\left(x_{0}^{t+1}, y_{0}^{t+1}\right)} \cdot\left[\frac{\theta_{0}^{t+1}\left(x_{0}^{t+1}, y_{0}^{t+1}\right)}{\theta_{0}^{t}\left(x_{0}^{t+1}, y_{0}^{t+1}\right)} \frac{\theta_{0}^{t+1}\left(x_{0}^{t}, y_{0}^{t}\right)}{\theta_{0}^{t}\left(x_{0}^{t}, y_{0}^{t}\right)}\right]^{\frac{1}{2}}
$$

where $\theta_{0}^{t}$ denotes DEA efficiency score and $x_{0}^{t}, y_{0}^{t}$ input and output levels at given point in time $t$. Therefore, TEC indicates technical efficiency change between period $t$ and $t+1$ : improves $(<1)$, remains $(=1)$, and declines $(>1$.) In a similar sense, FS measures the amount of frontier shift: regress $(>1)$, no shift $(=1)$, and progress $(<1)$.

To extend the time-series application of DEA into technological forecasting, Inman developed a measure to quantify the rate of frontier expansion by which the arrival of the following DMUs can be estimated [223]. Specifically, his method, technology forecasting using data envelopment analysis (TFDEA), establishes the envelopment, i.e., SOA technology frontier, using the data points identified as relatively efficient from DEA (see Fig. 8). Note that the frontier is a set of convex combinations formed by SOA technologies, hence it's not a curved surface but a piecewise linear combination. The tradeoffs between technical capabilities can be considered as a radial improvement within this frontier space. The TFDEA iterates the frontier formation process over time to track the rate of frontier shift. This momentum of progress is then used to make a forecast for the future technologies (DMUs.)

Unlike the iso-time frontier from MDGM, the frontier constructed by TFDEA typically consists of multiple vintages of SOA technologies. This allows the model to 
specify the individual timing, i.e., effective time, of any points on the frontier according to the corresponding tradeoff surface. This enables TFDEA to identify the starting point of each forecasting target from which their best forecast can be made. Lim et al. examined how this approach could improve the forecasting accuracy compared to a planar frontier model in which the constant baseline, i.e., the regression constant, is assumed for all forecasts [205].

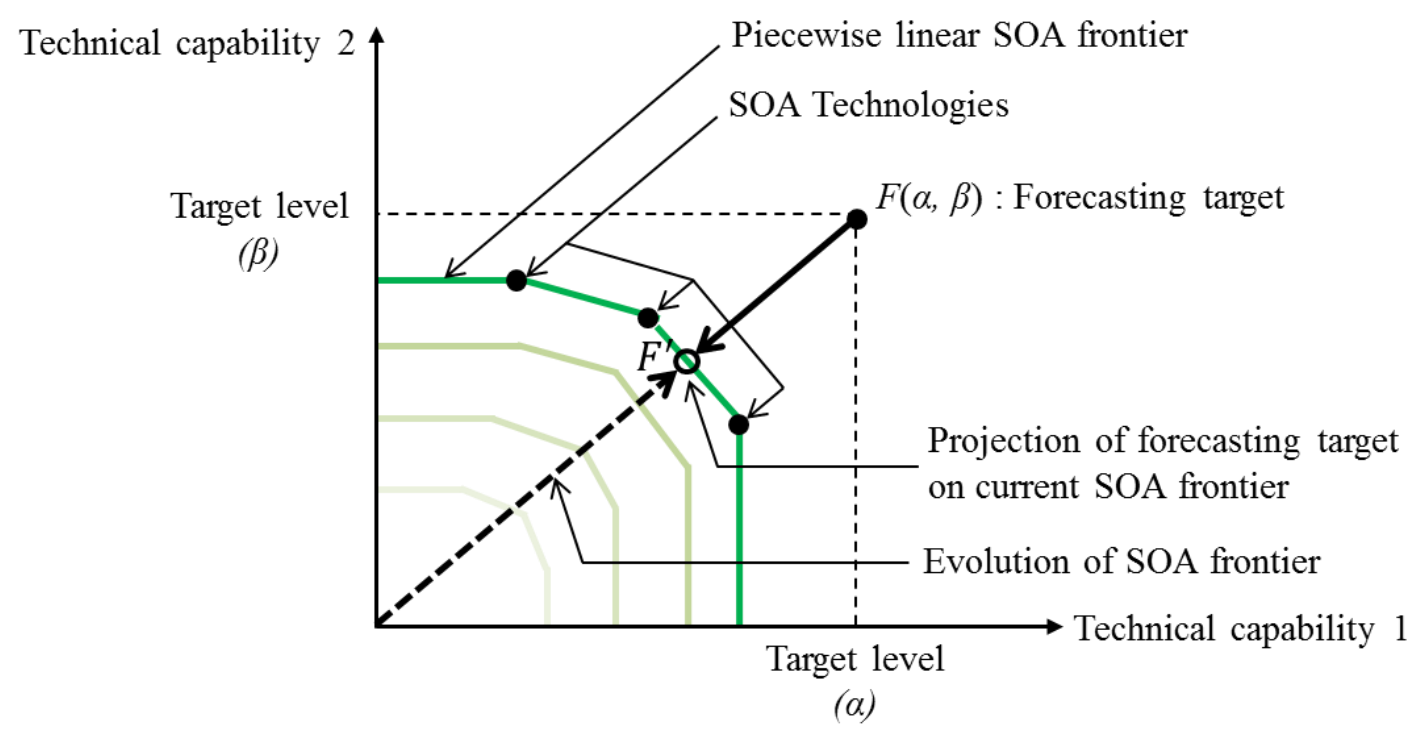

Figure 8 Non-parametric frontier by TFDEA

In spite of additional benchmarking information provided by DEA within TFDEA process, traditional TFDEA doesn't utilize this information when it comes to a consolidation of rate of changes (RoCs) captured from the past technologies. In fact, it simply employs an average value to make a forecast for future technologies regardless of their unique characteristics. That being said, RoCs captured from the surpassed technologies are simply aggregated to represent the technological progress as a whole. 
This might overlook the unique growth patterns captured from different tradeoff surfaces. Consequently, it was shown at times in previous applications that forecasting based on a single aggregated RoC did not consider the unique growth patterns of each technology segment, which resulted in a conservative or aggressive forecast [224], [225]. This issue, in particular, becomes more visible when the application area contains distinct progress patterns identified from multiple technology segments. Therefore, it is necessary to incorporate the notion of segmented RoC into the forecasting procedure so that each forecasting target can be subject to the individualized RoC that best reflects the potential growth rate of analogous technologies.

It has been occasionally observed from the past applications that TFDEA may suffer from instances of infeasible super-efficiencies when variable returns to scale (VRS) was assumed. In theory, this is also a problem for the input-oriented decreasing returns to scale (DRS) model and output-oriented increasing returns to scale (IRS) model [27]. This problem results in failure to make a forecast for the target technology since the model is unable to measure the superiority of corresponding technology compared to current SOA technologies. Note that the constant returns to scale (CRS) model is also susceptible to this problem when zero data is included in any input variables [27]. However, this is rare in actual applications since it indicates heterogeneous DMUs or technologies [209].

The problem of infeasibilities in the super-efficiency model can be attributed to the inherent characteristics of a non-parametric frontier since this approach identifies the production possibilities without spanning unobserved regions. Especially under VRS, DEA constitutes the frontier purely based on observed DMUs and, therefore, tradeoffs in uncharted regions remain unknown. This renders forecasting targets subject to those 
unknown regions impossible to be projected from the current SOA frontier in a radial manner.

\section{B. StochastiC (CHANCE-CONSTRAINED) DATA ENVELOPMENT ANALYSIS}

Land et al. proposed a data envelopment analysis model that can deal with stochastic variability in inputs and outputs, which evolved from the earlier technique called chanceconstrained programming developed by Land et al. [226], [227]. The standard inputoriented model is presented below:

$$
\begin{array}{ll}
\min \theta-\varepsilon\left(\sum_{r} s_{r}^{+}+\sum_{i} s_{i}^{-}\right) \\
\text {s.t. } & E\left(\sum_{j} \lambda_{j} \cdot y_{r j}-y_{r 0}\right)-F^{-1}(1-K) \sigma_{0}-s_{r}^{+}=0, \quad r=1, \ldots, s \\
& \sum_{j} \lambda_{j} \cdot x_{i j}+s_{i}^{-}=\theta \cdot x_{i 0}, \quad i=1, \ldots, m \\
& s_{r}^{+}, s_{i}^{-}, \lambda_{j} \geq 0, \quad \forall r, i, j \\
& \theta \text { unrestricted in sign }
\end{array}
$$

where $\theta$ denotes radial input contraction factor, $s_{r}^{+}$and $s_{i}^{-}$slack variables, $E$ the mathematical expectation, $F$ the distribution function of the standard normal distribution, $\sigma$ the standard deviation of best practice output minus observed output, i.e., s.d. $\left(\sum_{j} \lambda_{j}\right.$. $\left.y_{r j}-y_{r 0}\right), \lambda_{j}$ loading factor, $x_{i j}$ the $i$ th input variable of DMU $j, y_{r j}$ the $r$ th output variable of DMU $j, K$ threshold fraction allowing hyper-efficiency [228].

Formulation (10) minimizes the contraction factor $\theta$, subject to two sets of constraints. 
First, the observed outputs must not exceed best practice outputs more often than probability of $K$. For example, in the case of $K=0.01$, only $1 \%$ or less of DMUs will do better than the DMU being assessed. That is, $K$ indicates the fraction of DMUs being located above the frontier in Fig. 5. This constraint can be simplified as below [26]:

$$
P\left(\sum_{j} \lambda_{j} \cdot y_{r j} \leq y_{r 0}\right) \leq K, \quad r=1, \ldots, s
$$

where $P$ denotes the mathematical probability.

Second, there is a deterministic constraint for inputs stating that the benchmark being compared must not use more inputs than the reduced inputs, $\theta x_{i 0}$.

The efficiency score is determined by the above model for each DMU, where $\theta^{*}$ denotes optimum chance-constrained DEA efficiency score, such that sub-efficient $\left(\theta^{*}<\right.$ 1 or $\theta^{*}=1$ with non - zero slacks $)$, efficient $\left(\theta^{*}=1\right.$ with zero slacks $)$, and hyperefficient $\left(\theta^{*}>1\right)$ [226].

Similar to the difference between COLS and SFA, this approach is able to separate noise from inefficiency included in the traditional DEA. However, this procedure should be supported by statistical requirements such as input and output variables that are known to be normally distributed. Information about joint probability distributions of the random variables should also be justified to make the resulting efficiency reliable [229]. 


\subsection{Summary of Critical Review}

Table 12 summarizes technology assessment models that can take tradeoffs from multiple technology attributes into account. Aside from intuitive models, the primary difference between parametric frontier models and non-parametric frontier models is how much weight is put on uncertainty versus flexibility. The former approach places a higher importance on the uncertainty by being defined a priori except for a finite set of unknown parameters that are estimated from the data [26]. Therefore, a parametric approach tends to be robust to extreme points by filtering them with a predefined "general" pattern. The latter approach, in contrast, purely adapts the SOA frontier to data without being shaped a priori, which renders the resulting frontier to be a piecewise linear combination rather than a curved surface. This property makes it possible for the non-parametric approach to take full advantage of the distribution of the dataset without relying on statistical corrections.

Among the investigated multi-attribute evaluation models, the non-parametric frontier approach shows the favorable features that make it possible to take account of technological segmentation by classifying generated frontier facets. In particular, the deterministic model may be preferred to the stochastic model in the practical sense that the flexible nature enables a versatile application by not being restricted to statistical conditions. It is worth noting here that, by definition, the shape of the SOA frontier indicates system tradeoffs among technical capabilities. That being said, while the parametric approach presupposes that the shape of SOA surface, namely system tradeoffs, would not change over time, the nonparametric approach attempts to elaborate its changes in each time period with the given data. One can argue that a lack of 
stochastic estimations results in a mean structure of technological innovation degraded by random noises, i.e., extreme technologies. However, deterministic frontier analysis, which takes advantage of superior technologies rather than being averaged by mediocre or inferior technologies, has shown its usefulness in a wide range of technological forecasting studies [225], [230]-[232]. In addition, it is expected that robustness of the model can be partly compensated by the frontier segmentation approach by treating distinguished groups of technologies separately. Meanwhile, the infeasibility problem must be dealt with to guarantee the coherent and complete forecasting process.

The subsequent sections will elaborate how the new approach is formulated with respect to identified research questions.

Table 12 Summary of multi-attribute technology assessment models

\begin{tabular}{|c|c|c|c|}
\hline & \multirow{2}{*}{ Intuitive method } & \multicolumn{2}{|c|}{ Frontier analysis method } \\
\hline & & Parametric frontier model & Non-parametric frontier model \\
\hline Pros & $\begin{array}{l}\text { - High flexibility in } \\
\text { model building }\end{array}$ & $\begin{array}{l}\text { - Capable of detecting } \\
\text { outliers }\end{array}$ & $\begin{array}{l}\text { - Free from parametric } \\
\text { requirements } \\
\text { - Capable of identifying } \\
\text { frontier segments }\end{array}$ \\
\hline Cons & $\begin{array}{l}\text { - } \text { Relies on } \\
\text { subjective opinions } \\
\text { - No consideration } \\
\text { of multiple } \\
\text { tradeoffs }\end{array}$ & $\begin{array}{l}\text { - Difficult to estimate } \\
\text { required parameters } \\
\text { - Sensitive to } \\
\text { multicollinearity }\end{array}$ & $\begin{array}{l}\text { - Sensitive to noises/extremes } \\
\text { - Susceptible to infeasibility }\end{array}$ \\
\hline Model & $\begin{array}{l}\text { - Scoring model } \\
\text { - TDE (Technology } \\
\text { Development } \\
\text { Envelope) }\end{array}$ & $\begin{array}{l}\text { - Planar model } \\
\text { (Hyper-plane) } \\
\text { o COLS (Corrected } \\
\text { Ordinary Least Squares) } \\
\text { o SFA (Stochastic Frontier } \\
\text { Analysis) } \\
\text { - Ellipsoid frontier } \\
\text { - MDGM (Multi- } \\
\text { Dimensional Growth } \\
\text { Model) }\end{array}$ & $\begin{array}{l}\text { - TFDEA (Technology } \\
\text { Forecasting using Data } \\
\text { Envelopment Analysis) } \\
\text { O SDEA (Stochastic Data } \\
\text { Envelopment Analysis) }\end{array}$ \\
\hline
\end{tabular}




\section{MODEL DEVELOPMENT}

\subsection{Conceptual Framework}

\subsubsection{Segmented Rate of Change}

Since TFDEA has at its core the widely used technique of DEA, TFDEA inherits the ability to provide many of the same rich results. One of the key results yielded by DEA is the identification of targets and efficient peers [233]. Specifically, DEA constitutes the frontier of a production possibility set (PPS) based on "best practice" DMUs. Within this framework, relative efficiency is determined by comparing the performance of each unit against that of a (virtual) target formed by efficient peers. A practical interpretation is that efficient peers can serve as role models which inefficient DMUs can emulate so that they may improve their performances. In other words, those benchmarks have a mix of inputoutput levels similar to that of DMUs being compared, which indicates that they are likely to operate in analogous environments and/or to favor similar operating practices [209].

The implementation of TFDEA relies on a series of benchmarking processes over time [223]. This is depicted in Fig. 9, assuming an output-oriented DEA model under variable returns to scale (VRS) [234]. The frontier year, $T$, is the point in time at which the analysis is conducted. Products $G, H$, and $I$ are identified to be the most competitive at time $T$ and therefore define the SOA frontier at time $T$. Products $A \sim F$, in contrast, were themselves SOA when they were first released but were superseded by subsequent products and hence are located below the frontier. Products $J$ and $K$ are future products, 
i.e., sets of specifications used as forecasting targets that are placed beyond the current SOA frontier.

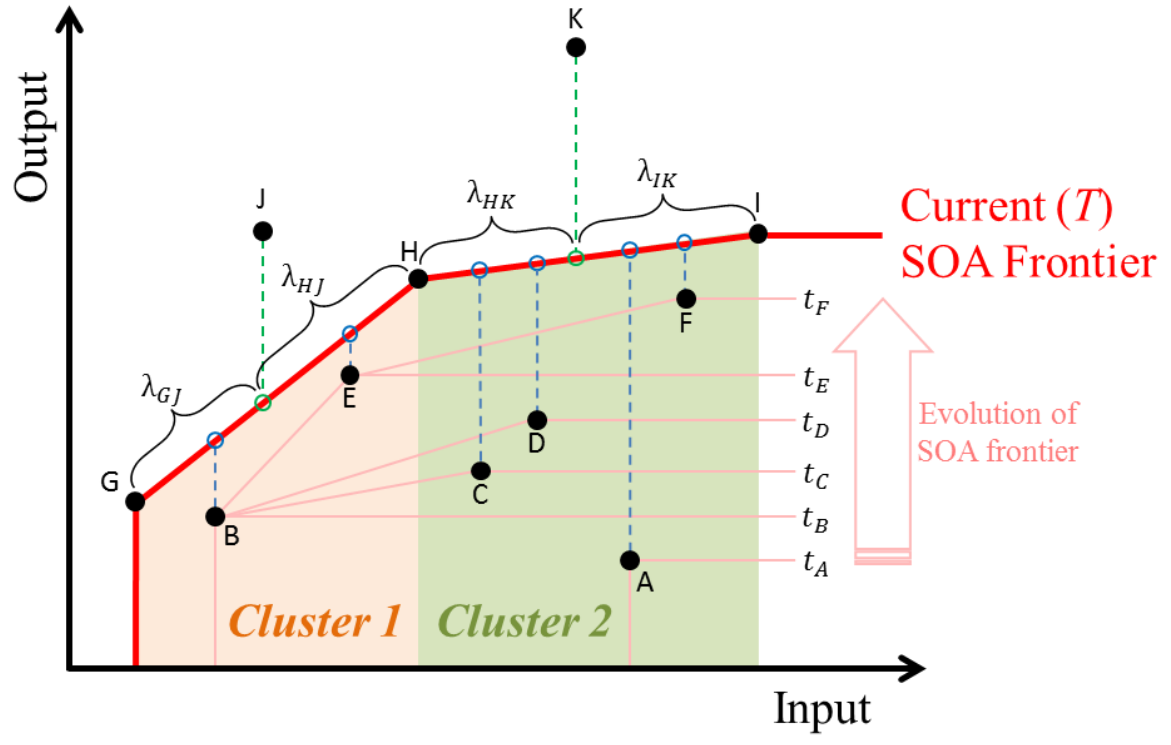

Figure 9 Evolution of the SOA frontier

The TFDEA process can be understood as three procedural stages. First, it iterates the DEA process to obtain efficiency scores of products both at the time of release and at the frontier year. Second, it estimates an RoC that represents how fast products have been replaced by the next generation products. In other words, the RoC indicates a potential growth rate of the SOA frontier in the future. Finally, the model makes a forecast of future products based on the average RoC.

The original TFDEA process simply aggregates RoCs from the past products and uses the average $\mathrm{RoC}$ to make a projection without taking technological segmentation into account. However, as previously discussed, DEA provides pragmatic information regarding benchmarks, which enables an identification of distinct product clusters. This 
information can be obtained either by reference sets in the envelopment model or by weighting schemes in the multiplier model.

For example, two different product clusters are identified in Fig. 9. The first cluster can be characterized by an optimized weighting scheme, that is, a facet connecting products $G$ and $H$. This can be interpreted that inefficient products from this cluster, namely $B$ and $E$, may have similar mixes of input-output levels such that a corresponding weighting scheme will show them in the best possible light. This can also be recognized as a reference set in the envelopment model since their performances are compared against virtual targets constituted by efficient peers, namely products $G$ and $H$.

In the same manner, the second cluster can represent another weighting scheme, that is, a facet connecting products $H$ and $I$. Even though the underlying products, $A, C, D$, and $F$, have less efficient input and output amounts, they must have similar ratios of the input-output levels that require the common weighting scheme to optimize their operations. The envelopment model, likewise, will constitute virtual products interpolated by products $\mathrm{H}$ and I for these inefficient products.

The idea of segmented $\mathrm{RoC}$ arises when there is a need to draw a distinction between each cluster; hence, the growth potential should be explained by local RoCs rather than a universal RoC. In our example, one may notice that cluster 2 has observed faster RoCs than cluster 1 . Specifically, products $B$ and $E$ did not show a large performance gap compared to the current SOA frontier even though the old product $B$, in particular, had stayed on the SOA frontier for a long time and only recently became superseded. This implies that the technological progress within cluster 1 has been neither fast nor frequent. In contrast, products pertinent to cluster 2 have shown successive 
replacements with substantial performance advancement over time. This may imply that more engineering effort has been invested in cluster 2-type products, which results in more frequent introductions of advanced products over time.

Once distinguishing clusters are identified with varying RoCs, it is readily possible to make a forecast using those local RoCs. For example, the estimated arrival of future product $J$ can be determined by measuring how far it is from the current SOA frontier and then extracting the root of that distance using local RoCs from cluster 1 given the fact that it is projected to the frontier facet of cluster 1 . In the same manner, the arrival of future product $K$ can be estimated using local RoCs from cluster 2. One may expect that if both products were achievable with the same amount of engineering advances, the arrival of product $K$ might be earlier than that of product $J$ since faster progress is expected from cluster 2-type products. In other words, requiring the same amount of time to reach the technological level of product $J$ would entail significant development risk.

Figure 10 depicts how the local $\mathrm{RoC}$ and individualized $\mathrm{RoC}$ can be obtained. Product $L$ had been located on the SOA frontier in the past but later became obsolete by the current SOA frontier formed by new competitive products: $M, N$, and $O$. As aforementioned, the fact that product $L$ is compared to its virtual target, i.e., $L^{\prime}$, constituted by its peers: $M, N$, and $O$ indicates that product $L$ may have a similar mix of input-output levels with those peers although the absolute level of attributes may vary, which can classify them as homogeneous products. Hence, the technological advancement, namely the performance gap between $L$ and $L^{\prime}$ during a given time period, can be represented by the peers as a form of local RoC. Consequently, each local RoC 
indicates a growth potential for adjacent frontier facets based on the technological advancement observed from the related past products.

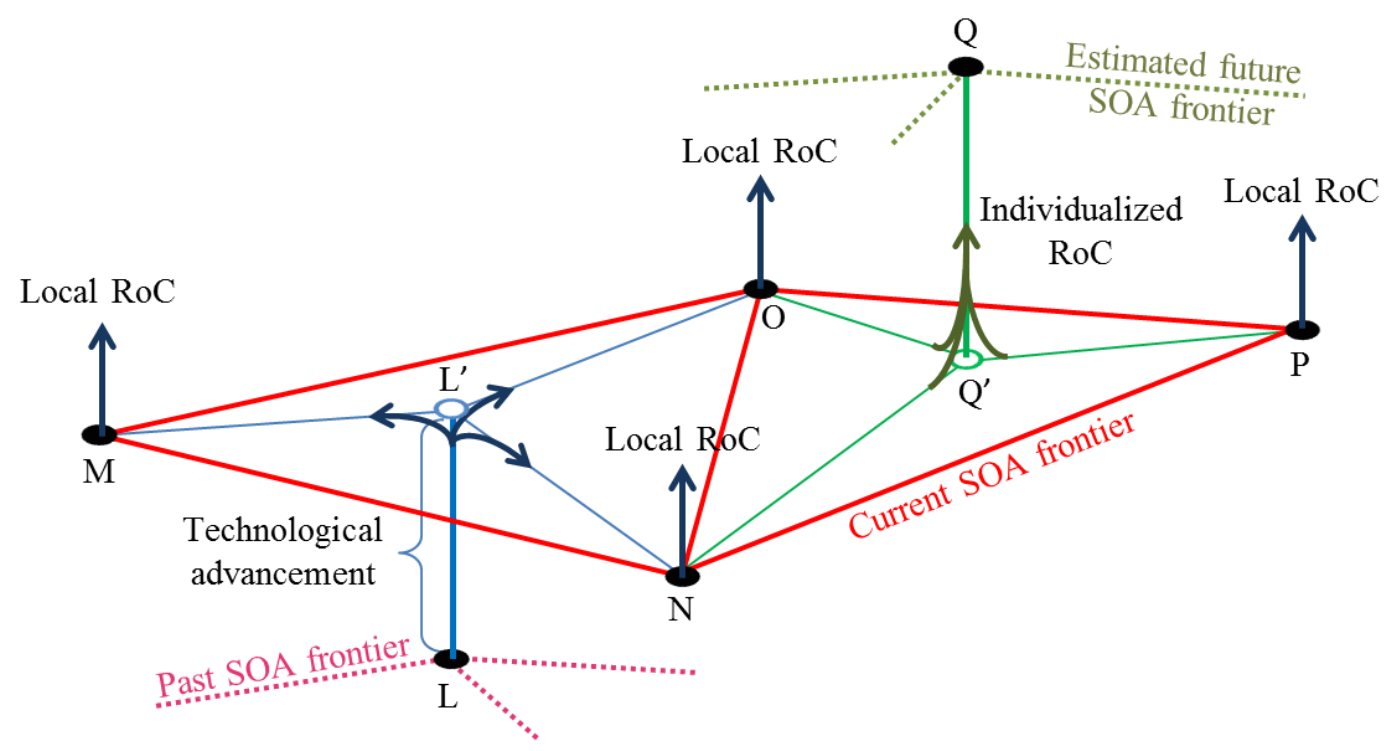

Figure 10 Illustration of segmented rate of change

Once the local RoC of current SOA products are obtained, it is straightforward to compute the individualized RoC for the new product concepts. Suppose product developers came up with a product concept $Q$. Note that by definition, a "better" product would be located beyond the current SOA frontier as superseded products are located below, namely enveloped by, the current SOA frontier. It is seen that the virtual target of $Q$, i.e., $Q^{\prime}$, is subject to the frontier facet constituted by current SOA products $N, O$, and $P$. Thus, the individualized RoC of $Q$ can be obtained by combining local RoCs with the reference information: how close $Q^{\prime}$ is from $N, O$, and $P$ respectively. It should be noted here that technological advancement observed from the product $L$ may have affected the individualized RoC of $Q$ as SOA product $N$ and $O$ are involved in both sides of the facets 
by having intermediate technological characteristics. This information can give insight to the product developers not only about who the major players in a target market are but also about how competitive the proposed design concept is. In other words, this can provide a diagnostic if the proposed design concept is aggressive or conservative in terms of scheduled delivery to the market considering the current rate of technological progress expected in a target segment. One can also utilize this information to estimate the arrival of a competitor's design targets as a post product launch strategy of their own.

As we increase the dimension, i.e., the number of structural characteristics and/or functional features of the product being considered, the problem can be better handled by algebraic formulation than graphical analysis. The underlying formulation is introduced in section 3.2. 


\subsubsection{INFEASIBLE FORECASTING TARGET ${ }^{3}$}

It has been observed in past super-efficiency DEA applications that the infeasibility problem occurs when variable returns to scale (VRS) was assumed [27]. In theory, this is also a problem for an input-oriented decreasing returns to scale (DRS) model and outputoriented increasing returns to scale (IRS) model. This problem results in failure to make a forecast in TFDEA since the model is unable to measure the super-efficiency, $\theta_{0}^{t}\left(x_{0}^{T>t}, y_{0}^{T>t}\right)$, i.e., superiority of specified technical capabilities from the current frontier. Note that the constant returns to scale (CRS) DEA model is also susceptible to this problem when a zero value is included in an input variable [209]. However, this is rare in TFDEA applications since it indicates heterogeneous technologies.

Figure 11 depicts possible occasions in which infeasible super-efficiency occurs under VRS. It is readily seen that target $E$ and $F$ are subject to infeasibility from the current frontier in the input-oriented (IO) model and output-oriented (OO) model respectively, whereas target $D$ is infeasible in both orientations. Therefore, the arrivals of those targets in corresponding orientation from the current frontier are unable to be computed using the traditional TFDEA model.

${ }^{3}$ This section is adapted from a paper accepted in International Transactions in Operational Research [313] 


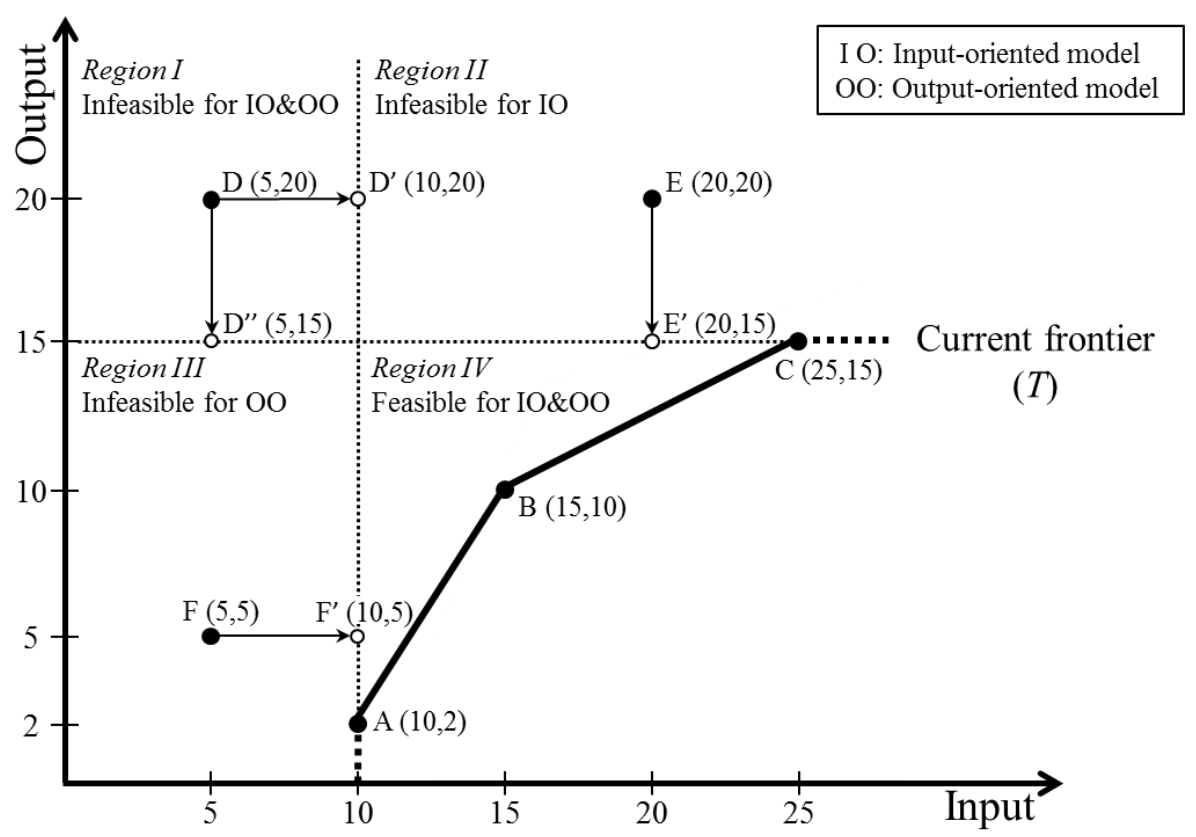

Figure 11 Regions of infeasible super-efficiency under VRS DEA model

Alternate measures have been developed to deal with the infeasible super-efficiency problem. Lovell and Rouse suggested employing a user-defined scaling factor to make the VRS super-efficiency model feasible [235]. Cook et al. developed a radial measure of super-efficiency with respect to both input and output direction so that one can derive the minimum change needed to project a DMU to a non-extreme position, and the other can reflect the radial distance of that shifted DMU from the frontier formed by the remaining DMUs [236]. In a similar vein, Lee et al. proposed a slack based superefficiency model that can consider both input savings and output surplus in cases where infeasibility occurs [237]. Lee and Zhu further extended this model to deal with the infeasibility problem caused by zero input values [238].

In this study, Cook et al.'s alternate super-efficiency measure is adopted for two main reasons: a) it returns bi-oriented $L_{1}$ distances for infeasible targets and hence it 
secures the existing RoC calculation; b) it returns the same radial distance as the standard super-efficiency measure [239] when the target is feasible.

Cook et al. [236] defined the term "extremity" to indicate a minimum radial movement in either direction needed for a DMU to reach a non-extreme position. For example, in the input-oriented model, target $E$ will have an extremity of $0.75(=15 / 20)$, to bring it down to the closest feasible point, i.e., $E^{\prime}(20,15)$. The radial input augmentation is then applied, i.e., $1.25(=25 / 20)$, from this shifted point $E^{\prime}$ to the peer unit $C$. Consequently, the input-oriented super-efficiency of target $E$ from the current frontier can be defined as $2.583(=1.25+1 / 0.75)$. In a similar sense, the output-oriented superefficiency of target $\mathrm{F}$ from the current frontier is $4.5(=2+1 / 0.4)$, and target $D$ has 6.333 $(=5+1 / 0.75)$ and $12(=2+1 / 0.1)$ from the input-oriented model and output-oriented model respectively.

Once the super-efficiency score of each forecasting target from the current frontier is obtained, RoCs can be applied to the estimation of those target technologies' arrivals. Note that targets that contain extremities in their super-efficiency scores require RoCs from both orientations. That is, the time period for the extremity can be estimated by the RoC from the opposite orientation model. In the case of target $F$, for example, the outputoriented TFDEA model should be able to compute how long it will take to reduce the input from 10 to 5 based on the RoC that one would obtain from the input-oriented model as well as to augment the output from 2 to 5 based on the output-oriented RoC. This indicates that performing TFDEA in both orientations is required to deal with the infeasible forecasting targets. 


\subsection{FORMUlation}

I now turn to the TFDEA formulation incorporating the proposed approach under VRS. The entire process can be divided into four separate stages.

The first stage iterates efficiency measurement in a time series manner so that the evolution of the SOA frontier can be monitored. As mentioned above, it is required to obtain RoCs in both orientations to make a forecast for targets containing extremities. Therefore, this stage computes radial efficiencies: $\phi_{k}^{h \in\{R, C\}}$ from the output-oriented model shown by (12)-(18) and $\theta_{k}^{h \in\{R, C\}}$ from the input-orientation model shown by (19)(25). Note that the model can be formulated as a single large LP, it may also be formulated and solved as a series of equivalent, smaller LP models for the time of release (R) and models for the current frontier time (C) depending on the implementation algorithm. Specifically, $x_{i j}$ represents the $i$ th input and $y_{r j}$ represents the $r$ th output for each technology $j=1, \ldots, n$, and $j=k$ identifies the technology to be evaluated.

The objective functions for each orientation, (12) and (19), incorporate minimizing effective dates as well to ensure reproducible outcomes from possible alternate optimal solutions by distinguishing between Pareto-efficient technologies ${ }^{4}$ [240], [241].

Constraints (15), (16), (22), and (23) limit the reference sets so that two types of efficiencies, one at the time of release $(R)$ and the other at the current frontier time $(C)$ in

\footnotetext{
${ }^{4}$ Unlike the iso-time frontier from parametric frontier models, the technology frontier constructed by TFDEA typically consists of multiple vintages of SOA technologies. This allows the model to specify the individual timing, i.e., effective date, of any points on the frontier according to the corresponding tradeoff surface. Therefore, the issue of alternate optimal solutions occurs either due to weakly efficient technology or to an efficient but not an extreme technology, namely $F$ type or $E$ ' type in Charnes et al.'s classification [314]. Both cases can be dealt with by introducing the secondary objective to choose the reference technology presenting either in the farthest time horizon, i.e., maximum sum of effective date, or in the closest time horizon, i.e., minimum sum of effective date. Note that depending on the application area, slack maximization may be preferred to prevent weakly efficient technologies from setting the effective date. Further discussion can be found in [240], [315].
} 
which the forecast is conducted, are obtained. That is, $\phi_{k}^{R}$ and $\theta_{k}^{R}$ each measure the amount by which technology $k$ is surpassed by the technologies available at the time of release since constraint (15) and (22) allow the reference set of technology $k$ to only include technologies that had been released up to $t_{k}$. Similarly, $\phi_{k}^{C}$ and $\theta_{k}^{C}$ can be interpreted as how superior technology $k$ is compared to the current SOA frontier by constraint (16) and (23).

Note that the "current time" is defined as a fixed time $T$, which can be either the most recent time in the dataset or a certain point in time as a forecasting origin when the time series hold-out sampling is performed. The variable $\lambda_{j k}^{h}$ describes how much of technology $j$ is used in setting a target of performance for technology $k$.

Note that in the case of the VRS model, constraint (17) and (24) would allow replacing the denominator in the second term of (12) and (19) with a 1 , making the objective functions linear. Here, it is imperative that the value of a non-Archimedean infinitesimal, $\varepsilon$, not be implemented as a finite approximation to avoid inaccuracies and erroneous results [241]. Instead, the actual implementation is to use a two-stage preemptive linear programming to first identify the radial efficiency and then to either maximize (or minimize) effective dates or to maximize the slacks according to the need. 


$$
\begin{aligned}
& \max \sum_{h} \sum_{k=1}^{n}\left[\phi_{k}^{h}-\varepsilon\left(\frac{\sum_{j=1}^{n} \lambda_{j k}^{h} \cdot t_{j}}{\sum_{j=1}^{n} \lambda_{j k}^{h}}\right)\right] \\
& \text { s.t. } \sum_{j=1}^{n} \lambda_{j k}^{h} \cdot y_{r j} \geq \phi_{k}^{h} \cdot y_{r k}, \quad \forall r, k, h \in\{R, C\} \\
& \sum_{j=1}^{n} \lambda_{j k}^{h} \cdot x_{i j} \leq x_{i k}, \quad \forall i, k, h \in\{R, C\} \\
& \lambda_{j k}^{R}=0, \quad \forall(j, k) \mid t_{j}>t_{k} \\
& \lambda_{j k}^{C}=0, \quad \forall(j, k) \mid t_{j}>T \\
& \sum_{j=1}^{n} \lambda_{j k}^{h}=1, \quad \forall k, h \in\{R, C\} \\
& \lambda_{j k}^{h} \geq 0, \quad \forall j, k, h \in\{R, C\} \\
& \min \sum_{h} \sum_{k=1}^{n}\left[\theta_{k}^{h}+\varepsilon\left(\frac{\sum_{j=1}^{n} \mu_{j k}^{h} \cdot t_{j}}{\sum_{j=1}^{n} \mu_{j k}^{h}}\right)\right] \\
& \text { s.t. } \sum_{j=1}^{n} \mu_{j k}^{h} \cdot y_{r j} \geq y_{r k}, \quad \forall r, k, h \in\{R, C\} \\
& \sum_{j=1}^{n} \mu_{j k}^{h} \cdot x_{i j} \leq \theta_{k}^{h} \cdot x_{i k}, \quad \forall i, k, h \in\{R, C\} \\
& \mu_{j k}^{R}=0, \quad \forall(j, k) \mid t_{j}>t_{k} \\
& \mu_{j k}^{C}=0, \quad \forall(j, k) \mid t_{j}>T \\
& \sum_{j=1}^{n} \mu_{j k}^{h}=1, \quad \forall k, h \in\{R, C\} \\
& \mu_{j k}^{h} \geq 0, \quad \forall j, k, h \in\{R, C\}
\end{aligned}
$$


The non-VRS models such as non-decreasing returns to scale (NDRS), nonincreasing returns to scale (NIRS), or CRS would render the objective function, (12) and (19), to no longer be linear as the denominator is not constrained to be equal to 1 . For computational purposes, the same general secondary goal of minimizing effective years can also be approximated by subtracting the sum of reference vectors, $\sum_{j=1}^{n} \lambda_{j k}^{h}$ and $\sum_{j=1}^{n} \mu_{j k}^{h}$ respectively, in the objective function as seen in (26) and (27). While this substitution is not technically a numerical approximation, it is generally consistent with minimizing effective year and has the advantage of remaining linear [240].

$$
\begin{array}{r}
\max \sum_{h} \sum_{k=1}^{n}\left[\phi_{k}^{h}-\varepsilon\left(\sum_{j=1}^{n} \lambda_{j k}^{h} \cdot t_{j}-\sum_{j=1}^{n} \lambda_{j k}^{h}\right)\right] \\
\min \sum_{h} \sum_{k=1}^{n}\left[\theta_{k}^{h}+\varepsilon\left(\sum_{j=1}^{n} \mu_{j k}^{h} \cdot t_{j}-\sum_{j=1}^{n} \mu_{j k}^{h}\right)\right]
\end{array}
$$

The second stage, shown by (28)-(31), calculates the RoCs from each orientation, $\gamma_{k}^{C}\left(\beta_{k}^{C}\right)$, by taking all technologies that were efficient at the time of release, $\phi_{k}^{R^{*}}=1$ $\left(\theta_{k}^{R^{*}}=1\right)$, but later superseded by new technologies at the current frontier time, $\phi_{k}^{C^{*}}>1$ $\left(\theta_{k}^{C^{*}}<1\right)$. Having calculated RoCs of past technologies in (28) and (30), the idea of segmented RoC can then be implemented by taking the weighted average of RoC for each technology on the current SOA frontier. This leads to the calculation of local RoCs in (29) and (31), where $\delta_{j}^{C}\left(\beta_{k}^{C}\right)$ represents the local RoC driven by technology $j$ at current time $T$. Note that technology $j$ has an efficiency score of 1 at the current frontier; in other 
words, it is one of the SOAs that constitutes the frontier onto which future technologies are to be projected. The numerator of (29) and (31) indicates the weighted sum of RoCs from past technologies that have set technology $j$ as a (or one of) benchmark(s). The denominator indicates the accumulated contribution of technology $j$ to the evolution of the SOA frontier. Consequently, $\delta_{j}^{C}\left(\beta_{k}^{C}\right)$ represents the local RoC that only counts RoCs in which SOA technology $j$ has been used as a benchmark ${ }^{5}$.

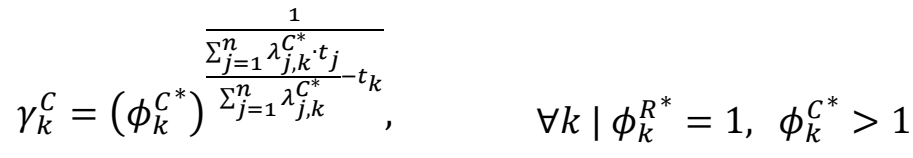

$$
\begin{aligned}
& \delta_{j}^{C}=\frac{\sum_{k=1}^{n} \lambda_{j, k}^{C^{*}} \cdot \gamma_{k}^{C}}{\sum_{k=1, \gamma_{k}^{C}>0}^{n} \lambda_{j, k}^{C^{*},}} \quad \forall j \mid \phi_{j}^{C^{*}}=1 \\
& \beta_{k}^{C}=\left(\frac{1}{\theta_{k}^{C^{*}}}\right)^{\frac{1}{\sum_{j=1}^{n} \mu_{j k}^{C_{k}^{*} \cdot t_{j}}} \mu_{j k}^{C_{j}^{n}}}, \quad \forall k \mid \theta_{k}^{R^{*}}=1, \theta_{k}^{C^{*}}<1 \\
& \zeta_{j}^{C}=\frac{\sum_{k=1}^{n} \mu_{j k}^{C^{*}} \cdot \beta_{k}^{C}}{\sum_{k=1, \beta_{k}^{C}>0}^{n} \mu_{j k}^{C^{*}}}, \quad \forall j \mid \theta_{j}^{C^{*}}=1
\end{aligned}
$$

\footnotetext{
${ }^{5}$ A special case [316] was observed in which the RoC for one product, i.e., $\gamma_{k}^{C}\left(\beta_{k}^{C}\right)$, exceeded 10.0 due to the short time period between the effective date and the actual release date. An RoC of 10.0 indicates that the technology is advancing at a rate of $1000 \%$ per time period (day, month, year). This greatly exceeds even the rapidly moving portions of the computer industry such as microprocessors and therefore is considered an unreliable estimate of RoC. The current implementation assumes the maximum acceptable $\mathrm{RoC}$ to be 10.0 and hence drops those having RoCs greater than this limit from the local RoC calculation. Exploring this further is a topic for future work.
} 
The third stage solves super-efficiency models for the forecasting targets of future products. Since the purpose of this stage is to measure the super-efficiency of each forecasting target from the current frontier, the reference set is confined to the current SOA technologies by (35), (36), (41), and (42). $M$ is a user-defined large positive number to give a preemptive priority to the identification of a minimum radial shift of inputs (or outputs) to render the model feasible. In the output-oriented model, shown by (32)-(37), radial output reduction and extremity are obtained as $1-\chi_{k}^{O}$ and $1+\psi_{k}^{O}$, respectively. Likewise, in the input-oriented model, shown by (38)-(43), radial input augmentation and extremity are defined as $1+\tau_{k}^{O}$ and $1-\rho_{k}^{O}$, respectively.

$$
\begin{array}{ll}
\min \sum_{k \mid t_{k}>T}\left(\chi_{k}+M \cdot \psi_{k}\right) & \\
\text { s.t. } \sum_{j=1}^{n} \lambda_{j k}^{C} \cdot x_{i j} \leq\left(1+\psi_{k}\right) \cdot x_{i k}, & \forall i, k \\
\sum_{j=1}^{n} \lambda_{j k}^{C} \cdot y_{r j} \geq\left(1-\chi_{k}\right) \cdot y_{r k}, & \forall r, k \\
\sum_{j=1}^{n} \lambda_{j k}^{C}=1, & \forall k \mid \phi_{k}^{C^{*}}=1 \\
\lambda_{j k}^{C}=0, & \forall(j, k) \mid t_{j}>T \\
\psi_{k}, \lambda_{j k}^{C} \geq 0, & \forall j, k
\end{array}
$$




$$
\begin{array}{ll}
\min & \sum_{k \mid t_{k}>T}\left(\tau_{k}+M \cdot \rho_{k}\right) \\
\text { s.t. } \sum_{j=1}^{n} \mu_{j k}^{C} \cdot x_{i j} \leq\left(1+\tau_{k}\right) \cdot x_{i k}, & \forall i, k \\
\sum_{j=1}^{n} \mu_{j k}^{C} \cdot y_{r j} \geq\left(1-\rho_{k}\right) \cdot y_{r k}, & \forall r, k \\
\sum_{j=1}^{n} \mu_{j k}^{C}=1, & \forall k \mid \theta_{k}^{C *}=1 \\
\mu_{j k}^{C}=0, & \forall(j, k) \mid t_{j}>T \\
\rho_{k}, \mu_{j k}^{C} \geq 0, & \forall j, k
\end{array}
$$

The last stage makes a forecast of the arrival of future technologies. The individualized $\mathrm{RoC}$ for each forecasting target $k$ can be computed by combining the local RoCs of SOA technology $j$ that constitutes the frontier facet onto which technology $k$ is being projected. That is, in the case of the output-oriented (input-oriented) model, the estimated elapsed time for the extremity, if any, is computed using the individualized RoC from the input-oriented (output-oriented) model. For target $F$ in Fig. 11, for example, the time span for the extremity, namely distance from $F$ to $F^{\prime}, 2(=10 / 5)$, from the outputoriented model is estimated by individualized RoC combined with input-oriented local RoCs of its peers: $A$ and $B$. In addition, the time span for radial output reduction, namely, distance from $F^{\prime}$ to $A, 0.4(=2 / 5)$, is estimated by individualized RoC from its outputoriented peer, $A$. Consequently, the forecasted arrival time of $F$ is obtained by the sum of those estimated elapsed times and the reference time of the current frontier. Likewise, the forecasted arrival time of $D$ under the output-oriented (input-oriented) model is obtained 
by the sum of the estimated time span for the distance from itself to a radially shifted point, i.e., $D^{\prime}\left(D^{\prime \prime}\right)$, using input-oriented (output-oriented) local RoC of $C(A)$ and estimated time span for the distance from the shifted point to its peer, $A(C)$, using corresponding output-oriented (input-oriented) local RoC.

$$
\begin{aligned}
t_{k}^{\text {forecast_oo }} & =\frac{\ln \left(\frac{1}{1-\chi_{k}^{O}}\right)}{\ln \left(\frac{\sum_{j=1}^{n} \lambda_{j, k}^{C^{O}} \cdot \delta_{j}^{C}}{\sum_{j=1}^{n} \lambda_{j, k}^{C^{O}}}\right)}+\frac{\ln \left(1+\psi_{k}^{O}\right)}{\ln \left(\frac{\sum_{j=1}^{n} \mu_{j, k}^{C^{O}} \cdot \zeta_{j}^{C}}{\sum_{j=1}^{n} \mu_{j, k}^{C O}}\right)}+\frac{\sum_{j=1}^{n} \lambda_{j, k}^{C^{O}} \cdot t_{j}}{\sum_{j=1}^{n} \lambda_{j, k}^{C^{O}}}, \quad \forall k \mid t_{k}>T \\
t_{k}^{\text {forecast_IO }} & =\frac{\ln \left(1+\tau_{k}^{O}\right)}{\ln \left(\frac{\sum_{j=1}^{n} \mu_{j, k}^{C^{O}} \cdot \zeta_{j}^{C}}{\sum_{j=1}^{n} \mu_{j, k}^{C}}\right)}+\frac{\ln \left(\frac{1}{1-\rho_{k}^{O}}\right)}{\ln \left(\frac{\sum_{j=1}^{n} \lambda_{j, k}^{C^{O}} \cdot \delta_{j}^{C}}{\sum_{j=1}^{n} \lambda_{j, k}^{C^{O}}}\right)}+\frac{\sum_{j=1}^{n} \mu_{j, k}^{C^{O}} \cdot t_{j}}{\sum_{j=1}^{n} \mu_{j, k}^{C^{O}}}, \quad \forall k \mid t_{k}>T
\end{aligned}
$$

Equations (32) and (33) yield the same solution, when the original TFDEA model is feasible, and provide results with consistent interpretation when the original TFDEA model is infeasible. The following proofs of theorem (32) and (33) guarantee that the proposed TFDEA extension returns a feasible and a finite solution.

Theorem 1 (44) always yields a finite forecast.

Proof If the target $k$ is feasible from the original super-efficiency model in [239], this means that $\psi_{k}^{O}=0$ by theorem 3 in [236]. This reduces (44) to the traditional outputoriented TFDEA model in [223]. If the target $k$ is infeasible from the original superefficiency model in [239], this means that $\psi_{k}^{O}>0$ by theorem 3 in [236]. In both cases, 
$1 /\left(1-\chi_{k}^{O}\right)>0$ by theorem 4 in [236]. Therefore, $t_{k}^{\text {forecast_oo }}$ is always feasible. This completes the proof.

Theorem 2 (45) always yields a finite forecast.

Proof If the target $k$ is feasible from the original super-efficiency model in [239], this means that $\rho_{k}^{O}=0$ by theorem 1 in [236]. This reduces (45) to the traditional inputoriented TFDEA model in [223]. If the target $k$ is infeasible from the original superefficiency model in [239], this means that $\rho_{k}^{O}>0$ by theorem 1 in [236]. In both cases, $1+\tau_{k}^{O}>0$ by theorem 2 in [236]. Therefore $t_{k}^{\text {forecast_IO }}$ is always feasible. This completes the proof. 


\subsection{ILluSTRATIVE EXAMPLE}

To illustrate the use of the proposed approach, this section revisits the TFDEA application to the LCD panel industry [232]. The dataset includes 389 models from 20 manufacturers that have been introduced to the market from 1997 to 2012. For the sake of showing the comparative results, a hold-out sample test is conducted by dividing the dataset into two parts, a training set (1997 2007) and testing set (2008 2012), to validate the forecasting model using two input parameters: bezel size and weight and three output parameters: screen size, resolution, and contrast ratio.

Table 13 summarizes the forecast results of those products which were infeasible in the traditional input-oriented VRS TFDEA model. That is, infeasibilities rendered the traditional TFDEA model unable to make a forecast for the listed 31 targets out of 95 future technologies from the forecasting origin of 2007. This is shown by the value of extremities in the fourth column indicating the necessity of output reductions to be able to compute the radial input-oriented super-efficiency scores shown in the fifth column.

As previously discussed, an infeasible target in the input-oriented TFDEA may occur when the output levels of the target technology are unprecedented in the past. In other words, there is no way a radial expansion in the inputs of the target technology, keeping outputs fixed, can cross the current SOA frontier. Therefore, one can interpret this large portion of infeasible targets as an indicator of active technological advancements, particularly in terms of output parameters that post-2007 LCD panels achieved. This is actually in line with the dynamics of the flat panel industry in which the manufacturers kept investing their engineering efforts toward a higher performance standard such as ultra-high definition (UHD) with lifelike contrast ratio. 
Table 13 Forecast results for infeasible targets

\begin{tabular}{|c|c|c|c|c|c|c|c|c|}
\hline $\begin{array}{l}\text { DMU } \\
(k)\end{array}$ & $\begin{array}{l}\text { LCD } \\
\text { panel name }\end{array}$ & $\begin{array}{c}\text { Actual } \\
\text { year of release } \\
\left(t_{k}\right)\end{array}$ & $\begin{array}{c}\text { Extremity } \\
\left(1-\rho_{k}^{O}\right)\end{array}$ & $\begin{array}{l}\text { Radial distance } \\
\quad\left(1+\tau_{k}^{O}\right)\end{array}$ & $\begin{array}{c}\text { Individualized } \\
\text { output-oriented RoC } \\
\left(\frac{\sum_{j=1}^{n} \lambda_{j, k}^{C^{o}} \cdot \delta_{j}^{C}}{\sum_{j=1}^{n} \lambda_{j, k}^{C^{O}}}\right)\end{array}$ & $\begin{array}{c}\text { Individualized } \\
\text { input-oriented RoC } \\
\left(\frac{\sum_{j=1}^{n} \mu_{j, k}^{C^{o}} \cdot \zeta_{j}^{C}}{\sum_{j=1}^{n} \mu_{j, k}^{C^{O}}}\right)\end{array}$ & $\begin{array}{l}\text { Effective date } \\
\left(\frac{\sum_{j=1}^{n} \mu_{j, k}^{C^{o}} \cdot t_{j}}{\sum_{j=1}^{n} \mu_{j, k}^{C^{O}}}\right)\end{array}$ & $\begin{array}{c}\text { Forecasted } \\
\text { time of release } \\
\left(t_{k}^{\text {forecast_IO }}\right)\end{array}$ \\
\hline 165 & T520HW01 V0 & 2008 & 0.9351 & 1.6236 & 1.1778 & 1.1972 & 2006.72 & 2009.82 \\
\hline 166 & V562D1-L04 & 2008 & 0.9632 & 1.0035 & 1.1876 & 1.6580 & 2007.00 & 2007.16 \\
\hline 212 & V460H1-LH7 & 2009 & 0.8183 & 1.3059 & 1.1932 & 1.1879 & 2006.86 & 2009.53 \\
\hline 218 & LTA550HF02 & 2009 & 0.9151 & 2.0317 & 1.1708 & 1.1986 & 2006.70 & 2011.16 \\
\hline 248 & V400H1-L08 & 2009 & 0.8508 & 1.2724 & 1.1984 & 1.1849 & 2006.90 & 2009.21 \\
\hline 265 & LK460D3LA63 & 2010 & 0.9778 & 2.2312 & 1.1752 & 1.1941 & 2006.77 & 2011.43 \\
\hline 266 & LTA460HM03 & 2010 & 0.9778 & 1.7685 & 1.1826 & 1.1941 & 2006.77 & 2010.11 \\
\hline 268 & LTA460HQ05 & 2010 & 0.9778 & 2.0760 & 1.1824 & 1.1941 & 2006.77 & 2011.02 \\
\hline 271 & P460HW03 V0 & 2010 & 0.9778 & 2.0760 & 1.1824 & 1.1941 & 2006.77 & 2011.02 \\
\hline 273 & V460H1-L11 & 2010 & 0.8183 & 1.2929 & 1.1932 & 1.1879 & 2006.86 & 2009.48 \\
\hline 274 & V460H1-LH9 & 2010 & 0.8653 & 1.4340 & 1.1900 & 1.1898 & 2006.83 & 2009.73 \\
\hline 276 & LK601R3LA19 & 2010 & 0.6737 & 1.4558 & 1.1609 & 1.4553 & 2007.00 & 2010.27 \\
\hline 277 & LTA550HJ06 & 2010 & 0.8159 & 1.7228 & 1.1738 & 1.1940 & 2006.77 & 2011.09 \\
\hline 282 & P546HW02 V0 & 2010 & 0.9151 & 2.3603 & 1.1415 & 1.1986 & 2006.70 & 2012.11 \\
\hline 283 & P645HW03 V0 & 2010 & 0.9674 & 1.8781 & 1.1413 & 1.2088 & 2006.56 & 2010.13 \\
\hline 284 & P650HVN02.2 & 2010 & 0.9674 & 1.7607 & 1.1603 & 1.2088 & 2006.56 & 2009.75 \\
\hline 293 & R300M1-L01 & 2010 & 0.7910 & 2.7735 & 1.1156 & 1.6838 & 2007.00 & 2009.52 \\
\hline 298 & LTF320HF01 & 2010 & 0.9590 & 1.1092 & 1.2043 & 1.1817 & 2006.95 & 2007.79 \\
\hline 305 & V315H3-L01 & 2010 & 0.9590 & 1.3354 & 1.2039 & 1.1817 & 2006.95 & 2008.91 \\
\hline 321 & T400HW03 V3 & 2010 & 0.9018 & 1.2863 & 1.1955 & 1.1866 & 2006.88 & 2008.92 \\
\hline 325 & V420H2-LE1 & 2010 & 0.8893 & 1.9215 & 1.1832 & 1.1877 & 2006.86 & 2011.35 \\
\hline 330 & V370H4-L01 & 2010 & 0.9211 & 1.3982 & 1.1982 & 1.1849 & 2006.90 & 2009.33 \\
\hline 331 & V400H1-L10 & 2010 & 0.9018 & 1.4399 & 1.1952 & 1.1866 & 2006.88 & 2009.58 \\
\hline 336 & LTA460HN01-W & 2011 & 0.9778 & 1.9895 & 1.1825 & 1.1941 & 2006.77 & 2010.78 \\
\hline 341 & V500HK1-LS5 & 2011 & 0.9489 & 2.5756 & 1.1354 & 1.1962 & 2006.74 & 2012.43 \\
\hline 344 & BR650D15 & 2011 & 0.8571 & 1.3431 & 1.1650 & 1.2028 & 2006.64 & 2009.24 \\
\hline 349 & LK600D3LB14 & 2011 & 0.6012 & 1.5420 & 1.1686 & 1.1866 & 2006.88 & 2012.67 \\
\hline 350 & LK695D3LA08 & 2011 & 0.8268 & 1.8865 & 1.1446 & 1.2050 & 2006.61 & 2011.42 \\
\hline 353 & LTI700HA01 & 2011 & 0.9289 & 1.6223 & 1.1510 & 1.2110 & 2006.52 & 2009.56 \\
\hline 355 & T706DB01 V0 & 2011 & 0.9060 & 2.8878 & 1.1286 & 1.2345 & 2006.56 & 2012.41 \\
\hline 357 & V546H1-LS1 & 2011 & 0.8159 & 2.0305 & 1.1688 & 1.1940 & 2006.77 & 2012.06 \\
\hline
\end{tabular}


The sixth and seventh columns both show the individualized output-oriented RoC and input-oriented RoC. The time span required for output reduction, i.e., extremity, was therefore obtained using corresponding individualized output-oriented RoC. Likewise, the time span required for input augmentation, i.e., radial distance, was computed using the corresponding individualized input-oriented RoC.

The last column shows the forecasted year of release considering the superiority of each target technology compared to the 2007 SOA frontier. That is, the forecasted year of release was obtained by the sum of the optimal starting point of the forecast, i.e., effective date shown in the eighth column, and the estimated elapsed times for extremity and radial distance.

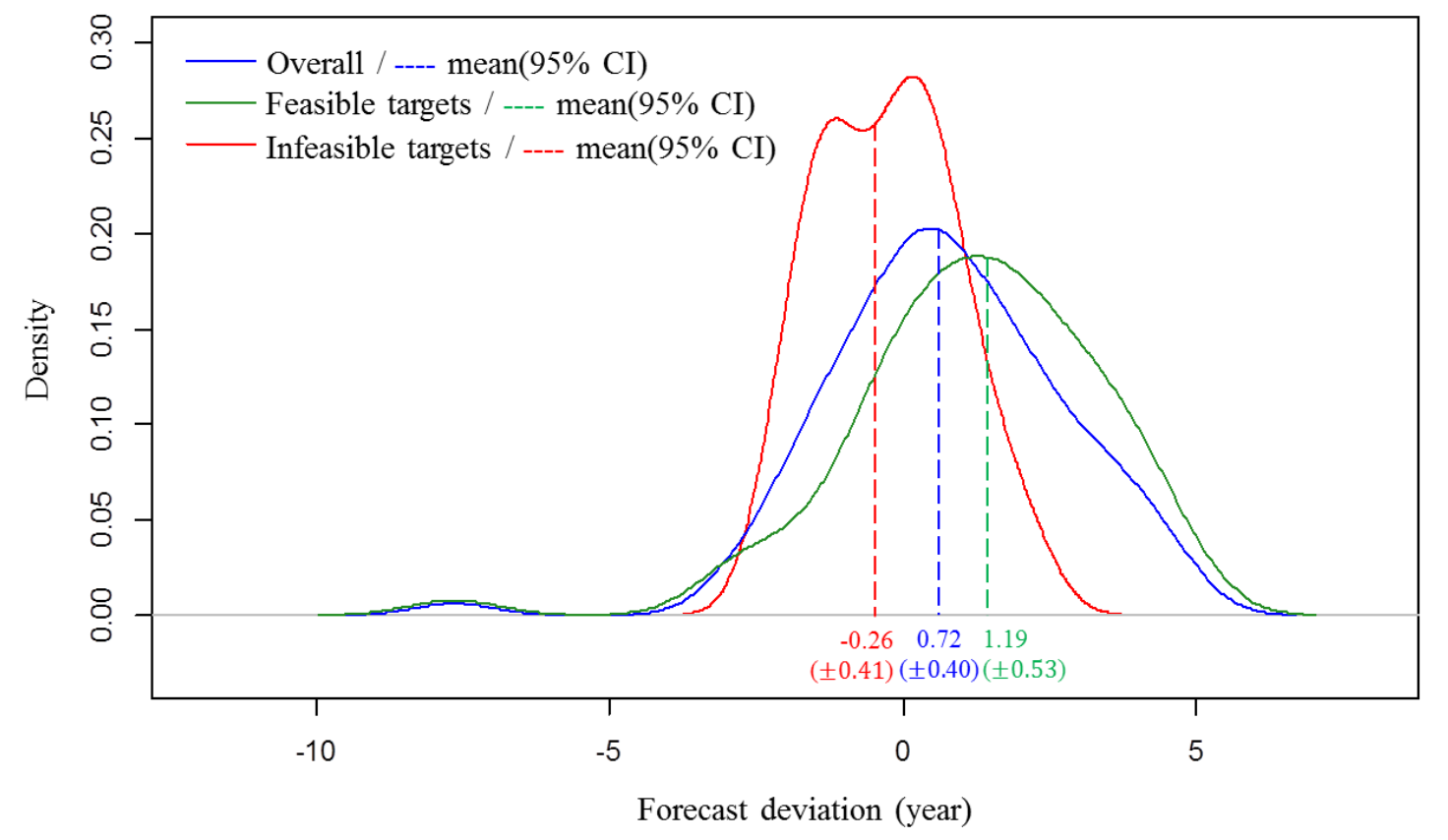

Figure 12 Forecast deviation distributions 
The accuracy of the proposed model can be readily shown by comparing those forecasted years with actual years of release. The deviation statistics contain this information. As seen from Fig. 12, forecast deviation distribution of 31 infeasible targets has a mean of -0.26 years with \pm 0.41 in a $95 \%$ confidence interval (CI). This is more accurate than a forecast of 64 feasible targets, i.e., mean deviation of +1.19 years $( \pm 0.53)$, which could improve the overall forecasting performance of a mean deviation of +0.72 years $( \pm 0.40)$. Note that the proposed model yielded the forecast results equivalent to that of a traditional TFDEA model for feasible targets. Consequently, it is shown that the proposed model could make a reasonable forecast for formerly infeasible targets as well as a consistent forecast for feasible targets. 


\section{VALIDATING THROUGH CASE STUDIES}

In this chapter, the proposed approach is applied to actual case studies. This is organized in two sections. The first section focuses on ex post analysis, which revisits the past applications to show how this approach can improve the forecasting accuracy using a hold-out sample technique. In particular, the case study of the commercial airplane industry is described in detail to fully explain the use of the proposed approach. To further validate the utility of the proposed approach, six past datasets are revisited and the comparative results are provided in comparison to the traditional approach.

The second section focuses on ex ante analysis, which addresses how the proposed approach can be used to solve the actual forecasting problems in the supercomputer industry. Specifically, the case study aims to investigate technological progress of supercomputer development to identify the innovative potential of three leading technology paths toward Exascale development: hybrid system, multicore system and manycore system. 


\subsection{Ex Post Analysis: Revisit EARlier StUdies}

\subsubsection{RiSK ANALYSIS: COMMERCIAL AIRPLANE DEVELOPMENT}

\section{A. RESEARCH FRAMEWORK}

To illustrate the use of the proposed method, this section assumes a scenario in which commercial airplane developers are examining four design concepts in 2007 . They have collected data, including 24 aircrafts introduced to the market in the last 40 years, and attempt to identify which market segment proposed design concepts are subject to and when the ideal delivery to the market would be as competitive products considering the rate of technological advancement observed until 2007.

Note that the performance characteristics used in the earlier study by Lamb, Daim, and Anderson were adopted [225]. In the original study [242], they attempted to develop technology assessment models based on a multiple-regression analysis. However, the resulting model was confined to only two predictors due to the insufficient statistical significance, which resulted in a high unexplained variability. This study revisits and updates the dataset not only to incorporate the latest information but also to investigate the industry dynamics with a consideration of different SOA trends as suggested in the previous study (see Table 14). 
Table 14 Commercial airplane dataset

\begin{tabular}{lcccccc}
\hline \multicolumn{1}{c}{ Airplane } & $\begin{array}{c}\text { EIS } \\
\text { (year) }\end{array}$ & $\begin{array}{c}\text { Travel } \\
\text { range } \\
(1,000 \mathrm{~km})\end{array}$ & $\begin{array}{c}\text { Passenger } \\
\text { capacity } \\
\left(3^{\text {rd }} \text { class }\right)\end{array}$ & $\begin{array}{c}\text { PFE } \\
\text { (passengers*km/L) }\end{array}$ & $\begin{array}{c}\text { Cruising } \\
\text { speed } \\
(\mathrm{km} / \mathrm{h})\end{array}$ & $\begin{array}{c}\text { Maximum } \\
\text { speed } \\
(\mathrm{km} / \mathrm{h})\end{array}$ \\
\hline DC8-55 & 1965 & 9.205 & 132 & 13.721 & 870 & 933 \\
DC8-62 & 1966 & 9.620 & 159 & 16.646 & 870 & 965 \\
$747-100$ & 1969 & 9.800 & 366 & 19.559 & 893 & 945 \\
$747-200$ & 1971 & 12.700 & 366 & 23.339 & 893 & 945 \\
DC10-30 & 1972 & 10.010 & 250 & 18.199 & 870 & 934 \\
DC10-40 & 1973 & 9.265 & 250 & 16.844 & 870 & 934 \\
L1011- 500 & 1979 & 10.200 & 234 & 19.834 & 892 & 955 \\
747-300 & 1983 & 12.400 & 412 & 25.652 & 902 & 945 \\
$767-200 E R$ & 1984 & 12.200 & 181 & 24.327 & 849 & 913 \\
$767-300 E R$ & 1988 & 11.065 & 218 & 26.575 & 849 & 913 \\
$747-400$ & 1989 & 13.450 & 416 & 25.803 & 902 & 977 \\
MD-11 & 1990 & 12.270 & 293 & 24.595 & 870 & 934 \\
A330-300 & 1993 & 10.500 & 295 & 31.877 & 870 & 913 \\
A340-200 & 1993 & 15.000 & 261 & 25.252 & 870 & 913 \\
A340-300 & 1993 & 13.700 & 295 & 27.335 & 870 & 913 \\
MD-11ER & 1996 & 13.408 & 293 & 24.939 & 870 & 934 \\
777-200ER & 1997 & 14.305 & 301 & 25.155 & 892 & 945 \\
777-300 & 1998 & 11.120 & 365 & 23.713 & 892 & 945 \\
A330-200 & 1998 & 12.500 & 253 & 22.735 & 870 & 913 \\
A340-600 & 2002 & 14.600 & 380 & 28.323 & 881 & 913 \\
A340-500 & 2003 & 16.700 & 313 & 24.334 & 881 & 913 \\
777-300ER & 2004 & 14.685 & 365 & 29.568 & 892 & 945 \\
777-200LR & 2006 & 17.370 & 301 & 28.841 & 892 & 945 \\
A380-800 & 2007 & 15.200 & 525 & 24.664 & 902 & 945 \\
\hline EIS: entry into service, PFE: Passenger fuel efficiency derived from passenger capacity, maximum travel range at \\
full payload, and fuel capacity & & & & \\
& & & & & & \\
\hline
\end{tabular}

Table 15 Local RoC of SOA airplanes at the frontier year of 2007

\begin{tabular}{lll}
\hline SOA Airplane & Local RoC & Dominated airplanes \\
\hline $747-300$ & 1.000949 & DC8-55, 747-100/200, L1011-500 \\
$747-400$ & 1.001404 & DC8-55/62, 747-100/200, L1011-500, A340-200 \\
A330-300 & 1.002188 & $767-300 E R$, A340-300 \\
$777-300 E R$ & 1.002561 & $767-300 E R$, A340-200/300/600 \\
$777-200 L R$ & 1.004606 & A340-200/500 \\
A380-800 & 1.003989 & A340-500/600 \\
\hline
\end{tabular}




\section{B. ANALYSIS OF THE CURRENT STATE-OF-THE-ART}

The commercial aircraft industry has important niches with segmented levels of competition from regional jets to jumbo jets. Following the scenario, Table 15 records the local RoCs of six SOA airplanes from the vantage point of 2007. The third column lists dominated airplanes, i.e., past airplanes that have appointed the airplane in the first column as a benchmark. As previously discussed, one can notice that airplanes are grouped together with similarities in their specifications, which characterizes distinct segments in 2007. While the frontier is five dimensional in this application, airplanes in the first column are equivalent to products $B, C, D$, and $E$ from Fig. 10, and column three's airplanes are obsolete airplanes such as $A$.

The Boeing 747 series, as its nickname "jumbo jet" suggests, has been recognized as the most successful wide-body commercial aircraft [243]. In particular, despite their large bodies, the advanced aerodynamic design still allowed the 747-300 and 747-400 to reach a cruising speed of up to $902 \mathrm{~km} / \mathrm{h}$ [244]. These characteristics can be identified from the dominated airplanes that include not only 747 predecessors (747-100 and 747-200) but also Douglas' DC8 series and Lockheed L-1011 that were also known as fast-cruising airplanes. However, gradual technology advancement is observed from the relatively slow local RoC of the 747 aircrafts, which is consistent with the fact that they had been a dominant design for a long time until Airbus created a strong market rival [243].

The Airbus series (A3X0) can be best characterized as long-range airplanes. In fact, the company has primarily targeted the growing demand for high capacity and transcontinental flights. In addition, they have focused their effort at enhancing the structural design using advanced winglets and working on aerodynamic improvements 
for higher fuel efficiency [245]. For example, recent long-range airplanes, the twinjet A330 and the four-engine A340, became popular for their efficient wing design [246]. Meanwhile, the Airbus A340-500 has an operating range of $16,700 \mathrm{~km}$, which is the second longest range of any commercial jet after the Boeing 777-200LR (range of 17,370 $\mathrm{km})$ [247]. Therefore, it is not surprising that the A330-300 has been selected as a benchmark of not only the same family airplane A340-300 but also the Boeing 767300ER, which is also a relatively long-range $(11,065 \mathrm{~km})$ airplane with high passenger fuel efficiency (26.575 passenger*km/L). Additionally, the Airbus A380-800 became the world's largest passenger airplane with a seating capacity of 525 [248]. One can also relate this feature to the reference set which consists of its predecessors: A340-500 and A340-600 with relatively higher passenger capacities as well. This long-range, wide-body airplane has emerged as a fast-growing segment as airlines emphasized transcontinental aircraft capable of directly connecting any two cities in the world [243]. This, in fact, has initiated a series of introductions of the A340 family for Airbus to compete with Boeing [249], which is consistent with the fast local RoCs, indicating a very competitive segment of the market with rapid improvement.

The Boeing 777 series ranks as one of Boeing's best-selling aircraft for their high fuel efficiency, which enable long-range routes [250]. In particular, the 777-300ER is the extended range version of the 777-300, which has a maximum range of $14,685 \mathrm{~km}$, made possible by superior passenger fuel efficiency of 29.568 passenger*km/L. These exceptional characteristics made not only the preceding 767-300ER but also the Airbus series that pursued higher fuel efficiency (A340-200/300/600) appoint the 777-300ER as a benchmark for their performance evaluation. Likewise, the 777-200LR has been 
selected as a benchmark for long-range airplanes that have relatively smaller passenger capacities: A340-200 and A340-500. Because of rising fuel costs, airlines have asked for a fuel-efficient alternative and have increasingly deployed the aircraft on long-haul transoceanic routes [251]. This has driven engineering efforts more toward energy efficient aircraft, which is reflected in the fast local RoCs of the Boeing 777 series.

\section{RISK ANALYSIS}

I now turn to the strategic planning for the proposed airplane concepts (see Table 16). In particular, the planning team would like to identify the relevant engineering targets for each design concept as well as the corresponding rate of technological advancement, i.e., individualized RoC, so that they can examine the feasibility of proposed design concepts in terms of their delivery target.

As SOA airplanes at the frontier of 2007 represent different types of past airplanes, future airplanes, namely design concepts, can be classified by the characteristics of their reference airplanes identified on the 2007 frontier. This allows the model to compute an individualized RoC under which each future airplane is expected to be released. Figure 13 summarizes the results.

Table 16 Four airplane concepts in 2007

\begin{tabular}{ccccccc}
\hline $\begin{array}{c}\text { Design } \\
\text { concept }\end{array}$ & $\begin{array}{c}\text { Travel } \\
\text { range } \\
(1,000 \mathrm{~km})\end{array}$ & $\begin{array}{c}\text { Passenger } \\
\text { capacity } \\
\left(3^{\text {rd }} \text { class }\right)\end{array}$ & $\begin{array}{c}\text { PFE } \\
(\text { passengers*km/L) }\end{array}$ & $\begin{array}{c}\text { Cruising } \\
\text { speed } \\
(\mathrm{km} / \mathrm{h})\end{array}$ & $\begin{array}{c}\text { Maximum } \\
\text { speed } \\
(\mathrm{km} / \mathrm{h})\end{array}$ & $\begin{array}{c}\text { Delivery } \\
\text { target } \\
(\text { year })\end{array}$ \\
\hline 1 & 14.816 & 467 & 28.950 & 917 & 988 & 2010 \\
2 & 15.750 & 280 & 34.851 & 913 & 954 & 2010 \\
3 & 15.000 & 315 & 34.794 & 903 & 945 & 2013 \\
4 & 14.800 & 369 & 35.008 & 903 & 945 & 2015 \\
\hline
\end{tabular}


The first design concept aims for a large commercial aircraft carrying 467 passengers while having the fast cruising speed of $917 \mathrm{~km} / \mathrm{h}$. As noted earlier, these characteristics are also reflected in its reference airplanes: 747-400, 777-300ER, and A380-800. That is, this design concept would compete with these three airplanes in the current (2007) market with given specifications. The individualized RoC of this design concept can therefore be obtained by interpolating local RoCs in conjunction with reference information. Here, the individualized RoC obtained was 1.002748, which suggests a more rapid technology development in its category compared to the average RoC of 1.002149 . This is about $28 \%$ faster and resulted in an estimated entry into service (EIS) of the current design concept in 2011.49. Therefore, one may consider the delivery target of 2010 to be an aggressive goal that might encounter technical challenges by outpacing the rate of technological advancement of the past.

In a similar manner, characteristics of the second design concept's long range of $15,750 \mathrm{~km}$ with outstanding passenger fuel efficiency of 34.851 passenger* $\mathrm{km} / \mathrm{L}$ are consistent with the nature of its identified reference airplanes: A330-300 and 777-200LR. As implied in the local RoCs of 777-200LR (1.004606) with its reference information (0.67), this concept is subject to one of the fastest advancing technology clusters seeking a high fuel efficiency. Consequently, it was expected that the very fast individualized RoC of 1.003793 could achieve this level of specification by 2013.45. Similar to the first design concept, this indicates that the delivery target of 2010 may involve a significant technical risk since it requires exceeding the past rate of technological advancement. 


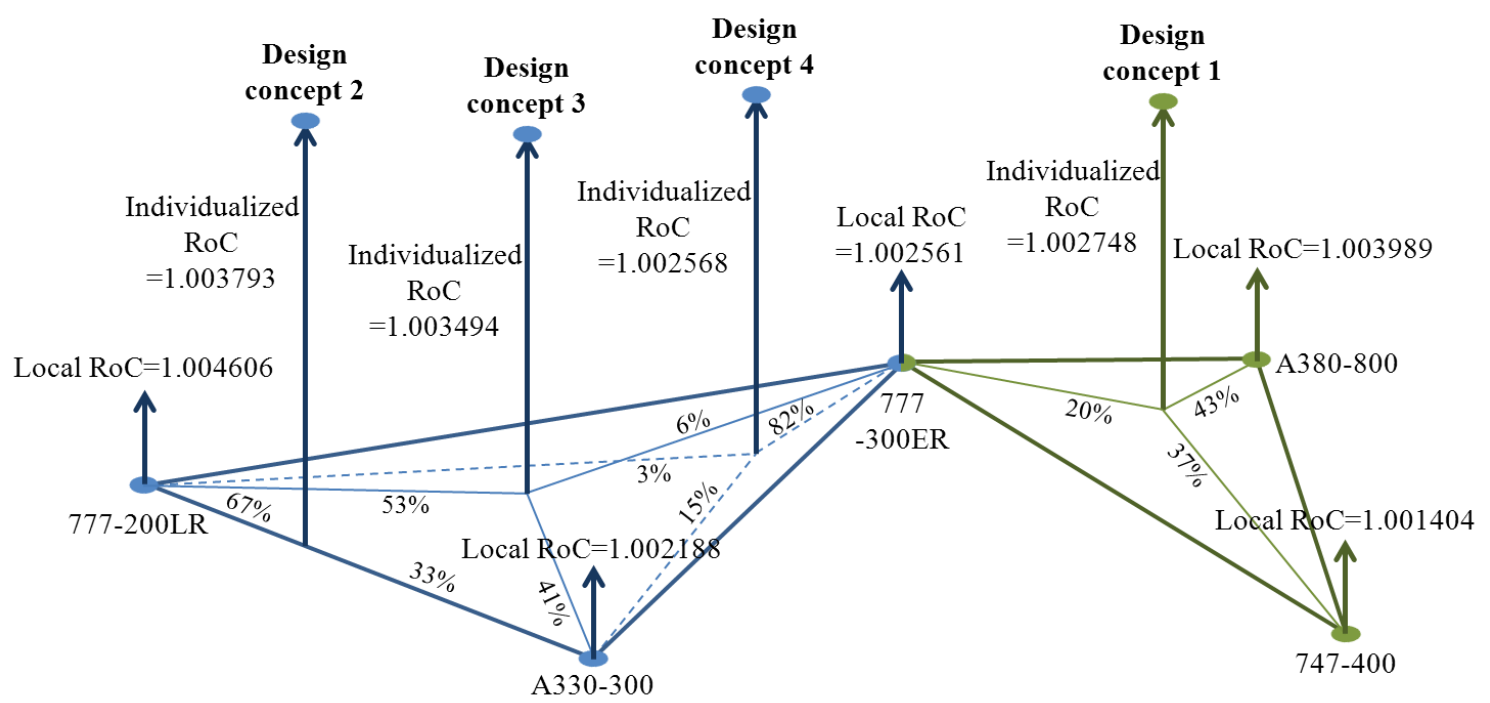

Figure 132007 state-of-the-art frontier with regard to four design concepts ${ }^{6}$

The third design concept has features similar to the second design concept such that it also aims to be a long-range and fuel efficient aircraft; however, it pursues a larger passenger capacity of 315 . This feature is reflected in the reference set that additionally includes 777-300ER, which has a large passenger capacity of 365 . The relatively slow local RoC of the 777-300ER and the A330-300 may imply the difficulty of technological advancement with respect to the travel range and passenger capacity. As a result, the individualized RoC for this design concept was found to be 1.003494, giving a forecasted EIS of 2012.45. Given the delivery target of 2013, the current design concept might be regarded as a feasible goal; however, on the other hand this possibly entails a modest market risk of lagging behind in the performance competition.

The last design concept is a variation of the third design concept, aiming for a much larger airplane but with a shortened travel range. Not surprisingly, this different blend of

\footnotetext{
${ }^{6}$ This figure depicts conceptualized frontier facets relevant to the four design concepts under discussion.
} 
the same three peers makes a virtual target of this design concept positioned closer to the 777-300ER than to the 777-200LR and A330-300, which would result in a further conservative prospect based on the slow rate of performance improvement represented by the 777-300ER. Consequently, the individualized RoC was found to be 1.002568 , giving a forecasted EIS of 2020.16. This indicates that the delivery target of 2015 may be an overly optimistic goal which could cause a postponement due to technical risks involved.

\section{PROOF OF CONCEPT}

I now come back to the present and validate the performance of the presented method (see Table 17). The first design concept was the Boeing 747-8, which began deliveries in 2012 [252]. In fact, this airplane faced two years of delays since its original plan of 2010 due to assembly and design problems followed by contractual issues [253].

The second design concept was another Boeing airplane, 787-9, which made its maiden flight in 2013, and the delivery began on July 2014 [254], [255]. In line with the results, the originally targeted EIS of 2010 could not be met because of multiple delays due to technical problems in addition to a machinists' strike [256].

The third design concept was the initial design target of Airbus A350-900, which has been changed and rescheduled to enter service in the second half of 2014 [257], [258]. The delay was mainly imposed by a strategic redesign of the A350, the so-called XWB (extra-wide-body) program, that allows for a maximum seating capacity of 440 with a 10abreast high-density seating configuration as well as a reinforced fuselage design [259]. It is interesting to note that Airbus has made a strategic decision by delaying the A350- 
900's delivery with improved specifications to compete with the Boeing 777 series in the jumbo jet segment, which was recognized in the analysis results seven years ago.

Similarly, the last design concept was the Airbus A350-1000, whose EIS has also been rescheduled to 2018 [260]. This airplane is the largest variation of the A350 family and designed to compete with the Boeing 777-300ER, as is also seen from the reference information. Nevertheless, the postponed delivery target of 2018 may still be an aggressive goal considering the technological advancement observed in this segment.

Table 17 Results summary

\begin{tabular}{llccc}
\hline $\begin{array}{l}\text { Design } \\
\text { concept }\end{array}$ & Reference airplanes (competitors) & $\begin{array}{c}\text { Planned } \\
\text { EIS }\end{array}$ & $\begin{array}{c}\text { Estimated } \\
\text { EIS }\end{array}$ & $\begin{array}{c}\text { Delayed } \\
\text { EIS }\end{array}$ \\
\hline $1(747-8)$ & 747-400, 777-300ER, A380-800 & 2010 & 2011.49 & 2012 \\
$2(787-9)$ & A330-300, 777-200LR & 2010 & 2013.45 & 2014 \\
$3($ A350-900*) & A330-300, 777-300ER, 777-200LR & 2013 & 2012.45 & 2014 \\
$4($ A350-1000) & A330-300, 777-300ER, 777-200LR & 2015 & 2020.16 & 2018 \\
\hline & & & &
\end{tabular}




\subsubsection{VALIDATION USING PAST DATASETS}

\section{A. Forecasting ACCURACY EVALUATION TECHNIQUES}

Fundamentally, the true accuracy of a forecasting model should be judged by the future events that were not known during the model building process. However, this socalled "real time assessment" has practical limitations for practitioners, which makes a holdout sample test that measures how the model is able to reproduce data already known but not used in construction of the model commonplace in forecasting literature [261]. The resulting forecast deviations, i.e., the difference between estimated data and the held out data, can therefore provide an accuracy measure (or the goodness of fit) of the forecast model being considered. This is also useful to compare the performance of different models on the same data [262].

A holdout sample test requires the division of the historical data series into a fit period and a test period. The forecasting origin is defined as the point from which the forecasts are generated. In general, the practitioner can adopt either a single forecasting origin or multiple forecasting origins.

Forecast statistics relying on a single forecasting origin, i.e., a fixed origin evaluation, often require a fairly long test period since they yield only one set of forecasts from a given forecasting origin. This necessarily renders the resulting summary statistics to be a mélange of near-term and far-term forecast errors [263]. In addition, practitioners must justify the reason to pick only one set of forecasts, which otherwise could raise a criticism such that the results are susceptible to corruption by occurrences unique to that origin [261]. 
To overcome the aforementioned problems of using a single origin, a rolling origin evaluation successively updates the forecasting origin and accumulates forecasts from each new origin. This technique makes it possible not only to obtain a sufficient number of forecasts with the same historical data but also to desensitize the error measures to special events at any single origin [264]. Furthermore, a rolling origin evaluation produces multiple forecasts for every lead time, i.e., time period, between the origin and the time being forecasted, which allows one to assess the forecasting accuracy of an individual times series at each lead time [265].

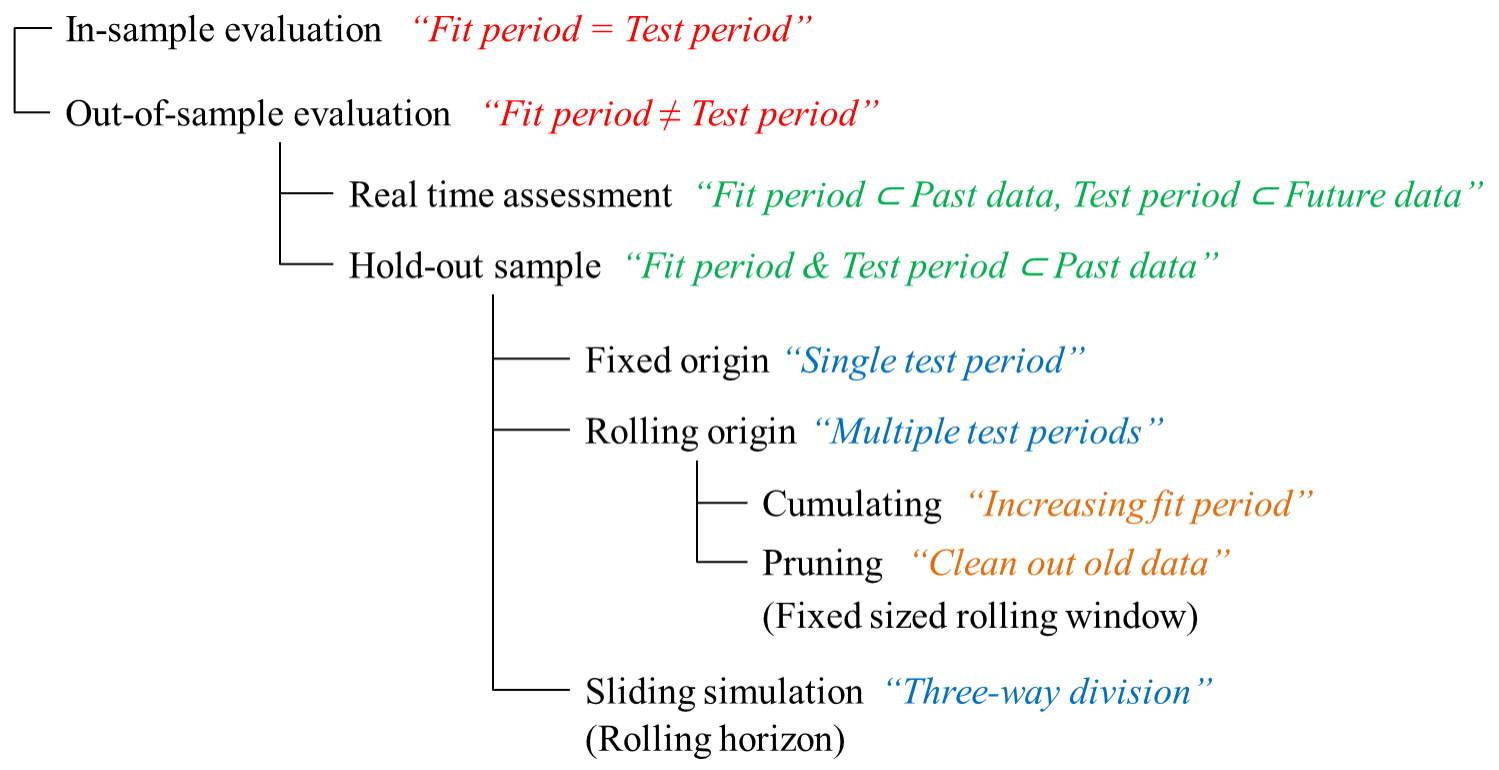

Figure 14 Various forecast accuracy tests

Swanson and White's study discussed that forecasting accuracy may also be affected by an increase of the fit period when a rolling origin is employed [266]. To avoid this, they suggested a procedure called a fixed size rolling window to maintain a constant 
length of the fit period. This technique can clean out old data in an attempt to update the forecasting model, thereby mitigating the influence of data from the distant past [261].

\section{B. TEST RESULTS FROM EARLIER STUDIES ${ }^{7}$}

To validate the performance of the proposed approach, holdout sample tests using both constant RoC and segmented RoC on six datasets were conducted. Note that a rolling origin evaluation was implemented from the origin used in the original studies. It should be also noted here that the accuracy measure of root mean square error (RMSE) was adopted to represent forecasting errors since our forecast is the arrival of technologies, i.e., single scale with non-zero occurrence, estimated from their performance levels [267]. In addition, deviation distributions were tested to distinguish their differences from random variations with statistical significance. Table 18 summarizes comparative results of forecasting accuracies.

Table 18 Forecast accuracy comparisons

\begin{tabular}{l|c|c|c|c|c|c}
\hline \multirow{2}{*}{\multicolumn{1}{c|}{ Application area }} & \multicolumn{2}{c|}{$\begin{array}{c}\text { RMSE } \\
\text { (Root mean square error) }\end{array}$} & $\begin{array}{c}\text { Deviation statistics } \\
\text { 95\% confidence interval) }\end{array}$ & \multicolumn{2}{c}{ Paired $t$ test } \\
\cline { 2 - 6 } & $\begin{array}{c}\text { Constant } \\
\text { RoC }\end{array}$ & $\begin{array}{c}\text { Segmented } \\
\text { RoC }\end{array}$ & $\begin{array}{c}\text { Constant } \\
\text { RoC }\end{array}$ & $\begin{array}{c}\text { Segmented } \\
\text { RoC }\end{array}$ & $t$-stat & $p$-value \\
\hline Commercial airplane [225] & 11.9208 & 6.3084 & $-9.06( \pm 5.18)$ & $-3.56( \pm 3.65)$ & -4.3653 & 0.0023 \\
Fighter jet [230] & 7.8229 & 7.2524 & $-7.22( \pm 3.38)$ & $-6.32( \pm 3.17)$ & -2.1274 & 0.0454 \\
Battle tank [224] & 23.1312 & 16.7987 & $-15.57( \pm 7.62)$ & $-9.30( \pm 6.30)$ & -5.3973 & 0.0001 \\
LCD [232] & 2.3061 & 2.1508 & $+0.63( \pm 0.27)$ & $+0.35( \pm 0.30)$ & 6.7182 & 0.0000 \\
HEV [268] & 3.4176 & 3.3329 & $-2.33( \pm 1.70)$ & $-2.26( \pm 1.67)$ & -3.2221 & 0.0105 \\
DSLR [269] & 2.6333 & 2.6271 & $-0.43( \pm 0.36)$ & $-0.15( \pm 0.33)$ & -3.8553 & 0.0002 \\
\hline
\end{tabular}

${ }^{7}$ This section is adapted from a paper accepted in proceedings of PICMET'14 [317] 
In all cases, the segmented $\mathrm{RoC}$ showed not only smaller forecasting errors, i.e., $R M S E_{\text {Constant RoC }}>R M S E_{\text {Segmented RoC }}$, but also statistically significant distributions closer to zero than that of constant RoC, i.e., $\left|\mu_{\text {Constant RoC }}^{\text {Deviation }}\right|>\left|\mu_{\text {Segmented RoC }}^{\text {Deviation }}\right|(p<0.05)$. One may infer that forecasting accuracy improvement would be more significant if unique segments were identified with a greater local RoC contrast to one another, and future technologies were subject to those unique segments. This can be shown by comparing the constant RoC with individualized RoCs.

Figure 15 contains this information. Note that RoCs were normalized to show their distribution in comparison to constant RoCs that were set to be $100 \%$ across the forecasting origins. It is seen that in the case of commercial airplane and battle tank applications, individualized RoCs for forecasting targets show skewed distributions from constant RoCs. That is, most of the forecasting targets were subject to relatively fast progressing segments such that constant RoCs had to yield fairly conservative forecasts as seen from the deviation statistics. On the other hand, the segmented RoC approach could reflect those variations by obtaining fast individualized RoCs from the distribution of local RoCs, which resulted in considerable accuracy improvements.

In contrast, when the local RoC of a certain segment by which most future technologies are classified was close to the constant RoC, the impact of segmented RoC would be marginal even if a wide range of local RoCs was identified. This can be seen from the case of DSLR application in which a constant RoC could reasonably represent the variations of individualized RoCs as an average value. 

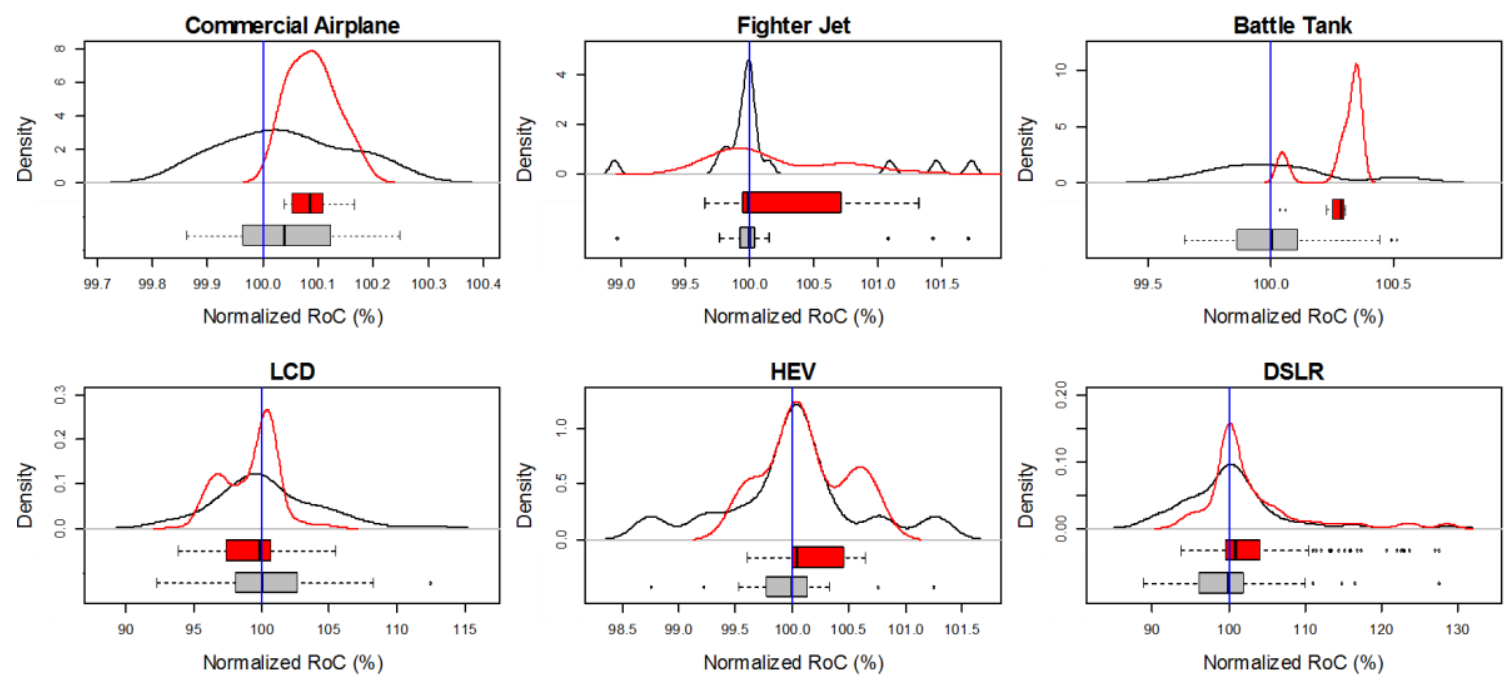

- Local RoC

— Individualized RoC

- Constant (average) RoC

Figure 15 Relative comparison of segmented RoC with constant RoC

A special case can occur when the regions or clusters do not contain past products that have been surpassed. In this case, a product may not have a local RoC. Graphically, this would occur in Fig. 9 if products B and E were not included, which would then result in $\mathrm{G}$ failing to have a local RoC. In such cases, G's local RoC could be assumed to be the average RoC of all SOA products ( $\mathrm{H}$ and $\mathrm{I})$. Another approach would be to average the RoC for products that are on the same facet(s) of the efficiency frontier (simply $\mathrm{H}$ ). 


\subsection{Ex Ante Analysis: Focused Applications}

In the previous section, the proposed model was tested based on historical data. In contrast, this section provides a focused case study of supercomputer development to demonstrate how the model can be used in a predictive manner.

\subsubsection{EXASCALE SUPERCOMPUTER DEVELOPMENT ${ }^{8}$}

\section{A. BACKGROUND}

Supercomputers have played a critical role in various fields which require computationally intensive tasks such as pharmaceutical testing, genomics research, climate simulation, energy exploration, molecular modeling, astrophysical simulation, etc. The unquenchable need for faster and higher precision analysis in those fields creates the demand for even more powerful supercomputers. Furthermore, developing an indigenous supercomputer industry has become a fierce international competition due to its role as a strategic asset for a nationwide scientific research and the prestige of being the maker of the fastest computers [270], [271]. While the vast majority of supercomputers have still been built using processors from Intel, Advanced Micro Devices (AMD), and Nvidia, manufacturers are committed to developing their own customized systems, e.g., interconnect, operating system and resource management, as system optimization becomes a crucial factor in today's massively parallel computing paradigm [272].

Advances in supercomputers have come at a steady pace over the past 20 years in terms of speed, which has been enabled by the continual improvement in computer chip manufacturing [273]. As of March 2014, the world's fastest supercomputer is the Tianhe-

\footnotetext{
${ }^{8}$ This section is adapted from a paper accepted in Omega [318]
} 
2 built by China's National University of Defense Technology (NUDT) performing at over 33.86 Petaflops, i.e., $33.86 \times 10^{15}$ floating point operations per second. This is about 273,000 times faster than the fastest machine from 20 years ago, the Fujitsu Numerical Wind Tunnel. On average, progress went from being measured by Gigaflops in 1990 to Teraflops in about 10 years, and then to Petaflops in another 10 years [274]. In line with this, the next milestone is to build an Exascale computer, a machine capable of doing a quintillion operations, i.e., $10^{18}$, per second, which had been projected to see light of day by 2018 [275]. However, there are significant industry concerns that this incremental improvement might not continue mainly due to several practical problems.

The biggest challenge to build the Exascale computer is the power consumption [276]. Tianhe-2, which is currently not only the fastest but also the most power hungry supercomputer, uses about 18 megawatts (MW) of power. If the current trend of power use continues, projections for the Exascale computing systems range from 60 to $130 \mathrm{MW}$, which would cost up to $\$ 150$ million annually [277]. Furthermore, few sites in the U.S. will be able to host such power hungry computing systems due to the limited availability of facilities with sufficient power and cooling capabilities [278]. Therefore, unlike past advancement mainly driven by performance improvement [279], power efficiency has now gone from being a negligible factor to a fundamental design consideration. To cope with these issues, current efforts are targeting the Exascale machine that uses electrical power of $20 \mathrm{MW}$ using 100 million processors in the 2020 timeframe [276], [280].

Figure 16 illustrates this challenge. The rate of performance progress has been constant until a recent date, and this would envision the first Exascale computer in 2018. 
However, this steady performance improvement has been made possible by meeting the exponentially growing power demands. Now that the $20 \mathrm{MW}$ of power consumption is set as a feasible limit, the engineering effort has to be focusing more on minimizing power consumption than on maximizing computational power. This implies that extrapolation relying on a single performance measure, i.e., computing speed, may overlook required features of future technology systems and could eventually result in an erroneous forecast. Specifically, the average power efficiency of today's top 10 systems is about 2.2 Petaflops per megawatt. This indicates that it is required to improve power efficiency by a factor of 23 to achieve the Exascale goal. It is therefore crucial to incorporate the power consumption and multicore characteristics that identify the power efficiency into the measure of technology assessment to have a comprehensive understanding of future high performance computing (HPC) [281]. This requires a multifaceted approach to investigate the tradeoffs between system attributes, which can tackle questions such as: How much performance improvement would be restricted by power and/or core reduction? What would be the maximum attainable computing performance with certain levels of power consumption and/or the number of cores?
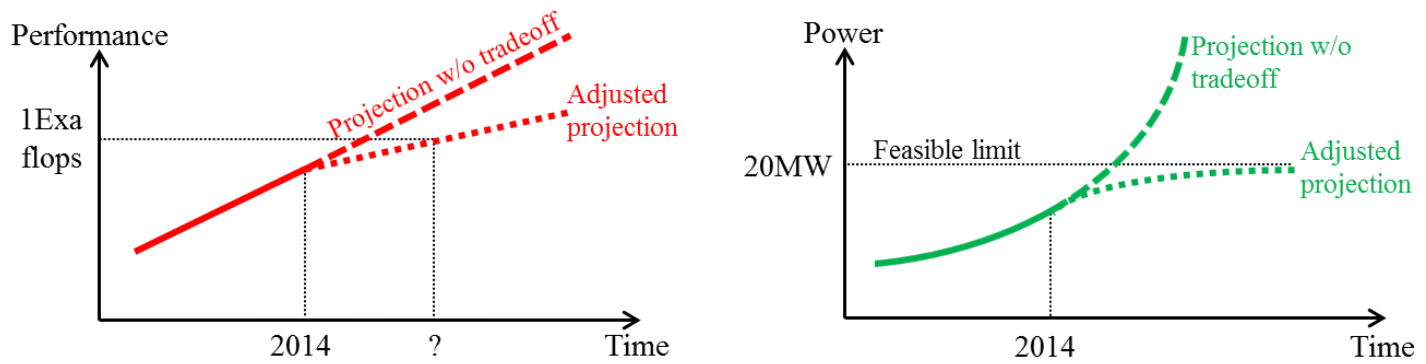

Figure 16 System tradeoffs to be considered for the future HPC trend 
There are three leading technology paths representing today's supercomputer development: hybrid systems, multicore systems, and manycore systems [282]. The hybrid systems use both central processing units (CPU) and graphics processing units (GPU) to efficiently leverage the performances [283]. The multicore systems maintain a number of complex cores, whereas the manycore systems use a large number of less powerful but power efficient cores within the highly parallel architecture [284]. Manufacturers and researchers are exploring these alternate paths to identify the most promising, namely energy efficient and performance effective, avenue to face challenges of deploying and managing Exascale systems [285]-[287]. The comparative analysis of these technology paths can, therefore, give insights into the estimation of the future performance levels as well as the possible disruptive technology changes.

This study employs Technology Forecasting using Data Envelopment Analysis (TFDEA) to measure the technological progress considering tradeoffs among power consumption, multicore processors, and maximum performance so that supercomputers are to be evaluated in terms of both power efficiency and performance effectiveness. The resulting analysis then provides a forecast of Exascale computer deployment under three different development alternatives in consideration of the current business environment as well as emerging technologies. 


\section{B. ANALYSIS}

\section{a) Dataset}

The TOP500 list was first created in 1993 to assemble and maintain a list of the 500 most powerful computer systems [288]. Since the list has been compiled twice a year, datasets from 1993 to 2013 have been combined and cleaned up so that each machine appears once in the final dataset. The purpose of this study is to consider both power efficiency and performance effectiveness; therefore, lists up to 2007 were excluded due to the lack of information on the power consumption (see table 19). Variables selected for this study are as follows:

- Name (text): name of machine

- Year (year): year of installation/last major update

- Total cores (number): number of processors

- Rmax (Gigaflops): maximal LINPACK performance achieved

- Power (Kilowatts): power consumption

- Processor technology/family (text): processor architecture being used

- Interconnect family (text): interconnect being used

In the final dataset, there were a total of 1,199 machines, with the number of cores ranging from 960 to 3.12 million, power ranging from $19 \mathrm{KW}$ to $17.81 \mathrm{MW}$, and Rmax ranging from 9 Teraflops to 33.86 Petaflops from 2002 to 2013. Note that a logarithmic transformation was applied to all three variables due to their exponentially increasing trends. 
Table 19 TOP500 dataset from 1993 to 2013

\begin{tabular}{|c|c|c|c|c|c|c|}
\hline Data column & $\begin{array}{c}1993 \\
\sim 2007\end{array}$ & $\begin{array}{c}2008 \\
\sim 2009\end{array}$ & $2010-1^{*}$ & $2010-2^{*}$ & $2011-1^{*}$ & $\begin{array}{c}2011-2^{*} \\
\sim 2013\end{array}$ \\
\hline Rank & $\mathrm{O}$ & $\mathrm{O}$ & $\mathrm{O}$ & $\mathrm{O}$ & $\mathrm{O}$ & $\mathrm{O}$ \\
\hline Site & $\mathrm{O}$ & $\mathrm{O}$ & $\mathrm{O}$ & $\mathrm{O}$ & $\mathrm{O}$ & $\mathrm{O}$ \\
\hline Manufacturer & $\mathrm{O}$ & $\mathrm{O}$ & $\mathrm{O}$ & $\mathrm{O}$ & $\mathrm{O}$ & $\mathrm{O}$ \\
\hline Name & $x$ & $x$ & $x$ & $x$ & $x$ & $\mathrm{O}$ \\
\hline Computer & $\mathrm{O}$ & $\mathrm{O}$ & $\mathrm{O}$ & $\mathrm{O}$ & $\mathrm{O}$ & $\mathrm{O}$ \\
\hline Country & $\mathrm{O}$ & $\mathrm{O}$ & $\mathrm{O}$ & $\mathrm{O}$ & $\mathrm{O}$ & $\mathrm{O}$ \\
\hline Year & $\mathrm{O}$ & $\mathrm{O}$ & $\mathrm{O}$ & $\mathrm{O}$ & $\mathrm{O}$ & $\mathrm{O}$ \\
\hline Total Cores & $\mathrm{O}$ & $\mathrm{O}$ & $\mathrm{O}$ & $\mathrm{O}$ & $\mathrm{O}$ & $\mathrm{O}$ \\
\hline Accelerator/Co-Processor Cores & $x$ & $x$ & $x$ & $x$ & $x$ & $\mathrm{O}$ \\
\hline $\operatorname{Rmax}$ & $\mathrm{O}$ & $\mathrm{O}$ & $\mathrm{O}$ & $\mathrm{O}$ & $\mathrm{O}$ & $\mathrm{O}$ \\
\hline Rpeak & $\mathrm{O}$ & $\mathrm{O}$ & $\mathrm{O}$ & $\mathrm{O}$ & $\mathrm{O}$ & $\mathrm{O}$ \\
\hline Efficiency (\%) & $x$ & $x$ & $x$ & $x$ & $x$ & $\mathrm{O}$ \\
\hline Nmax & $\mathrm{O}$ & $\mathrm{O}$ & $\mathrm{O}$ & $\mathrm{O}$ & $\mathrm{O}$ & $\mathrm{O}$ \\
\hline Nhalf & $\mathrm{O}$ & $\mathrm{O}$ & $\mathrm{O}$ & $\mathrm{O}$ & $\mathrm{O}$ & $\mathrm{O}$ \\
\hline Power & $x$ & $\mathrm{O}$ & $\mathrm{O}$ & $\mathrm{O}$ & $\mathrm{O}$ & $\mathrm{O}$ \\
\hline Mflops/Watt & $x$ & $x$ & $x$ & $x$ & $x$ & $\mathrm{O}$ \\
\hline Measured Size & $x$ & $x$ & $\mathrm{O}$ & $x$ & $x$ & $x$ \\
\hline Processor Technology/Family & $\mathrm{O}$ & $\mathrm{O}$ & $\mathrm{O}$ & $\mathrm{O}$ & $\mathrm{O}$ & $\mathrm{O}$ \\
\hline Processor Generation & $x$ & $x$ & $x$ & $x$ & $x$ & $\mathrm{O}$ \\
\hline Processor & $\mathrm{O}$ & $\mathrm{O}$ & $\mathrm{O}$ & $\mathrm{O}$ & $\mathrm{O}$ & $\mathrm{O}$ \\
\hline Proc. Frequency & $\mathrm{O}$ & $\mathrm{O}$ & $\mathrm{O}$ & $\mathrm{O}$ & $\mathrm{O}$ & $x$ \\
\hline Processor Cores & $x$ & $x$ & $\mathrm{O}$ & $\mathrm{O}$ & $\mathrm{O}$ & $x$ \\
\hline Processor Speed (MHz) & $x$ & $x$ & $x$ & $x$ & $x$ & $\mathrm{O}$ \\
\hline System Family & $\mathrm{O}$ & $\mathrm{O}$ & $\mathrm{O}$ & $\mathrm{O}$ & $\mathrm{O}$ & $\mathrm{O}$ \\
\hline System Model & $x$ & $\mathrm{O}$ & $\mathrm{O}$ & $\mathrm{O}$ & $\mathrm{O}$ & $\mathrm{O}$ \\
\hline Operating System & $\mathrm{O}$ & $\mathrm{O}$ & $\mathrm{O}$ & $\mathrm{O}$ & $\mathrm{O}$ & $\mathrm{O}$ \\
\hline OS Family & $x$ & $x$ & $x$ & $x$ & $x$ & $\mathrm{O}$ \\
\hline Cores per Socket & $x$ & $x$ & $x$ & $x$ & $x$ & $\mathrm{O}$ \\
\hline Architecture & $\mathrm{O}$ & $\mathrm{O}$ & $\mathrm{O}$ & $\mathrm{O}$ & $\mathrm{O}$ & $\mathrm{O}$ \\
\hline Accelerator/Co-Processor & $x$ & $x$ & $x$ & $x$ & $\mathrm{O}$ & $\mathrm{O}$ \\
\hline Segment & $\mathrm{O}$ & $\mathrm{O}$ & $\mathrm{O}$ & $\mathrm{O}$ & $\mathrm{O}$ & $\mathrm{O}$ \\
\hline Application Area & $\mathrm{O}$ & $\mathrm{O}$ & $\mathrm{O}$ & $\mathrm{O}$ & $\mathrm{O}$ & $x$ \\
\hline Interconnect Family & $\mathrm{O}$ & $\mathrm{O}$ & $\mathrm{O}$ & $\mathrm{O}$ & $\mathrm{O}$ & $\mathrm{O}$ \\
\hline Interconnect & $\mathrm{O}$ & $\mathrm{O}$ & $\mathrm{O}$ & $\mathrm{O}$ & $\mathrm{O}$ & $\mathrm{O}$ \\
\hline Region & $\mathrm{O}$ & $\mathrm{O}$ & $\mathrm{O}$ & $\mathrm{O}$ & $\mathrm{O}$ & $\mathrm{O}$ \\
\hline Continent & $\mathrm{O}$ & $\mathrm{O}$ & $\mathrm{O}$ & $\mathrm{O}$ & $\mathrm{O}$ & $\mathrm{O}$ \\
\hline
\end{tabular}

O: Available, $x$ : Unavailable * ‘-1(2)' denotes the first (second) list of corresponding years 


\section{b) Model building}

As discussed earlier, power consumption and the number of cores are key variables representing the power efficiency and therefore were used as input variables, while the maximum LINPACK performance (Rmax) was used as the output variable. This allows the model to identify "the better performing" supercomputer which has lower power, fewer cores, and/or higher performance if other factors are held constant.

Orientation can be either input-oriented or output-oriented and can be best thought of as whether the technological progress is better characterized as "input reduction" or "output augmentation" [232]. While power consumption will be a key concern in the Exascale computing, the advancement of this industry has been driven primarily by computing performance, i.e., flops, improvement. In fact, the Exascale computing is a clearly defined development goal, and therefore an output orientation was selected for this application. It should be noted here that either orientation can deal with tradeoffs among input and output variables.

As with many DEA applications, variable returns to scale (VRS) was selected for appropriate returns to scale assumption since doubling the input(s) doesn't correspond to doubling the output(s) here.

The main purpose of this study is to make a forecast of the Exascale computer deployment by examining past rates of progress, thus the frontier year of 2013 was used so as to cover the entire dataset. Lastly, minimizing the sum of effective dates was added as a secondary goal into the model to handle the potential issue of multiple optima from the dynamic frontier year [240]. Table 20 summarizes the model parameters used in this study. 
Table 20 TFDEA model parameters

\begin{tabular}{ccccccc}
\hline Inputs & Output & Orientation & RTS & Frontier year & Frontier type & Second goal \\
\hline Power, Cores & Rmax & Output & VRS & 2013 & Dynamic & Min \\
\hline
\end{tabular}

Figure 17 shows 13 supercomputers identified as SOAs from the analysis. Intel provided the processors for the largest share (62\%) and, inter alia, GPU/Accelerator based systems showed impressive performances both in power and core efficiencies, while IBM's Blue Gene, NNSA/SC and Blue Gene/Q showed comparable power efficiency as manycore based systems.

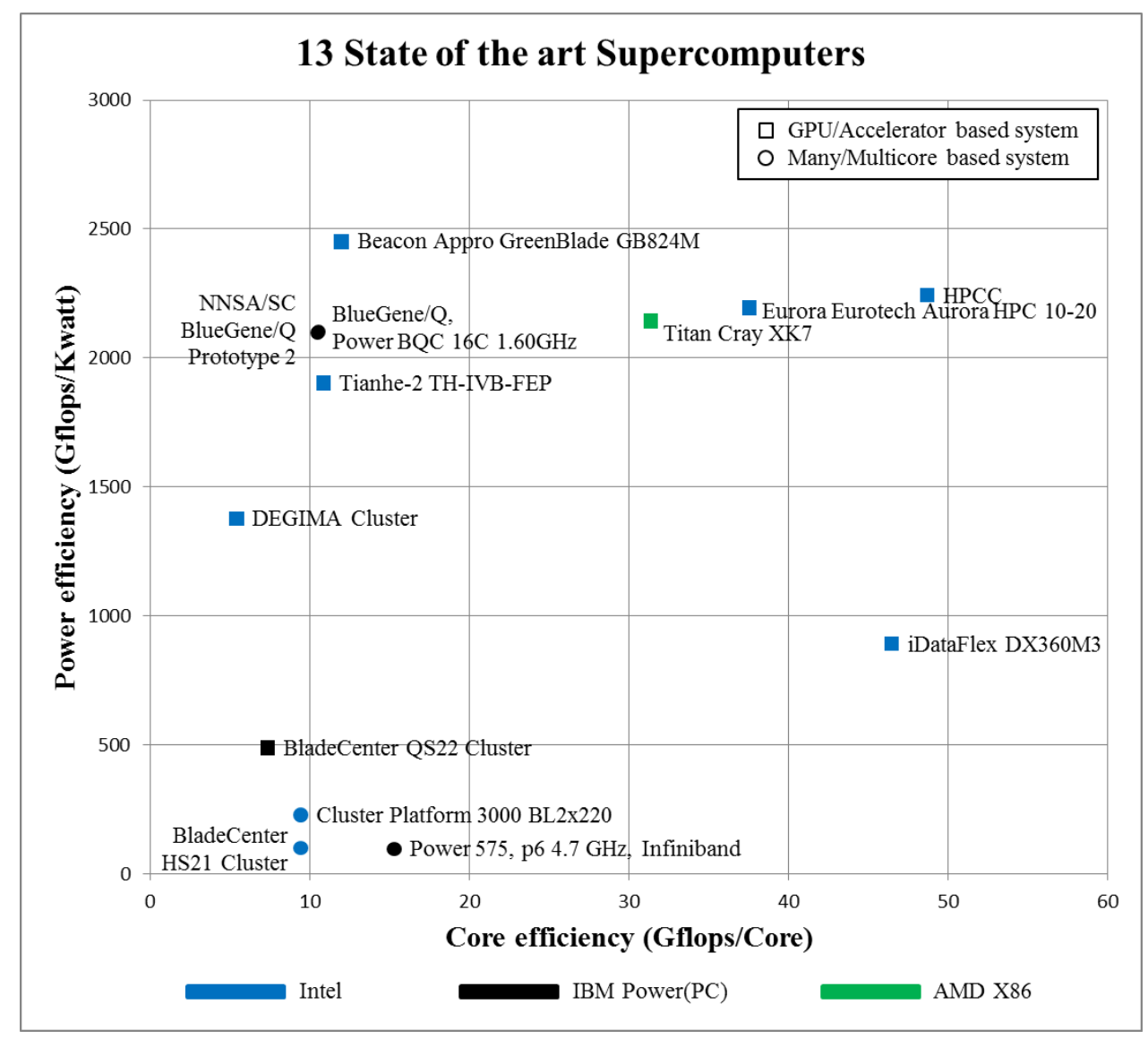

Figure 1713 State-of-the-art supercomputers considering system tradeoffs 
As also seen from specifications of these supercomputers in Table 21, supercomputers in different sizes are characterized as being equally competitive in consideration of system tradeoffs. This enables the model to construct technology frontiers from which various production possibilities can be identified. This characteristic, in fact, differentiates the TFDEA process from a single dimensional measure such as the TOP500 list in which technological efforts to become energy efficient and/or core efficient are not taken into account.

Table 21 Specifications of 13 SOA supercomputers

\begin{tabular}{|c|c|c|c|c|c|c|}
\hline \multirow{2}{*}{ Name } & \multirow{2}{*}{ Year } & \multirow{2}{*}{ Cores } & \multirow{2}{*}{ Power } & \multirow{2}{*}{$R \max$} & \multicolumn{2}{|c|}{ Technology Family } \\
\hline & & & & & Processor & Interconnect \\
\hline $\begin{array}{l}\text { Eurora Eurotech } \\
\text { Aurora HPC 10-20 }\end{array}$ & 2013 & 2,688 & 46.00 & 100,900 & Intel & InfiniBand \\
\hline $\begin{array}{l}\text { Tianhe-2 TH-IVB- } \\
\text { FEP }\end{array}$ & 2013 & $3,120,000$ & $17,808.00$ & $33,862,700$ & Intel & Custom \\
\hline HPCC & 2013 & 10,920 & 237.00 & 531,600 & Intel & InfiniBand \\
\hline Titan Cray XK7 & 2012 & 560,640 & $8,209.00$ & $17,590,000$ & AMD & Cray \\
\hline $\begin{array}{l}\text { Beacon Appro } \\
\text { GreenBlade } \\
\text { GB824M }\end{array}$ & 2012 & 9,216 & 45.11 & 110,500 & Intel & InfiniBand \\
\hline $\begin{array}{l}\text { BlueGene/Q, Power } \\
\text { BQC 16C } 1.60 \mathrm{GHz}\end{array}$ & 2012 & 8,192 & 41.09 & 86,346 & IBM Power & Custom \\
\hline iDataPlex DX360M3 & 2011 & 3,072 & 160.00 & 142,700 & Intel & InfiniBand \\
\hline $\begin{array}{l}\text { NNSA/SC Blue } \\
\text { Gene/Q Prototype } 2\end{array}$ & 2011 & 8,192 & 40.95 & 85,880 & IBM Power & Custom \\
\hline DEGIMA Cluster & 2011 & 7,920 & 31.13 & 42,830 & Intel & InfiniBand \\
\hline $\begin{array}{l}\text { BladeCenter QS22 } \\
\text { Cluster }\end{array}$ & 2008 & 1,260 & 18.97 & 9,259 & IBM Power & InfiniBand \\
\hline $\begin{array}{l}\text { Cluster Platform } \\
3000 \text { BL2x220 }\end{array}$ & 2008 & 1,024 & 42.60 & 9,669 & Intel & InfiniBand \\
\hline $\begin{array}{l}\text { Power 575, p6 } 4.7 \\
\text { GHz, Infiniband }\end{array}$ & 2008 & 960 & 153.43 & 14,669 & IBM Power & InfiniBand \\
\hline $\begin{array}{l}\text { BladeCenter HS21 } \\
\text { Cluster }\end{array}$ & 2007 & 960 & 91.55 & 9,058 & Intel & InfiniBand \\
\hline
\end{tabular}


Figure 18 illustrates performance trajectories based on 1,199 supercomputers from three dominant processor families: AMD, IBM Power, and Intel (IA-32/64, Core, Nehalem, Westmere, and Sandy Bridge). Since the Japanese supercomputer, EarthSimulator, was built in 2002 using a Nippon Electric Company (NEC) chip which was not adopted by other supercomputer manufacturers thereafter, Fig. 18 is drawn from 2005 to focus on the main vendors of processors for today's systems. The ordinate is the overall performance score from the DEA model. As such, each line indicates a performance trajectory of the top performing supercomputers from each year against the frontier year of 2013. A performance score of $100 \%$ indicates that the supercomputer has a superior performance enough to be on the SOA frontier in 2013. A performance score higher than $100 \%$ denotes super-efficiency from the DEA model, which can show how much the supercomputer is outperforming other SOA supercomputers.

The trajectory of Many/Multicore systems shows that IBM Power (PC) processor based machines are outperforming AMD processor based machines. AMD processor based machines, however, showed surpassing performances over IBM Power (PC) based machines when they were adopted by Cray to build hybrid systems in 2011 and 2012. In fact, the successful development of Titan Cray XK7 using AMD Opteron CPUs coupled with Nvidia coprocessors has made Cray Inc. one of the leading supercomputer vendors to date. Interestingly, this is also consistent with the fact that Cray Inc. was awarded the $\$ 188$ M U.S. Blue Waters contract, which is a project funded by the National Science Foundation (NSF), replacing IBM, which had pulled out of the project prior to completion in 2011 [289]. 


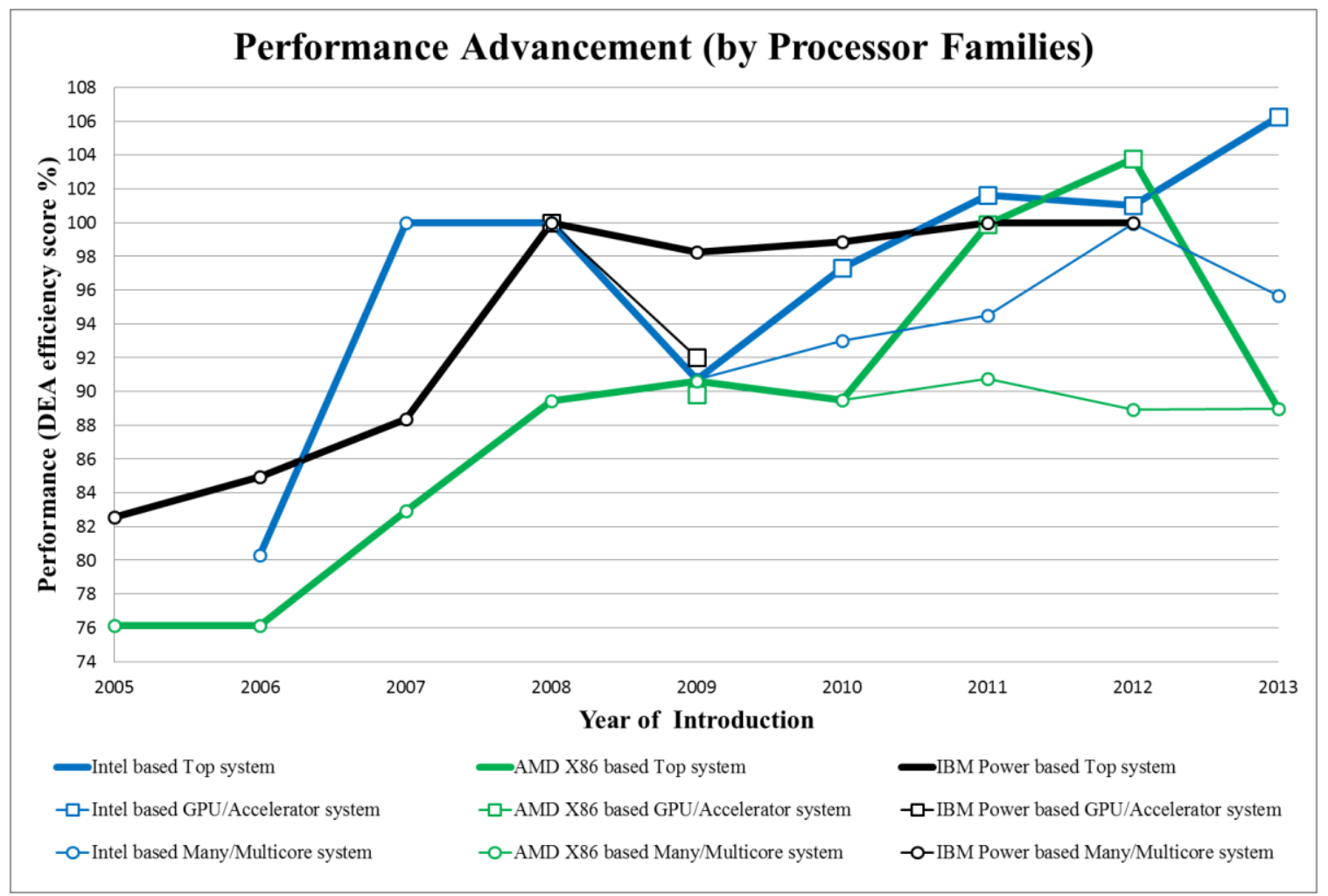

Figure 18 Performance trajectories of different processor families

It is also interesting to point out that there was a distinct performance gap between Many/Multicore based machines and GPU/Accelerator based machines using AMD processors in 2011 and 2012. This can be attributed to the strategic partnership between Cray and AMD. In fact, Cray has been a staunch supporter of AMD processors since 2007, and their collaboration has delivered continued advancement in HPC [290]. In particular, Cray's recent interconnect technology, Gemini, was customized for the AMD Opteron CPUs Hyper-Transport links to optimize internal bandwidth [291]. Since modern supercomputers are deployed as massively centralized parallel systems, the speed and flexibility of interconnect become important for the overall performance of a supercomputer. Given that hybrid machines using AMD processors all use Cray's 
interconnect system, one may notice that AMD based supercomputers had a significant performance contribution from Cray interconnect as well as Nvidia coprocessors.

One may notice that top supercomputers based on Intel processors have switched to hybrid systems since 2010. This is because combining CPUs and GPUs is advantageous in data parallelism, which makes it possible to balance the workload distribution as efficient use of computing resources becomes more important in today's HPC structure [292]. Hybrid machines using Intel processors have all adopted InfiniBand interconnect for their cluster architectures regardless of GPUs/Accelerators: Nvidia, ATI Radeon, Xeon Phi, PowerXCell, etc. InfiniBand, manufactured by Mellanox and Intel, enables low processing overhead and is designed to carry multiple traffic types such as clustering, communications, and storage over a single connection [293]. In particular, its GPUDirect technology facilitates faster communication and lower latency of GPU/Accelerator based systems that can increase computing and accelerator resources, as well as improves productivity and scalable performance [294]. Intel acquired the InfiniBand business from Qlogic in 2012 to support innovating on fabric architectures not only for the HPC but also data centers, cloud, and Web 2.0 market [295].

As another possibility, recent attention is focusing on Intel's next generation supercomputer, which will adopt Cray's Aries interconnect with Intel Xeon Phi accelerator as Intel's first non-InfiniBand based hybrid system after its acquisition of interconnect business from Cray [296]. Interestingly, this transition reflects the strategic decision of Cray, ending an association with AMD to facilitate an independent interconnect architecture rather than a processor specific one as AMD's recent performance and supply stability fell behind competitors' [291], [297]. 
Unlike AMD or Intel processor based systems, the top performing supercomputers using the IBM Power (PC) processor were Many/Multicore systems. IBM initially developed the multicore architecture which later evolved to manycore systems, known as "Blue Gene" technology. The Blue Gene approach is to use a large number of simple processing cores and to connect them via a low latency, highly scalable custom interconnect [298]. This has the advantage of achieving a high aggregate memory bandwidth, whereas GPU clusters require messages to be copied from the GPU to the main memory and then from main memory to the remote node, whilst maintaining low power consumption as well as cost and floor space efficiency [299]. Currently, GPU/Accelerator based systems suggest smaller cluster solutions for the next generation HPC with its promising performance potential; however, the Blue Gene architecture demonstrates an alternate direction of massively parallel quantities of independently operating cores with fewer programming challenges involved [300]. 


\section{c) Model validation}

To validate a predictive performance of the constructed model, hold-out sample tests were conducted. Specifically, a rolling origin was used to determine the forecast accuracy by collecting deviations from multiple forecasting origins so that the performance of the model can be tested both in the near-term and far-term. This provides an objective measure of accuracy without being affected by occurrences unique to a certain fixed origin [261]. The comparative results with the planar model and random walk ${ }^{9}$ are summarized in Table 22.

Since the first hybrid system, the Blade Center QS22, appeared in 2008 in the dataset, the hold-out sample test was conducted from the origin of 2009 for hybrid systems. For example, the mean absolute deviation of 1.58 years was obtained from TFDEA when the model made a forecast on arrivals of post-2009 hybrid systems based on the rate of technological progress observed from 2008 to 2009. The overall forecasting error across the forecasting origins was found to be 1.32 years, which is more accurate than the planar model and random walk.

Although multicore systems showed successive introductions from 2007 to 2012, technological progress, i.e., expansion of SOA frontier surface, hasn't been observed until 2010. This rendered the model able to make a forecast only in 2011. The resulting forecast error of TFDEA was found to be about a year, which is slightly greater than that of the planar model albeit still more accurate than the random walk. However, care must be taken to conclude which one was more accurate than the other since the result was

\footnotetext{
${ }^{9}$ The random walk model simply predicts that the next period value is the same as the current value, i.e., the arrivals of forecasting targets $=$ the forecasting origin [319]. 
obtained only from a single forecasting origin in 2011. Therefore, the forecasting of the multicore Exascale system will be made using both TFDEA and the planar model in the following section.

Consecutive introductions of manycore systems with a steady technological progress made it possible to conduct hold-out sample tests from the origin of 2007 to 2012 . Notwithstanding a bigger average forecasting error of 1.49 years due to the inclusion of errors from longer forecasting windows than the other two systems, TFDEA showed outperforming forecast results compared to the planar model and random walk.

Table 22 Model validation using rolling origin hold-out sample tests

\begin{tabular}{cccc|ccc|ccc}
\hline & \multicolumn{7}{c}{ Mean absolute deviation (unit: year) } \\
\cline { 2 - 10 } $\begin{array}{c}\text { Forecast } \\
\text { Origin }\end{array}$ & \multicolumn{3}{c}{ Hybrid systems } & \multicolumn{3}{c}{ Multicore systems } & \multicolumn{3}{c}{ Manycore systems } \\
\cline { 2 - 11 } & TFDEA & $\begin{array}{c}\text { Planar } \\
\text { model }\end{array}$ & $\begin{array}{c}\text { Random } \\
\text { walk }\end{array}$ & TFDEA & $\begin{array}{c}\text { Planar } \\
\text { model }\end{array}$ & $\begin{array}{c}\text { Random } \\
\text { walk }\end{array}$ & TFDEA & $\begin{array}{c}\text { Planar } \\
\text { model }\end{array}$ & $\begin{array}{c}\text { Random } \\
\text { walk }\end{array}$ \\
\hline 2007 & N/A & N/A & N/A & N/A & N/A & N/A & $\mathbf{1 . 8 0 7 5}$ & 2.8166 & 2.9127 \\
2008 & N/A & N/A & N/A & N/A & N/A & N/A & $\mathbf{1 . 4 4 7 0}$ & 2.5171 & 2.4949 \\
2009 & $\mathbf{1 . 5 8 1 4}$ & 2.7531 & 2.1852 & N/A & N/A & N/A & $\mathbf{2 . 0 0 6 0}$ & 2.3593 & 2.0509 \\
2010 & $\mathbf{1 . 1 1 8 5}$ & 1.9956 & 1.5610 & N/A & N/A & N/A & $\mathbf{1 . 4 9 9 6}$ & 2.0863 & 1.6016 \\
2011 & 1.8304 & $\mathbf{1 . 5 4 1 1}$ & 1.2778 & 0.9899 & $\mathbf{0 . 7 4 9 8}$ & 1.0000 & $\mathbf{1 . 2 7 3 9}$ & 1.8687 & 1.3720 \\
2012 & $\mathbf{0 . 7 5 6 4}$ & 1.2012 & 1.0000 & N/A & N/A & N/A & $\mathbf{0 . 8 8 6 6}$ & 2.2269 & 1.0000 \\
\hline Average & $\mathbf{1 . 3 2 1 7}$ & 1.8728 & 1.5060 & 0.9899 & $\mathbf{0 . 7 4 9 8}$ & 1.0000 & $\mathbf{1 . 4 8 6 7}$ & 2.3125 & 1.9053 \\
\hline
\end{tabular}

Overall, it is shown that the TFDEA model provides a reasonable forecast for three types of supercomputer systems with the maximum possible deviation of 18 months. In 
addition, it is interesting to note that forecasts from TFDEA tended to be less sensitive to the forecasting window than the planar model or random walk.

This implies that the current technological progress of supercomputer technologies exhibits multifaceted characteristics that can be better explained by various tradeoffs derived from the frontier analysis. In addition, a single design tradeoff identified from the planar model was shown to be vulnerable to the forecasting window: it showed a tendency to be less accurate as the forecasting window gets longer. 


\section{d) Forecasting}

I now turn to the forecasting of the Exascale systems. As previously noted, the design goal of the Exascale supercomputer is not only to have the Exaflops $\left(10^{18}\right.$ flop / second) computing performance but also 20MW power consumption and 100 million total cores considering the realistic operating conditions (see Table 23) [276], [280]. This set of specifications was set as a forecasting target to estimate when this level of system could be operational given the past technological progress identified from the relevant segments.

Table 23 Exascale computer as a forecasting target

\begin{tabular}{ccc}
\hline Cores & Power & Rmax \\
\hline 100 million & $20 \mathrm{MW}$ & 1 Exaflops \\
\hline
\end{tabular}

Table 24 summarizes the forecasting results from the three architectural approaches. Exascale performance was forecasted to be achieved earliest by hybrid systems in 2021.13. Hybrid systems are expected to accomplish this with a relatively high individualized RoC of $2.22 \%$ and having the best current level of performance represented by Tianhe-2. Figure 19 depicts the identified individualized RoC with respect to the local RoCs. It is seen that the technology frontier of hybrid systems includes a wide range of progress patterns in terms of local RoCs, i.e., $0.27 \%$ 2.71\%, and the Exascale target is subject to the relatively fast advancing segment.

Considering the possible deviations identified in the previous section $( \pm 1.32$ year $)$, one could expect the arrival of a hybrid Exascale system within the 2020 timeframe. This promising future of hybrid systems is, in fact, acknowledged by many industry experts 
claiming that GPU/Accelerator based systems will be more popular in the TOP500 list for their outstanding power efficiency, which may spur the Exascale development [282], [285].

\begin{tabular}{|l|l|l|l|l|}
\hline Hybrid system & $\mathbf{0 . 2 7 \%}$ & & \\
\hline
\end{tabular}

Figure 19 Individualized RoCs with respect to the local RoCs

The forecasted arrival time of the first multicore based Exascale system is far beyond 2020 due to the slow rate of technological advancement: $1.19 \%$ as well as relatively lower performance of current SOA multicore systems. It is also shown from Fig. 19 that the technology frontier of multicore systems has relatively narrow ranges of local RoCs, i.e., $0.48 \% \sim 1.86 \%$, and, inter alia, the Exascale target is pertinent to the moderate segment.

Note that projection from the planar model also estimated the arrival of multicore based Exascale system farther beyond the 2020 timeframe $^{10}$.

This result implies that innovative engineering efforts are required for multicore based architecture to be scaled up to the Exaflop performance. Even though the RIKEN

\footnotetext{
${ }^{10}$ The arrival of the first multicore Exascale system was forecasted in 2061.62 from the planar model.
} 
embarked on the project to develop the Exascale system continuing the preceding success of K-computer, IBM's cancellation of the Blue Water contract and recent movement toward the use of a design house raises questions on the prospect of multicore based HPCs [301], [302].

The first manycore based system is expected to reach the Exascale target by 2022.28. This technology path has been mostly led by the progress of the Blue Gene architecture, and the individualized $\mathrm{RoC}$ was found to be $2.34 \%$, which was the fastest of the three. It is interesting to note from Fig. 19 that this fast progress, however, belongs to the moderate region of the technology frontier where the local RoCs range from $1.09 \%$ to $3.40 \%$.

Although this fast advancement couldn't overcome the current performance gap with hybrid systems in the Exascale race, the Blue Gene architecture still suggests a promising pathway toward the Exascale computing by virtue of its stable configurations closer to the traditional design with fewer programming challenges [299].

Table 24 Forecast results of Exascale supercomputer

\begin{tabular}{cccc}
\hline & Hybrid system & Multicore system & Manycore system \\
\hline $\begin{array}{c}\text { Individualized } \\
\text { Rate of change (RoC) }\end{array}$ & 1.022183 & 1.011872 & 1.023437 \\
\hline $\begin{array}{c}\text { Forecasted arrival of } \\
\text { Exascale supercomputer }\end{array}$ & $(2019.81 \sim 2022.45)$ & $(2030.75 \sim 2032.73)$ & $(2020.80 \sim 2023.77)$ \\
\hline
\end{tabular}

( ): Ranges of forecasts considering the possible deviations 


\section{DISCUSSION}

The analysis of technological RoC makes it possible to forecast a date for achieving Exascale performance from three different approaches; however, it is worthwhile to examine these forecasts with consideration for the business environment and emerging technologies to anticipate the actual deployment possibilities of the Exascale systems.

The optimistic forecast is that, as seen from the high performing Tianhe- 2 and Titan Cray XK7 system, there would be an Intel or AMD based system with a Xeon Phi or Nvidia coprocessor and a custom Cray interconnect system. However, given business realities it is unlikely that the first Exascale system will use AMD processors. Intel purchased the Cray interconnect division and is expected to design the next generation Cray interconnect optimized for Intel processors and Xeon Phi coprocessors [303]. Existing technology trends and the changing business environment would make a forecast of a hybrid Exascale system with a Cray interconnect, Intel Processors and Xeon Phi coprocessors.

The $2.22 \%$ annual improvement for hybrid systems has come mostly from a combination of advances in Cray systems, such as their transverse cooling system, Cray interconnects, AMD processors and Nvidia coprocessors. It is difficult to determine the contribution of each component; however, it is worth noting that only Cray systems using AMD processors were SOAs. This implies that Cray's improvements are the highest contributor to the RoC for AMD based hybrid systems. Furthermore, Intel's recent decision to move production of Cray interconnect chips from TSMC to its more advanced processes will likely result in additional performance improvement. Thus, one may 
expect that the Cray / Intel collaboration might result in a RoC greater than the $2.22 \%$ and reach the Exascale goal earlier.

Another possibility of achieving Exascale systems is IBM's Blue Gene architecture using the IBM Power (PC) processor with custom interconnects. This approach has shown a $2.34 \%$ yearly improvement building on the 3rd highest rated Sequoia system. The Blue Gene architecture, with high bandwidth, low latency interconnects and no coprocessors to consume bandwidth or complicate programming, is an alternative to the coprocessor (hybrid) architectures being driven by Intel and AMD. Given IBM's more stable business structure, it may be more effective moving forward while Intel / Cray work out their new relationship.

Who has the system experience to build an Exascale system? Cray, IBM and Appro have built the largest SOA original equipment manufacturer (OEM) systems. In 2012, Cray purchased Appro, leaving just two major supercomputer manufacturers [304]. Based upon the captured RoCs and the business changes, one can expect that the first Exascale system will be built by either Cray or IBM.

As supercomputer systems become more complex and expensive, it is worth noting the funding efforts in a story about the future of HPC. The U.S. Department of Energy (DOE) recently awarded $\$ 425$ million in federal funding to IBM, Nvidia, and other companies that will build two 150 Petaflops systems with an option on one system to build it out to 300 Petaflops [305]. The plan states that IBM will supply its Power architecture processors, while Nvidia will supply its Volta GPUs, and Mellanox will provide interconnected technologies to wire everything together [306]. In addition, the DOE and the National Nuclear Security Administration (NNSA) have announced $\$ 100$ 115 
million to fund the FastForward2 project that will develop technologies needed for future energy efficient machines in collaboration with AMD, Cray, IBM, Nvidia and Intel [307]. One may notice that U.S. science funding will support both hybrid and manycore systems for producing the next leap toward the Exascale.

Japan had earlier announced a goal to reach Exascale with the total project cost of $\$ 1.2$ billion by 2020 [308]. However, the deputy director at the RIKEN Advanced Institute for Computational Science (RAICS) recently modified the goal and plans to build a 200 to 600 Petaflops system by 2020. Nonetheless, given the fact that RIKEN selected Fujitsu to develop the basic design for the system, there is a keen interest in how much the multicore system could be scaled up with a relatively low power efficiency of complex cores.

Data driven forecasting techniques, such as TFDEA, make a forecast of technical capabilities based upon released products, so emerging technologies that are not yet being integrated into products are not considered. In the supercomputer academic literature, there is an ongoing debate about when the currently dominating large core processors (Intel, AMD) will be displaced by larger numbers of power-efficient, lower performance small cores such as ARM, much like what happened when microprocessors displaced vector machines in the 1990's and ARM based mobile computing platforms are affecting both Intel and AMD desktop and laptop sales [282], [287]. Although there is no ARM based supercomputer in the TOP500 yet, the European Mont-Blanc project is targeting getting one on the list by 2017, and Nvidia is developing an ARM based supercomputer processor for use with its coprocessor chips [309]. Small cores are a potentially disruptive technology as power efficiency is becoming more important; therefore, further analysis is 
needed to investigate when it will overcome the challenges of building interconnects to handle a larger number of smaller cores or when software developers will overcome the synchronization challenges of effectively using more cores.

Another new kind of supercomputer attracting recent attention is the superconducting supercomputer (as one way to enable the quantum computing). Even though the exact financial and technical details with a timeframe were not disclosed, the Intelligence Advanced Research Projects Activity (IARPA) revealed that funding contracts have been awarded to IBM, Raytheon-BBN and Northrop Grumman Corporation focusing on the development of the Cryogenic Computer Complexity (C3) program [310]. Early research suggested that a superconducting supercomputer would be able to provide around 100 Petaflops of performance while consuming just 200 kilowatts [311]. If the mission of the C3 program can be achieved and the related technologies can be successfully transferred to practical usages, the next generation supercomputers could be far different from the ones of the past, and the Exascale goal could be achieved without concerns of power and cooling capacities.

Lastly, this study set the Exascale target considering the realistic operating conditions: 20MW of power consumption and 100 million cores. If this set of specifications was relaxed at the manufacturers' free will, the arrival of an Exascale computer could come earlier than current forecasts as China is believed to be targeting the 2018-2020 timeframe for continuing their gigantic design of Tianhe-2. 


\section{SUMMARY OF FINDINGS}

The HPC industry is experiencing a radical transition which requires improvement of power efficiency by a factor of 23 to deploy and/or manage the Exascale systems. This has created an industry concern that the naïve forecast based on the past performance curve may have to be adjusted. TFDEA is well suited to deal with multiple tradeoffs between systems attributes. This study examined comparative prospects of three competing technology alternatives with various design possibilities considering the complex business environment to achieve the Exascale computing so that researchers and manufacturers can have a better view of their development targets. In sum, the results showed that the current development target of 2020 might entail technical risks considering the rate of change toward the power efficiency observed in the past. It is anticipated that either a Cray built hybrid system using Intel processors or an IBM built Blue Gene architecture system using PowerPC processors will likely achieve the goal between early 2021 and late 2022 .

In addition, the results provided a systematic measure of technological change, which can guide a decision on the new product target setting practice. Specifically, the rate of change contains information not only about how much performance improvement is expected to be competitive but also about how much technical capability should be relinquished to achieve a specific level of technical capabilities in other attributes. One can also utilize this information to anticipate the possible disruptions. As shown in the HPC industry, the rate of change of the manycore system was found to be slightly faster than that of the hybrid system. Although the arrival of the hybrid Exascale system is forecasted earlier than a manycore system because of its current surpassing level of 
performance, the fast rate of change of the manycore system implies that the performance gap could be overcome, and Blue Gene architecture might accomplish the Exascale goal earlier if the hybrid system development is unable to keep up with the expected progress. 


\section{CONCLUSION}

\subsection{Contributions to Application Area}

\subsubsection{EXASCALE SUPERCOMPUTER DEVELOPMENT}

Experts and recent literature commonly point out that technological progress of supercomputers needs to be re-examined since power consumption and effective parallelism are increasingly critical factors to build Exascale systems. Above all, improvement of power efficiency by a factor of 23 is required to deploy and operate the Exascale systems in practice. This unprecedented type of technology necessarily requires choosing a high-level implementation strategy with respect to the tradeoffs between system attributes. There are three leading architectures-hybrid, multicore, and manycore systems - to cope with related challenges, and manufacturers and researchers are exploring their expected potentials to have an accurate view of the technological advancement in high performance computing.

The newly developed extensions to TFDEA are well suited to this application to deal with multiple tradeoffs between systems attributes. The results provide comparative prospects of those three competing technology alternatives with various design possibilities accounting for the business environment to achieve the Exascale systems. Specifically, the results indicate that the first Exascale system is likely to be built by Cray or IBM with their customized interconnects. Considering the recent business changes, it is more likely that the first Exascale performance can be achieved by Intel processors and Xeon Phi coprocessors as hybrid systems. 
In addition, the identified rates of change can be used to give insights into the estimation of the future performance levels for new product development target setting purposes. Supercomputer manufacturers may have their own roadmaps based on past performance improvement, which has been mostly driven by the computation speed. However, as noted above, the transition toward Exascale demands considering both power and core efficiency. This would necessarily require the established roadmap to be modified. There are three alternatives, i.e., hybrid, multicore, and manycore systems, each heading toward the same goal. Who then do manufacturers bet on to win the race? In addition, how much performance improvement should be made by a certain point in time to meet the planned delivery of the Exascale computer? The rate of change contains information to better inform their decisions.

One can also utilize rates of change to anticipate the possible disruptions. For example, the rate of change of manycore systems was found to be faster than that of hybrid system. Although the arrival of a hybrid Exascale system is forecasted earlier because of its current level of performance being superior to manycore systems, this indicates that the performance gap could be overcome, and the Blue Gene architecture might accomplish the Exascale goal earlier within the possible forecast deviation if Cray and Intel are unable to keep up with performance advancement expected from the given rate of change while they work out their new relationship. 


\subsection{CONTRIBUTIONS TO MANAGERIAL INSIGHT}

\subsubsection{RISK ANALYSIS}

One of the motivations of this study stemmed from a practical question: "When might be the ideal timing to release the new product?" To answer this question, one may have to know not only what segments the current product concept is subject to but also how competitive corresponding segments are. This subject can be translated into the research topic of integrating product positioning with the assessment of performance improvement over time, which has rarely been addressed in both new product development and management science literature. The presented use of time series benchmarking analysis makes it possible to estimate the "auspicious" time by which proposed design concepts will be operational as competitive products by taking into account the rate of performance improvement expected in a target segment.

The empirical illustration of commercial airplane development has shown that the new method provides valuable information such as dominating designs, distinct segments, and the potential rate of performance improvement, which can be utilized in the early stage of new product development. In particular, six SOA airplanes characterizing distinct segments were identified with corresponding local RoCs and, inter alia, technological advancement toward long-range and wide-body airplanes represented very competitive segments of the market with rapid changes. The resulting individualized RoCs were able to estimate the arrivals of four different design concepts, which is consistent with what has happened since 2007 in the commercial airplane industry.

Similarly, the case study of Exascale supercomputer development showed that the current development target of 2020 might entail technical risks considering the rate of 
change toward the power efficiency observed in the past. It is forecasted that either a Cray built hybrid system using Intel processors or an IBM built Blue Gene architecture system using PowerPC processors will likely achieve the goal between early 2021 and late 2022. This indicates that the improvement of power efficiency by a factor of 23 would require the maximum delay of four years from the past performance curve.

\subsubsection{NEW PRODUCT TARGET SETTING}

Unlike market research methods or heuristic ideation techniques, this study employs a product feature-based clustering approach. This engineering approach can be used to identify homogeneous product groups by ordering products from broad to niche based on the number of times each product appears in others' reference sets. This information can be very useful for decision makers to position their products by referring to product designs in other clusters, which provides a direction to adjust the combination of its product attributes so as to be assigned into a desired cluster.

The rate of technological advancement identified in each market segment can further give insights into the target setting practices for a new product development planning. That is, manufacturers may position their products within the current SOA frontier and utilize the corresponding rate of change to see whether their design targets would be located on the estimated future frontiers. Similarly, one could also utilize this information for the pricing strategy if the price was included as an input variable. 


\subsection{Contributions to Methodological Development}

\subsubsection{IDENTIFICATION OF LOCAL RATE OF CHANGE}

As an answer to the first research question, a new model was developed to capture the local RoCs from homogeneous technologies in the past. This enables each SOA technology not only to characterize the corresponding segments but also to represent the unique progress patterns. The resulting segmented rate of change extensions to TFDEA is an objective data-driven process.

Table 25 Answer to research question 1

Research

Question

\#1

Answer

to

Research

Question

\#1
How do we capture the local rate of change from past technologies?

The efficiency changes of past products with respect to corresponding segments are aggregated by taking the weighted average using the reference information. Each local RoC therefore represents a growth potential of adjacent frontier facets based on the technological advancement observed from related past products.

\subsubsection{IDENTIFICATION OF INDIVIDUALIZED RATE OF CHANGE}

As an answer to the second research question, a new model was developed to identify the appropriate progress potential, i.e., individualized $\mathrm{RoC}$, for each forecasting target. Similar to the process of calculating local RoCs from surpassed technologies in the past, individualized RoCs can be obtained from SOA technologies that present similar operating practices. Seven rolling-origin hold-out sample tests have shown that this approach improves the overall forecasting results in comparison to the original TFDEA model. 
Table 26 Answer to research question 2

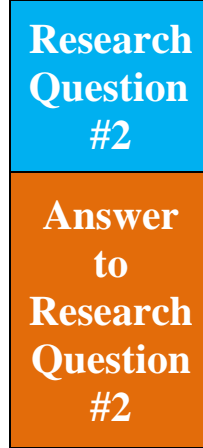

How do we determine the individualized rate of change for future technologies?

Reference information of each forecasting target is used to combine relevant local RoC(s) to identify an individualized RoC under which each forecasting target is expected to arrive.

\subsubsection{A FINITE FORECAST FOR AN INFEASIBLE TARGET}

As an answer to the third research question, a new model was developed to make a finite forecast for formerly infeasible targets. Specifically, this model identifies bidirectional distances, i.e., extremity and standard radial distance, for infeasible targets, and therefore TFDEA computes both input-oriented and output-oriented RoCs and applies them to estimate the arrival of target technologies. By virtue of the adopted alternate super-efficiency measure, this approach yields the forecast results identical to those from the original TFDEA model when the target is feasible. Consequently, a new model can be fully integrated as an extension of the TFDEA model.

Table 27 Answer to research question 3

Research

Question

\#3

Answer

to

Research

Question

\#3
How do we deal with infeasible targets?

Bi-directional L1 distances are obtained and time spans for each distance are estimated using corresponding RoCs, i.e., current orientation RoC for standard radial distance and the opposite orientation RoC for extremity (if any), from both orientations. 


\subsection{LIMITATIONS}

This study adopts an engineering design perspective that a product is a complex assembly of interacting components. Consequently, the term "segment" is being used to indicate a set of engineering designs having a similar mix of product attributes. Note that the academic community in marketing uses this term with a broader implication of shared needs and value propositions determined by a meaningful number of customers. In this study, the identified targets and competitors are derived purely based on technical specifications. This attribute-based approach can be limited in its ability to represent the overall appeal of products especially those for which other holistic product features that are not reflected in technical specifications such as aesthetics are important.

In a similar vein, the DEA measurement is based on the relative performance of the products, and therefore the state-of-the-art products may be the most advanced ones but may not be the most successful ones in the market. This indicates that the resulting rate of change is more likely to be reflected by the technological progress than market desires.

In addition, the estimation of release date from the proposed model doesn't take into account externalities such as strategic postponement, financial conditions, market acceptance levels, self-imposed delay due to the product portfolio management, etc. Therefore, the resulting release date should be understood as a baseline for implementing the tactical launch decision with respect to product attributes concerned rather than the bottom line of decision. This also suggests that the estimated release date may have to be

further adjusted if the industrial market is less sensitive to the technological superiority than to market strategies. 


\subsection{FUTURE WORK DIRECTIONS}

This section suggests future work directions including methodological extensions of proposed model as well as its applications to the managerial decision makings.

\subsubsection{INNOVATIVENESS MEASURE}

One might make use of a presented risk analysis approach to develop a measure of innovativeness. When there is a need to quantify the innovativeness of products independent from market factors, this method can suggest how much a certain product has contributed to accelerating the rate of performance improvement or has moved up the product release compared to the expected arrival. Alternate approaches could also investigate the possible modifications in product designs to reduce the risk, thereby meeting the release date determined.

\subsubsection{ALTERNATE EFFICIENCY MEASURES}

A DEA measure is by definition an equiproportional ratio of how the DMU being assessed can either reduce its inputs or augment its outputs to reach its virtual target. This radial efficiency score may not account for all sources of inefficiency by having input and/or output slacks that are not reflected in the collective proportion. Further, the traditional DEA model is labeled as "radial" since it gives preemptive priority either to conservation of the input or to expansion of the output, depending on model orientation. This implies that the radial approach may not capture the technological advancement within structural characteristics or functional improvements, while the technology systems' objective might often be the desire to change the mix of them. This suggests the 
non-radial and/or non-oriented distance measures for estimating the frontier with consideration of the furthest target, closest target, restricted targets, scale-efficient target, or target located in a predefined direction that could set more realistic targets whereby diverse patterns of technological advancement can be explored.

Table 28 Alternate non-radial efficiency measures

\begin{tabular}{lll}
\hline \multicolumn{2}{c}{ Oriented measure } & \multicolumn{1}{c}{ Non-oriented measure } \\
\hline$\bullet \quad$ Russell measure & $\bullet$ & Additive model \\
- Geometric distance function model (GDF) & $\bullet \quad$ Range-adjusted model (RAM) \\
- Hyperbolic model & $\bullet \quad$ Slack-based model (SBM) \\
& $\bullet \quad$ Proportional slack-based model (P-SBM) \\
& $\bullet$ & Directional model \\
\hline
\end{tabular}

\subsubsection{WEIGHT RESTRICTIONS}

Another future research topic might consider exploring the product segmentation by imposing weight restrictions on the model. The current model doesn't require an axiomatic weighting scheme on any attributes being considered. This might cause an overestimation of products having an extreme feature in certain attribute(s). To avoid this, one can establish the boundary of weights attached to certain attributes whereby significant value propositions of both extant and potential market segments can be reflected into the analysis. This includes determining how much certain attributes should be valued than others as well as how much maximum (or minimum) weight can be assigned to certain attributes. 


\subsubsection{TIME VARYING RATE OF CHANGE}

Another direction of future work could also investigate the varying RoCs over time. The local RoCs can provide information about the number of distinct segments within which differing rates of technological advancement have been captured. One can examine this information in conjunction with maturity curves so that market dynamics among identified segments can be studied.

\subsubsection{DireCtion OF TECHNOLOGICAL PROGRESS}

Comparative results between the input-oriented model and the output-oriented model could provide insights regarding whether the direction of innovation has been switched. As previously discussed, an infeasible target in the input (output)-oriented model may occur when the output (input) levels of the target technology are unprecedented in the past. Hence, one might derive an indication of technological progress from an increasing/decreasing number of infeasible targets over time. In a similar manner, the magnitude of extremity may contain relevant information about the potential paths of technological progress.

\subsubsection{STOCHASTIC FRONTIER}

Lastly, incorporating stochastic variation into the model must be a matter for speculation. DEA is by definition a deterministic model which renders the presented method confined to capturing the rate of performance improvement from the evolution of the SOA frontier. This might provide an aggressive estimation unless the best performing products on the market are sought. Hence, stochastic measurements would be able to 
complement this aspect such that the rate of performance improvement can be obtained from diverse levels of products, thereby yielding the risk distribution for each design concept instead of a point estimation. 


\section{References}

[1] N. Taleb, The Black Swan: The Impact of the Highly Improbable Fragility. New York: Random House LLC, 2010.

[2] R. MacLean, "The six mistakes executives make in risk management," Courtesy of Richard MacLean \& Associates, LLC, 2010.

[3] E. Jantsch, Technological Forecasting in Perspective. Paris: OECD, 1967, p. 401.

[4] P. Saffo, "Six rules for effective forecasting," Harv. Bus. Rev., vol. 85, no. 7/8, pp. 122-131, 2007.

[5] R. Lenz, Technological Forecasting, 2nd ed. Virginia: Defense Documentation Center for Scientific and Technical Information, 1962, p. 107.

[6] A. L. Porter, S. W. Cunningham, J. Banks, A. T. Roper, and T. W. Mason, Forecasting and Management of Technology, 2nd ed., vol. 18. John Wiley \& Sons, 2011, p. 336.

[7] D.-J. Lim, S. R. Jahromi, T. R. Anderson, and A. A. Tudorie, "Comparing technological advancement of hybrid electric vehicles (HEV) in different market segments," Technol. Forecast. Soc. Change (In press), 2015.

[8] N. D. Kondratiev, "The major economic cycles," Vopr. Konjunktury, vol. 1, no. 1, pp. 28-79, 1925.

[9] J. A. Schumpeter, "Business cycles, migration and health.," Soc. Sci. Med., vol. 64, no. 7, pp. 1420-4, 1939.

[10] R. N. Foster, Innovation: The Attacker's Advantage. Summit Books, 1986, p. 316.

[11] J. M. Utterback, Mastering the Dynamics of Innovation : How Companies can Seize Opportunities in the Face of Technological Change, vol. 81, no. 1. Harvard Business School Press, 1994, pp. 138-140.

[12] G. Yokote and R. A. Utterback, "Time lapses in information dissemination: research laboratory to physician's office," Bull. Med. Libr. Assoc., vol. 62, no. 3, pp. 251-257, 1974.

[13] A. Sood and G. J. Tellis, "Technological evolution and radical innovation," J. Mark., vol. 69, no. 3, pp. 152-168, 2005. 
[14] D. Sahal, "The farm tractor and the nature of technological innovation," Res. Policy, vol. 10, no. 4, pp. 368-402, 1981.

[15] C. Christensen, The Innovator's Dilemma, vol. 1, no. 1. Harvard Business School Press, 1997, p. 225.

[16] E. Danneels, "Disruptive technology reconsidered: a critique and research agenda," J. Prod. Innov. Manag., vol. 21, no. 4, pp. 246-258, Jul. 2004.

[17] D. Yu and C. C. Hang, "A reflective review of disruptive innovation theory," Int. J. Manag. Rev., vol. 12, no. 4, pp. 435-452, Dec. 2010.

[18] G. J. Tellis, "Disruptive technology or visionary leadership?," J. Prod. Innov. Manag., vol. 23, no. 1, pp. 34-38, Jan. 2006.

[19] P. S. Cohan, "The dilemma of the "Innovator"s Dilemma": Clayton Christensen's management theories are suddenly all the rage, but are they ripe for disruption?," Industry Standard, vol. 10, Industry Standard, 2010.

[20] C. M. Christensen and M. Overdorf, "Meeting the challenge of disruptive change," Harv. Bus. Rev., vol. 78, no. 2, pp. 66-76, 2000.

[21] J. R. Doyle and R. H. Green, "Comparing products using data envelopment analysis," Omega, vol. 19, no. 6, pp. 631-8, 1991.

[22] L. M. Seiford and J. Zhu, "Context-dependent data envelopment analysisMeasuring attractiveness and progress," Omega, vol. 31, no. 5, pp. 397-408, 2003.

[23] R.-W. Po, Y.-Y. Guh, and M.-S. Yang, "A new clustering approach using data envelopment analysis," Eur. J. Oper. Res., vol. 199, no. 1, pp. 276-284, 2009.

[24] A. Amirteimoori and S. Kordrostami, "An alternative clustering approach: a DEAbased procedure," Optimization. pp. 1-14, 2011.

[25] X. Dai and T. Kuosmanen, "Best-practice benchmarking using clustering methods: Application to energy regulation," Omega, vol. 42, no. 1, pp. 179-188, 2014.

[26] P. Bogetoft and L. Otto, Benchmarking with DEA, SFA, and R, 1st ed. New York: Springer, 2010, p. 367.

[27] L. M. Seiford and J. Zhu, "Infeasibility of super-efficiency data envelopment analysis models," INFOR, vol. 37, no. 2, pp. 174-188, 1999. 
[28] T. Modis, "Strengths and weaknesses of S-curves," Technol. Forecast. Soc. Change, vol. 74, no. 6, pp. 866-872, Jul. 2007.

[29] T. S. Kuhn, The Structure of Scientific Revolutions, vol. 57, no. 1. Chicago, IL : University of Chicago Press, 1996. 3rd ed., 1996, pp. 59-75.

[30] H. I. Weisberg, Bias and Causation: Models and Judgment for Valid Comparisons, 1st ed. Needham: Wiley, 2010, pp. 1-348.

[31] J. M. Utterback, "Radical innovation and corporate regeneration," Res. Manag., vol. 37, no. 4, p. 10, 1994.

[32] T. W. Danner, "A formulation of multidimensional growth models for the assessment and forecast of technology attributes," Georgia Institute of Technology, 2006.

[33] C. Christensen, "Exploring the limits of the technology S-curve. Part I: Component technologies," Prod. Oper. Manag., vol. 1, no. 4, pp. 334-357, 2009.

[34] M. A. Schilling and M. Esmundo, "Technology s-curves in renewable energy alternatives: analysis and implications for industry and government," Energy Policy, vol. 37, no. 5, pp. 1767-1781, 2009.

[35] C. M. Christensen, "A history of the rigid disk drive industry : commercial and technological turbulence,” Bus. Hist., vol. 67, no. 4, pp. 531-588, 1993.

[36] A. Debecker and T. Modis, "Determination of the uncertainties in S-curve logistic fits," Technol. Forecast. Soc. Change, vol. 46, no. 2, pp. 153-173, 1994.

[37] P. Young, "Technological growth curves: a competition of forecasting models," Technol. Forecast. Soc. Change, vol. 44, no. 4, pp. 375-389, 1993.

[38] J. P. Martino, Technological Forecasting for Decision Making, 2nd ed. NorthHolland: McGraw-Hill, 1993.

[39] R. Fildes, "An evaluation of bayesian forecasting," J. Forecast., vol. 2, no. 2, pp. 137-150, 1983.

[40] S. Makridakis, A. Anderson, and R. Carbone, The Forecasting Accuracy of Major Time Series Methods. Chichester (Wiley), 1984, p. 301.

[41] P. Young and J. Keith Ord, "The use of discounted least squares in technological forecasting,” Technol. Forecast. Soc. Change, vol. 28, no. 3, pp. 263-274, 1985. 
[42] R. L. Chadda and S. S. Chitgopekar, "A generalization of the logistic curves and long-range forecasts (1966-1991) of residence telephones," Bell J. Econ. Manag. Sci., vol. 2, pp. 542-560, 1971.

[43] S. Makridakis, S. C. Wheelwright, and V. E. McGee, Forecasting: Methods and Applications, Second. New York: John Wiley \& Sons, 1983, p. 923.

[44] E. Mansfield, "Technological change and the rate of imitation," Econometrica, vol. 29, no. 4, pp. 747-766, 1961.

[45] M. N. Sharif and M. N. Islam, "The Weibull distribution as a general model for forecasting technological change," Technol. Forecast. Soc. Change, vol. 18, no. 3, pp. 247-256, 1980.

[46] F. M. Bass, "A new product growth for model consumer durables," Manage. Sci., vol. 15 , no. 5, pp. 215-227, 1969.

[47] C. Easingwood, V. Mahajan, and E. Muller, "A nonsymmetric responding logistic model for forecasting technological substitution," Technol. Forecast. Soc. Change, vol. 20, no. 3, pp. 199-213, 1981.

[48] A. C. Harvey, “Time series forecasting,” J. Oper. Res. Soc., vol. 35, pp. 641-646, 1984.

[49] H. Levenbach and B. E. Reuter, "Forecasting trending time series with relative growth rate models," Technometrics, vol. 18, no. 3, pp. 261-272, 1976.

[50] J. P. Martino, "A review of selected recent advances in technological forecasting," Technol. Forecast. Soc. Change, vol. 70, no. 8, pp. 719-733, 2003.

[51] R. U. Ayres, "Toward a non-linear dynamics of technological progress," J. Econ. Behav. Organ., vol. 24, no. 1, pp. 35-69, 1994.

[52] C. M. Christensen and J. L. Bower, "Customer power, strategic investment, and the failure of leading firms," Strateg. Manag. J., vol. 17, no. 3, pp. 197-218, 1996.

[53] C. M. Christensen and M. E. Raynor, The Innovator's Solution, vol. 25, no. 11. Harvard Business School Press, 2003, p. 304.

[54] H. Chesbrough, "Assembling the elephant: A review of empirical studies on the impact of technical change upon incumbent firms," Comp. Stud. Technol. Evol., vol. 7, pp. 1-36, 2001. 
[55] A. C. Cooper and D. Schendel, "Strategic responses to technological threats," Bus. Horiz., vol. 19, no. 1, pp. 61-69, 1976.

[56] M. L. Tushman and P. Anderson, "Technological discontinuities and organizational environments," Adm. Sci. Q., vol. 31, no. 3, pp. 439-465, 1986.

[57] R. M. Henderson and K. B. Clark, "Architectural innovation: the reconfiguration of existing product technologies and the failure of established firms," Adm. Sci. Q., vol. 35, no. 1, pp. 9-30, 1990.

[58] R. Henderson, "Underinvestment and Incompetence as Responses to Radical Innovation: Evidence from the Photolithographic Alignment Equipment Industry," RAND J. Econ., vol. 24, no. 2, p. 248, 1993.

[59] H. Chesbrough, "Arrested development: the experience of European hard disk drive firms in comparison with US and Japanese firms," J. Evol. Econ., vol. 9, no. 3, pp. 287-329, 1999.

[60] L. H. Lynn, "From silicon valley to singapore: location and competitive advantage in the hard disk drive industry," J. Eng. Technol. Manag., vol. 19, no. 2, pp. $217-$ $219,2002$.

[61] M. Tripsas and G. Gavetti, "Capabilities, cognition, and inertia: evidence from digital imaging," Strateg. Manag. J., vol. 21, no. 10-11, pp. 1147-1161, 2000.

[62] C. E. Helfat and M. B. Lieberman, "The birth of capabilities: market entry and the importance of pre-history," Ind. Corp. Chang., vol. 11, no. 4, pp. 725-760, 2002.

[63] A. A. King and C. L. Tucci, "Incumbent entry into new market niches: the role of experience and managerial choice in the creation of dynamic capabilities," Manage. Sci., vol. 48, no. 2, pp. 171-186, 2002.

[64] S. Klepper and K. L. Simons, "Dominance by birthright: entry of prior radio producers and competitive ramifications in the U.S. television receiver industry," Strateg. Manag. J., vol. 21, no. 10-11, pp. 997-1016, 2000.

[65] R. K. Chandy and G. J. Tellis, "The incumbent's curse? incumbency, size, and radical product innovation," J. Mark., vol. 64, no. 3, pp. 1-17, 2000.

[66] M. Tripsas, "Unraveling the process of creative destruction: complementary assets and incumbent survival in the typesetter industry," Strateg. Manag. J., vol. 18, no. S1, pp. 119-142, 1997. 
[67] F. T. Rothaermel, "Incumbent's advantage through exploiting complementary assets via interfirm cooperation," Strateg. Manag. J., vol. 22, no. 6-7, pp. 687-699, 2001.

[68] M. R. Darby, L. G. Zucker, and A. B. A. H. C. Robert, "Change or die: the adoption of biotechnology in the Japanese and U.S. pharmaceutical industries," Comp. Stud. Technol. Evol., vol. 7, pp. 85-125, 2001.

[69] G. M. Schmidt, "Low-end and high-end encroachment strategies for new products," Int. J. Innov. Manag., vol. 8, no. 2, pp. 167-191, Nov. 2011.

[70] A. Martinelli, “An emerging paradigm or just another trajectory? Understanding the nature of technological changes using engineering heuristics in the telecommunications switching industry," Res. Policy, vol. 41, no. 2, pp. 414-429, 2012.

[71] S. Kassicieh and N. Rahal, "A model for disruptive technology forecasting in strategic regional economic development," Technol. Forecast. Soc. Change, vol. 74, no. 9, pp. 1718-1732, 2007.

[72] R. Phaal, E. O'Sullivan, M. Routley, S. Ford, and D. Probert, "A framework for mapping industrial emergence," Technol. Forecast. Soc. Change, vol. 78, no. 2, pp. 217-230, 2011.

[73] A. Keller and S. Hüsig, "Ex ante identification of disruptive innovations in the software industry applied to web applications: The case of Microsoft's vs. Google's office applications," Technol. Forecast. Soc. Change, vol. 76, no. 8, pp. 1044-1054, 2009.

[74] D. Barberá-Tomás and D. Consoli, "Whatever works: uncertainty and technological hybrids in medical innovation," Technol. Forecast. Soc. Change, vol. 79, no. 5, pp. 932-948, 2012.

[75] S. Husig, C. Hipp, and M. Dowling, "Analysing disruptive potential: the case of wireless local area network and mobile communications network companies," R\&D Manag., vol. 35, no. 1, pp. 17-35, 2005.

[76] K. Adamson, "Calculating the price trajectory of adoption of fuel cell vehicles," Int. J. Hydrogen Energy, vol. 30, no. 4, pp. 341-350, 2005.

[77] R. Letchumanan and F. Kodama, "Reconciling the conflict between the 'pollutionhaven' hypothesis and an emerging trajectory of international technology transfer," Res. Policy, vol. 29, no. 1, pp. 59-79, 2000. 
[78] H. Koh and C. L. Magee, "A functional approach for studying technological progress: Application to information technology," Technol. Forecast. Soc. Change, vol. 73, no. 9, pp. 1061-1083, 2006.

[79] J. Paap and R. Katz, "Anticipating disruptive innovation," IEEE Eng. Manag. Rev., vol. 32, no. 4, pp. 13-22, 2004.

[80] V. Govindarajan and P. K. Kopalle, "The usefulness of measuring disruptiveness of innovations ex post in making ex ante predictions," J. Prod. Innov. Manag., vol. 23, no. 1, pp. 12-18, 2006.

[81] D. S. Doering and P. Roch, "Identification and assessment of emerging technologies," in Wharton on Managing Emerging Technologies, New York: Wiley, 2000, p. 480.

[82] S. T. Walsh, "Roadmapping a disruptive technology: A case study," Technol. Forecast. Soc. Change, vol. 71, no. 1-2, pp. 161-185, 2004.

[83] D. Padgett and M. S. Mulvey, "Differentiation Via Technology: Strategic Positioning of Services Following the Introduction of Disruptive Technology," $J$. Retail., vol. 83, no. 4, pp. 375-391, 2007.

[84] X. Huang and G. Sošić, "Analysis of industry equilibria in models with sustaining and disruptive technology," Eur. J. Oper. Res., vol. 207, no. 1, pp. 238-248, 2010.

[85] D. C. Kaslow, "A potential disruptive technology in vaccine development: genebased vaccines and their application to infectious diseases.," Trans. R. Soc. Trop. Med. Hyg., vol. 98, no. 10, pp. 593-601, 2004.

[86] B. Rao, B. Angelov, and O. Nov, "Fusion of disruptive technologies:: lessons from the Skype case," Eur. Manag. J., vol. 24, no. 2-3, pp. 174-188, 2006.

[87] W. G. Bradley, "MR-guided focused ultrasound: a potentially disruptive technology.," J. Am. Coll. Radiol., vol. 6, no. 7, pp. 510-3, 2009.

[88] H. C. Lucas and J. M. Goh, "Disruptive technology: How Kodak missed the digital photography revolution,” J. Strateg. Inf. Syst., vol. 18, no. 1, pp. 46-55, 2009.

[89] F. Madjdi and S. Hüsig, "The response strategies of incumbent mobile network operators on the disruptive potential of public W-LAN in Germany," Telecomm. Policy, vol. 35, no. 6, pp. 555-567, 2011.

[90] S. T. Walsh, R. L. Boylan, C. McDermott, and A. Paulson, "The semiconductor silicon industry roadmap: Epochs driven by the dynamics between disruptive 
technologies and core competencies," Technol. Forecast. Soc. Change, vol. 72, no. 2, pp. 213-236, 2005.

[91] P. N. Figueiredo, "Discontinuous innovation capability accumulation in latecomer natural resource-processing firms," Technol. Forecast. Soc. Change, vol. 77, no. 7, pp. 1090-1108, 2010.

[92] J. P. Caulkins, G. Feichtinger, D. Grass, R. F. Hartl, and P. M. Kort, "Two state capital accumulation with heterogenous products: Disruptive vs. non-disruptive goods," J. Econ. Dyn. Control, vol. 35, no. 4, pp. 462-478, 2011.

[93] M.-C. Belis-Bergouignan, V. Oltra, and M. Saint Jean, "Trajectories towards clean technology: example of volatile organic compound emission reductions," Ecol. Econ., vol. 48, no. 2, pp. 201-220, 2004.

[94] J. C. Ho, "Technology evaluation in Taiwan's technology industries: Strategies, trajectories, and innovations," Technol. Forecast. Soc. Change, vol. 78, no. 8, pp. 1379-1388, 2011.

[95] S. H. Werfel and A. B. Jaffe, "Induced innovation and technology trajectory: Evidence from smoking cessation products," Res. Policy, vol. 42, no. 1, pp. 15-22, 2012.

[96] H. J. No and Y. Park, "Trajectory patterns of technology fusion: Trend analysis and taxonomical grouping in nanobiotechnology," Technol. Forecast. Soc. Change, vol. 77, no. 1, pp. 63-75, 2010.

[97] Y. E. Spanos and I. Voudouris, "Antecedents and trajectories of AMT adoption: The case of Greek manufacturing SMEs," Res. Policy, vol. 38, no. 1, pp. 144-155, 2009.

[98] K. Frenken and L. Leydesdorff, "Scaling trajectories in civil aircraft (1913-1997)," Res. Policy, vol. 29, no. 3, pp. 331-348, 2000.

[99] C. Watanabe, S. Lei, and N. Ouchi, "Fusing indigenous technology development and market learning for greater functionality development-An empirical analysis of the growth trajectory of Canon printers," Technovation, vol. 29, no. 4, pp. 265283, 2009.

[100] M. Hobo, C. Watanabe, and C. Chen, "Double spiral trajectory between retail, manufacturing and customers leads a way to service oriented manufacturing," Technovation, vol. 26, no. 7, pp. 873-890, 2006. 
[101] C. Watanabe, J. Y. Hur, and K. Matsumoto, "Technological diversification and firm's techno-economic structure: An assessment of Canon's sustainable growth trajectory," Technol. Forecast. Soc. Change, vol. 72, no. 1, pp. 11-27, 2005.

[102] S.-H. Chen, M.-H. Huang, D.-Z. Chen, and S.-Z. Lin, "Detecting the temporal gaps of technology fronts: A case study of smart grid field," Technol. Forecast. Soc. Change, vol. 79, no. 9, pp. 1705-1719, 2012.

[103] M. Epicoco, "Knowledge patterns and sources of leadership: Mapping the semiconductor miniaturization trajectory," Res. Policy, vol. 42, no. 1, pp. 180-195, 2012.

[104] J. L. Funk, "The future of the mobile phone Internet: an analysis of technological trajectories and lead users in the Japanese market," Technol. Soc., vol. 27, no. 1, pp. 69-83, 2005.

[105] R. P. Raven, "Towards alternative trajectories? Reconfigurations in the Dutch electricity regime," Res. Policy, vol. 35, no. 4, pp. 581-595, 2006.

[106] F. Castellacci, "Technological paradigms, regimes and trajectories: Manufacturing and service industries in a new taxonomy of sectoral patterns of innovation," Res. Policy, vol. 37, no. 6-7, pp. 978-994, 2008.

[107] D. E. Kash and R. W. Rycoft, "Patterns of innovating complex technologies: a framework for adaptive network strategies," Res. Policy, vol. 29, no. 7-8, pp. 819$831,2000$.

[108] P. Arqué-Castells, "How venture capitalists spur invention in Spain: Evidence from patent trajectories," Res. Policy, vol. 41, no. 5, pp. 897-912, 2012.

[109] W.-J. Kim, J.-D. Lee, and T.-Y. Kim, "Demand forecasting for multigenerational products combining discrete choice and dynamics of diffusion under technological trajectories," Technol. Forecast. Soc. Change, vol. 72, no. 7, pp. 825-849, 2005.

[110] C.-Y. Lee, J.-D. Lee, and Y. Kim, "Demand forecasting for new technology with a short history in a competitive environment: the case of the home networking market in South Korea," Technol. Forecast. Soc. Change, vol. 75, no. 1, pp. 91106, 2008.

[111] V. Krishnan and K. T. Ulrich, "Product development decisions: a review of the literature," Management Science, vol. 47, no. 1. pp. 1-21, 2001. 
[112] M. V. Mansfield and Wagner. K., "Organizational and strategic factors associated with probabilities of success in industrial R\&D," J. Bus., vol. 48, pp. 179-198, 1975.

[113] G. Bacon, S. Beckman, D. C. Mowery, and E. Wilson, "Managing product definition in high-technology industries: a pilot study," Calif. Manage. Rev., vol. 36, no. 2, pp. 32-56, 1994.

[114] V. Srinivasan, W. S. Lovejoy, and D. Beach, "Integrated product design for marketability and manufacturing," J. Mark. Res., vol. 34, no. 1, pp. 154-163, 1997.

[115] E. Dahan and V. Srinivasan, "Predictive power of Internet-based product concept testing using visual depiction and animation," J. Prod. Innov. Manag., vol. 17, no. 2, pp. 99-109, 2000.

[116] A. Schulze and M. Hoegl, "Organizational knowledge creation and the generation of new product ideas: A behavioral approach," Res. Policy, vol. 37, no. 10, pp. 1742-1750, 2008.

[117] J. Kim and D. Wilemon, "Focusing the fuzzy front-end in new product development," R\&D Manag., vol. 32, no. 4, pp. 269-279, 2002.

[118] M. Engwall and A. Jerbrant, "The resource allocation syndrome: the prime challenge of multi-project management?," Int. J. Proj. Manag., vol. 21, no. 6, pp. 403-409, Aug. 2003.

[119] G. R. Schirr, "Flawed tools: the efficacy of group research methods to generate customer ideas," in Journal of Product Innovation Management, 2012, vol. 29, no. 3, pp. 473-488.

[120] N. Dalkey and O. Helmer, "An Experimental Application of the DELPHI Method to the Use of Experts," Manage. Sci., vol. 9, no. 3, pp. 458-467, 1963.

[121] F. Zwicky, "Morphology of propulsive power," Society for Morphological Research, California, pp. 1-382, 1962.

[122] E. von Hippel, "Lead users: a source of novel product concepts," Management Science, vol. 32, no. 7. pp. 791-805, 1986.

[123] A. Griffin and J. Hauser, "The voice of the customer," Mark. Sci., vol. 12, no. 1, pp. 1-27, 1993. 
[124] G. S. Lynn, J. G. Morone, and A. S. Paulson, "Marketing and discontinuous innovation: the probe and learn process," Calif. Manage. Rev., vol. 38, no. 3, pp. 8-37, 1996.

[125] D. Leonard and J. F. Rayport, "Spark innovation through empathic design.," Harv. Bus. Rev., vol. 75, no. 6, pp. 102-113, 1997.

[126] A. J. M. Jetter, "Educating the guess: strategies, concepts and tools for the fuzzy front end of product development," in PICMET-Technology Management for Reshaping the World, 2003, pp. 261-273.

[127] J. Surowiecki, The Wisdom of Crowds. New York: Anchor, 2005, p. 336.

[128] R. Sethi, S. Pant, and A. Sethi, "Web-based product development systems integration and new product outcomes: A conceptual framework," J. Prod. Innov. Manag., vol. 20, no. 1, pp. 37-56, 2003.

[129] M. Fisher, K. Ramdas, and K. Ulrich, "Component sharing in the management of product variety: a study of automotive braking systems," Manage. Sci., vol. 45, no. 3, pp. 297-315, 1999.

[130] S. L. Brown and K. M. Eisenhardt, "Product development: past research, present findings, and future directions," Acad. Manag. Rev., vol. 20, no. 2, pp. 343-378, 1995.

[131] S. Ahmad, D. N. Mallick, and R. G. Schroeder, "New product development: Impact of project characteristics and development practices on performance," $J$. Prod. Innov. Manag., vol. 30, no. 2, pp. 331-348, 2013.

[132] C. Moorman and A. S. Miner, "The impact of organizational memory on new product performance and creativity," J. Mark. Res., vol. 34, no. 1, pp. 91-106, 1997.

[133] L. Cassia, A. De Massis, and E. Pizzurno, "Strategic innovation and new product development in family firms: An empirically grounded theoretical framework," Int. J. Entrep. Behav. Res., vol. 18, no. 2, pp. 198-232, 2012.

[134] E. Hultink, "Industrial new product launch strategies and product development performance," J. Prod. Innov. Manag., vol. 14, no. 4, pp. 243-257, 1997.

[135] Y. Yin, S. Qin, and R. Holland, "Development of a design performance measurement matrix for improving collaborative design during a design process," Int. J. Product. Perform. Manag., vol. 60, no. 2, pp. 152-184, 2011. 
[136] M. Perkmann, A. Neely, and K. Walsh, "How should firms evaluate success in university-industry alliances? A performance measurement system," $R \& D$ Manag., vol. 41, no. 2, pp. 202-216, 2011.

[137] M. D. Morelli, S. D. Eppinger, and R. K. Gulati, "Predicting technical communication in product development organizations," IEEE Trans. Eng. Manag., vol. 42, no. 3, pp. 215-222, 1995.

[138] S. B. Modi and V. A. Mabert, "Supplier development: Improving supplier performance through knowledge transfer," J. Oper. Manag., vol. 25, no. 1, pp. 42 64, 2007.

[139] R. J. Slotegraaf and K. Atuahene-Gima, "Product development team stability and new product advantage: the role of decision-making processes," J. Mark., vol. 75, no. 1, pp. 96-108, 2011.

[140] D. Robertson and T. J. Allen, "CAD system use and engineering performance," IEEE Trans. Eng. Manag., vol. 40, no. 3, pp. 274-282, 1993.

[141] M. Muethel, F. Siebdrat, and M. Hoegl, "When do we really need interpersonal trust in globally dispersed new product development teams?," $R \& D$ Manag., vol. 42, no. 1, pp. 31-46, 2012.

[142] J. E. Burroughs, D. W. Dahl, C. P. Moreau, A. Chattopadhyay, and G. J. Gorn, "Facilitating and rewarding creativity during new product development," J. Mark., vol. 75, no. 4, pp. 53-67, 2011.

[143] N. Ale Ebrahim, S. Ahmed, and Z. Taha, "Modified stage-gate: a conceptual model of virtual product development process," African J. Mark. Manag. Vol 1 No 9 pp 211219 December 2009, vol. 1, no. 9, pp. 211-219, 2009.

[144] K. B. Kahn, G. Barczak, J. Nicholas, A. Ledwith, and H. Perks, "An examination of new product development best practice," J. Prod. Innov. Manag., vol. 29, no. 2, pp. 180-192, 2012.

[145] R. G. Cooper and E. J. Kleinschmidt, "Winning Businesses in Product Devhopmbiit : the Critical Success Factors," Res. Technol. Manag., vol. 50, no. 3, pp. 52-66, 2007.

[146] A. C. Edmondson and I. M. Nembhard, "Product development and learning in project teams: The challenges are the benefits," J. Prod. Innov. Manag., vol. 26, no. 2, pp. 123-138, 2009. 
[147] R. T. Keller, “Transformational leadership, initiating structure, and substitutes for leadership: a longitudinal study of research and development project team performance.," J. Appl. Psychol., vol. 91, no. 1, pp. 202-210, 2006.

[148] R. Pike and S. Ho, "Risk analysis in capital budgeting: barriers and benefits," Omega, vol. 19, no. 4, pp. 235-245, 1991.

[149] N. . Archer and F. Ghasemzadeh, "An integrated framework for project portfolio selection," Int. J. Proj. Manag., vol. 17, no. 4, pp. 207-216, 1999.

[150] N. R. Joglekar and D. N. Ford, "Product development resource allocation with foresight," Eur. J. Oper. Res., vol. 160, no. 1, pp. 72-87, 2005.

[151] K. J. Petersen, R. B. Handfield, and G. L. Ragatz, "Supplier integration into new product development: Coordinating product, process and supply chain design," $J$. Oper. Manag., vol. 23, no. 3-4, pp. 371-388, 2005.

[152] J. Jiao, T. W. Simpson, Z. Siddique, and J. Jiao, "Product family design and platform-based product development: a state-of-the-art review," J. Intell. Manuf., vol. 18, no. 1, pp. 5-29, 2007.

[153] X. A. Koufteros, T. C. Edwin Cheng, and K. H. Lai, “'Black-box' and 'gray-box' supplier integration in product development: Antecedents, consequences and the moderating role of firm size," J. Oper. Manag., vol. 25, no. 4, pp. 847-870, 2007.

[154] D. B. Hertz and H. Thomas, Risk analysis and its applications. New York: Wiley, 1983, p. 340.

[155] D. H. Stamatis, Failure mode and effect analysis: FMEA from theory to execution, vol. 38. 2003, p. 80.

[156] J. A. Keizer, J. P. Vos, and J. I. M. Halman, "Risks in new product development: Devising a reference tool," R\&D Manag., vol. 35, no. 3, pp. 297-309, 2005.

[157] T. Markeset and U. Kumar, "Integration of RAMS information in design processes-a case study," in Annual Reliability and Maintainability Symposium, 2003, pp. 220-225.

[158] P. E. Green and V. Srinivasan, "Conjoint analysis in consumer research: issues and outlook," J. Consum. Res., vol. 5, no. 2, p. 103, 1978.

[159] G. R. Amin, A. Emrouznejad, and S. Rezaei, "Some clarifications on the DEA clustering approach,” Eur. J. Oper. Res., vol. 215, no. 2, pp. 498-501, 2011. 
[160] G. Rowe and G. Wright, "The Delphi technique as a forecasting tool: issues and analysis," Int. J. Forecast., vol. 15, no. 4, pp. 353-375, 1999.

[161] M. T. A. Wentholt, S. Cardoen, H. Imberechts, X. Van Huffel, B. W. Ooms, and L. J. Frewer, "Defining European preparedness and research needs regarding emerging infectious animal diseases: Results from a Delphi expert consultation," Prev. Vet. Med., vol. 103, no. 2-3, pp. 81-92, 2012.

[162] J. Pearce, C. Jones, S. Morrison, M. Olff, S. van Buschbach, A. B. Witteveen, R. Williams, F. Orengo-García, D. Ajdukovic, A. T. Aker, D. Nordanger, B. LuegerSchuster, and J. I. Bisson, "Using a delphi process to develop an effective trainthe-trainers program to train health and social care professionals throughout Europe," J. Trauma. Stress, vol. 25, no. 3, pp. 337-343, 2012.

[163] J. Landeta and J. Barrutia, "People consultation to construct the future: A Delphi application," Int. J. Forecast., vol. 27, no. 1, pp. 134-151, 2011.

[164] O. Lindqvist, G. Lundquist, A. Dickman, J. Bükki, U. Lunder, C. L. Hagelin, B. H. Rasmussen, S. Sauter, C. Tishelman, and C. J. Fürst, "Four essential drugs needed for quality care of the dying: a Delphi-study based international expert consensus opinion.," J. Palliat. Med., vol. 16, no. 1, pp. 38-43, 2013.

[165] H. Kahn, “On alternative world futures: issues and themes,” New York, 1965.

[166] M. Nowack, J. Endrikat, and E. Guenther, "Review of Delphi-based scenario studies: Quality and design considerations," Technol. Forecast. Soc. Change, vol. 78, no. 9, pp. 1603-1615, 2011.

[167] J. J. Winebrake and B. P. Creswick, "The future of hydrogen fueling systems for transportation: An application of perspective-based scenario analysis using the analytic hierarchy process," Technol. Forecast. Soc. Change, vol. 70, no. 4, pp. 359-384, 2003.

[168] K. Kok, M. van Vliet Mathijs, I. Bärlund Ilona, A. Dubel, and J. Sendzimir, "Combining participative backcasting and exploratory scenario development: Experiences from the SCENES project," Technol. Forecast. Soc. Change, vol. 78, no. 5, pp. 835-851, 2011.

[169] A. Jetter and W. Schweinfort, "Building scenarios with Fuzzy Cognitive Maps: An exploratory study of solar energy,” Futures, vol. 43, no. 1, pp. 52-66, 2011.

[170] T. A. Tran and T. Daim, "A taxonomic review of methods and tools applied in technology assessment," Technol. Forecast. Soc. Change, vol. 75, no. 9, pp. 13961405, 2008. 
[171] I. Linkov, F. K. Satterstrom, G. Kiker, C. Batchelor, T. Bridges, and E. Ferguson, "From comparative risk assessment to multi-criteria decision analysis and adaptive management: recent developments and applications.," Environ. Int., vol. 32, no. 8, pp. 1072-93, 2006.

[172] C. S. Kolar and D. M. Lodge, "Ecological predictions and risk assessment for alien fishes in North America," Science, vol. 298, no. 5596, pp. 1233-1236, 2002.

[173] P. Morris and R. Therivel, Methods of Environmental Impact Assessment, vol. 2, no. 3. Spon Press, 2001, p. 378.

[174] R. Ramanathan, "A note on the use of the analytic hierarchy process for environmental impact assessment.," J. Environ. Manage., vol. 63, no. 1, pp. 27-35, 2001.

[175] H. M. G. Van Der Werf and J. Petit, "Evaluation of the environmental impact of agriculture at the farm level: A comparison and analysis of 12 indicator-based methods," Agric. Ecosyst. Environ., vol. 93, no. 1-3, pp. 131-145, 2002.

[176] C. W. Churchman, R. L. Ackoff, and E. L. Arnoff, Introduction to Operations Research. New York: John Wiley \& Sons, 1957.

[177] D. Ghiculescu, N. Marinescu, D. Ghiculescu, and C. Pirnău, "Customer matrix with different time horizons applied to electrodischarge machines," Appl. Mech. Mater., vol. 371, pp. 220-224, 2013.

[178] G. Manuel and J. Pretorius, "The significance of relevance trees in the identification of artificial neural networks input vectors," J. Energy South. Africa, vol. 24, no. 1, pp. 27-34, 2013.

[179] X. Feng and L. Fuhai, "Patent text mining and informetric-based patent technology morphological analysis: an empirical study," Technol. Anal. Strateg. Manag., vol. 24, no. 5, pp. 467-479, 2012.

[180] S. Jun, S. S. Park, and D. S. Jang, "Technology forecasting using matrix map and patent clustering," Ind. Manag. Data Syst., vol. 112, no. 5, pp. 786-807, 2012.

[181] B. Yoon, I. Park, and B. youl Coh, "Exploring technological opportunities by linking technology and products: Application of morphology analysis and text mining," Technol. Forecast. Soc. Change, vol. 86, pp. 287-303, 2014.

[182] B. Mazlish, The railroad and the space program - An exploration in historical analogy. Cambridge: The MIT press, 1965, pp. 1-223. 
[183] P. Goodwin, K. Dyussekeneva, and S. Meeran, "The use of analogies in forecasting the annual sales of new electronics products," IMA J. Manag. Math., vol. 24, no. 4, pp. 407-422, 2012.

[184] S. C. Bhargava, "A generalized form of the fisher-pry model of technological substitution,” Technol. Forecast. Soc. Change, vol. 49, no. 1, pp. 27-33, 1995.

[185] K. Christodoulou, K. Jensen, and K. Vlahos, "Using object-oriented simulation to explore substitution between technologies: An application to the UK mobile telecommunications industry," Technol. Forecast. Soc. Change, vol. 62, no. 3, pp. 203-217, 1999.

[186] M. Dekimpe, P. Parker, and M. Sarvary, “'Globalization': modeling technology adoption timing across countries," Technol. Forecast. Soc. Change, vol. 63, no. 1, pp. $25-42,2000$.

[187] J. Goldenberg and S. Efroni, "Using cellular automata modeling of the emergence of innovations," Technol. Forecast. Soc. Change, vol. 68, no. 3, pp. 293-308, 2001.

[188] T. Islam and N. Meade, "The diffusion of successive generations of a technology: A more general model," Technol. Forecast. Soc. Change, vol. 56, no. 1, pp. 49-60, 1997.

[189] D. M. Kiefer, "Winds of change in industrial chemical research," Chem. Eng. News, vol. 42, no. 23, pp. 88-109, 1964.

[190] W. H. Pound, "Research project selection: Testing a model in the field," IEEE Trans. Eng. Manag., vol. EM-11, no. 1, pp. 16-22, 1964.

[191] R. E. Beckwith, "A stochastic regression model for proposal success evaluation," IEEE Trans. Eng. Manag., vol. EM-12, no. 2, pp. 59-62, 1965.

[192] J. Ondrus and Y. Pigneur, "An assessment of NFC for future mobile payment systems," in International Conference on the Management of Mobile Business ICMB 2007, 2007, pp. 43-43.

[193] T. U. Daim, E. Bayraktaroglu, J. Estep, D. J. Lim, J. Upadhyay, and J. Yang, "Optimizing the NW off-shore wind turbine design," Math. Comput. Model., vol. 55, no. 3, pp. 396-404, 2011.

[194] M. T. Tang and G.-H. Tzeng, "A hierarchy fuzzy MCDM method for studying electronic marketing strategies in the information service industry," in International Academy for Information Management Annual Conference, 1998, no. 1, pp. 1-16. 
[195] B. V. Dean and S. S. Sengupta, "Research budgeting and project selection," IRE Trans. Eng. Manag., vol. 4, pp. 158-169, 1962.

[196] K. Moslehi and R. Kumar, "A reliability perspective of the smart grid," IEEE Trans. Smart Grid, vol. 1, no. 1, pp. 57-64, 2010.

[197] M. Friedewald and O. Raabe, "Ubiquitous computing: An overview of technology impacts," Telemat. Informatics, vol. 28, no. 2, pp. 55-65, 2011.

[198] B. J. W. Forrester, "Industrial dynamics. A major breakthrough for decision makers," Harv. Bus. Rev., vol. 36, no. 4, pp. 37-66, 1958.

[199] T. U. Daim, G. Rueda, H. Martin, and P. Gerdsri, "Forecasting emerging technologies: Use of bibliometrics and patent analysis," Technol. Forecast. Soc. Change, vol. 73, no. 8, pp. 981-1012, 2006.

[200] F. H. Maier, "New product diffusion models in innovation management-a system dynamics perspective," Syst. Dyn. Rev., vol. 14, pp. 285-308, 1998.

[201] E. Suryani, S. Y. Chou, and C. H. Chen, "Air passenger demand forecasting and passenger terminal capacity expansion: A system dynamics framework," Expert Syst. Appl., vol. 37, no. 3, pp. 2324-2339, 2010.

[202] J. Martino, "A comparison of two composite measures of technology," Technol. Forecast. Soc. Change, vol. 44, no. 2, pp. 147-159, 1993.

[203] N. Gerdsri, "An Analytical Approach on Building a Technology Development Envelope (TDE) for Roadmapping of Emerging Technologies," Portland State University, 2005.

[204] A. J. Alexander and J. R. Nelson, "Measuring technological change: Aircraft turbine engines," Technol. Forecast. Soc. Change, vol. 5, no. 2, pp. 189-203, 1973.

[205] D.-J. Lim, T. R. Anderson, and J. Kim, "Forecast of wireless communication technology: A comparative study of regression and TFDEA Model," in PICMETTechnology Management for Emerging Technologies, 2012, pp. 1247-1253.

[206] G. C. Taylor, "Methodologies for characterizing technologies," Electric Power Research Institute, Palo Alto, p. 120, 1978.

[207] R. E. Klitgaard, "Measuring technological change: Comments on a proposed methodology," Technol. Forecast. Soc. Change, vol. 6, pp. 437-440, 1974. 
[208] D. J. Aigner and S. F. Chu, "On estimating the industry production function," Am. Econ. Rev., vol. 58, no. 4, pp. 826-839, 1968.

[209] H. O. Fried, C. A. K. Lovell, and S. S. Schmidt, The Measurement of Productive Efficiency and Productivity Growth, 1st ed. New York: Oxford University Press, 2008, p. 638.

[210] D. Aigner, C. A. K. Lovell, and P. Schmidt, "Formulation and estimation of stochastic frontier production function models," J. Econom., vol. 6, no. 1, pp. 2137, 1977.

[211] E. N. Dodson, "Measurement of state of the art and technological advance," Technol. Forecast. Soc. Change, vol. 146, no. 2-3, pp. 129-146, 1985.

[212] E. N. Dodson, "A general approach to measurement of the state of the art and technological advance," Technol. Forecast. Soc. Change, vol. 1, no. 4, pp. 391$408,1970$.

[213] J. P. Martino, "Measurement of technology using tradeoff surfaces," Technol. Forecast. Soc. Change, vol. 27, no. 2, pp. 147-160, 1985.

[214] B. F. Cole, "An Evolutionary Method for Synthesizing Technological Planning and Architectural Advance," Georgia Institute of Technology, 2009.

[215] M. J. Farrell, “The measurement of productive efficiency," J. R. Stat. Soc. Ser. A, vol. 120, no. 3, pp. 253-290, 1957.

[216] A. Charnes, W. W. Cooper, and E. Rhodes, "Measuring the efficiency of decision making units," Eur. J. Oper. Res., vol. 2, no. 6, pp. 429-444, 1978.

[217] R. Fare, S. Grosskopf, and C. A. K. Lovell, Production Frontiers. Cambridge University Press, 1994, p. 316.

[218] A. Charnes, C. T. Clark, W. W. Cooper, and B. Golany, "A developmental study of data envelopment analysis in measuring the efficiency of maintenance units in the U.S. air forces," Annals of Operations Research, vol. 2, no. 1. pp. 95-112, 1984.

[219] T. Sueyoshi, "Comparisons and analyses of managerial efficiency and returns to scale of telecommunication enterprises by using DEA/WINDOW," Commun. Oper. Res. Soc. Japan, vol. 37, pp. 210-219, 1992. 
[220] W. W. Cooper, L. M. Seiford, and K. Tone, Introduction to Data Envelopment Analysis and Its Uses: with DEA-solver Software and References. Springer New York, 2006, p. 354.

[221] A. Emrouznejad and E. Thanassoulis, "Measurement of productivity index with dynamic DEA,” Int. J. Oper. Res., vol. 8, no. 2, pp. 247-260, 2010.

[222] S. Malmquist, "Index numbers and indifference surfaces," Trab. Estad., vol. 4, no. 2, pp. 209-242, 1953.

[223] O. L. Inman, "Technology Forecasting using Data Envelopment Analysis," Portland State University, 2004.

[224] J. O. Kim, J. H. Kim, and S. K. Kim, “A Hybrid Technological Forecasting Model by Identifying the Efficient DMUs: An Application to the Main Battle Tank," Asian J. Technol. Innov., vol. 15, no. 2, pp. 83-102, 2007.

[225] A.-M. Lamb, T. R. Anderson, and T. Daim, "Research and development targetsetting difficulties addressed through the emergent method: technology forecasting using data envelopment analysis," $R \& D$ Manag., vol. 42, no. 4, pp. 327-341, 2012.

[226] K. C. Land, C. A. K. Lovell, and S. Thore, "Chance-constrained data envelopment analysis," Manag. Decis. Econ., vol. 14, no. 6, pp. 541-554, 1993.

[227] P. L. Brockett, A. Charnes, W. W. Cooper, Z. M. Huang, and D. B. Sun, "Data transformations in DEA cone ratio envelopment approaches for monitoring bank performances," Eur. J. Oper. Res., vol. 98, no. 2, pp. 250-268, 1997.

[228] A. Charnes and W. W. Cooper, "Chance-constrained programming," Manage. Sci., vol. 6, no. 1, pp. 73-79, 1959.

[229] J. K. Sengupta, "Transformations in stochastic DEA models," J. Econom., vol. 46, no. 1, pp. 109-123, 1990.

[230] O. Inman, T. R. Anderson, and R. Harmon, "Predicting U.S. jet fighter aircraft introductions from 1944 to 1982: A dogfight between regression and TFDEA," Technol. Forecast. Soc. Change, vol. 73, no. 9, pp. 1178-1187, 2006.

[231] T. R. Anderson, R. Fare, S. Grosskopf, L. Inman, and X. Song, "Further examination of Moore's law with data envelopment analysis," Technol. Forecast. Soc. Change, vol. 69, no. 5, pp. 465-477, 2002. 
[232] D.-J. Lim, N. Runde, and T. R. Anderson, "Applying technology forecasting to new product development target setting of LCD panels," in Advances in Business and Management Forecasting, 9th ed., Emerald, 2013, p. 340.

[233] W. W. Cooper, L. M. Seiford, and K. Tone, Data Envelopment Analysis: A Comprehensive Text with Models, Applications, References and DEA-solver Software. New York: Springer, 2007, p. 512.

[234] R. D. Banker, A. Charnes, and W. W. Cooper, "Some models for estimating technical and scale inefficiencies in data envelopment analysis," Management, vol. 30, no. 9, pp. 1078-1092, 1984.

[235] C. A. K. Lovell and A. P. B. Rouse, "Equivalent standard DEA models to provide super-efficiency scores," J. Oper. Res. Soc., vol. 54, no. 1, pp. 101-108, 2003.

[236] W. D. Cook, L. Liang, Y. Zha, and J. Zhu, "A modified super-efficiency DEA model for infeasibility,” J. Oper. Res. Soc., vol. 60, no. 2, pp. 276-281, 2009.

[237] H.-S. Lee, C.-W. Chu, and J. Zhu, "Super-efficiency DEA in the presence of infeasibility,” Eur. J. Oper. Res., vol. 212, no. 1, pp. 141-147, 2011.

[238] H.-S. Lee and J. Zhu, "Super-efficiency infeasibility and zero data in DEA," Eur. J. Oper. Res., vol. 216, no. 2, pp. 429-433, 2012.

[239] P. Andersen and N. C. Petersen, "A procedure for ranking efficient units in data envelopment analysis," Manage. Sci., vol. 39, no. 10, pp. 1261-1264, 1993.

[240] D.-J. Lim, T. R. Anderson, and O. L. Inman, "Choosing effective dates from multiple optima in Technology Forecasting using Data Envelopment Analysis (TFDEA)," Technol. Forecast. Soc. Change, vol. 88, pp. 91-97, 2014.

[241] A. A. Ali and L. M. Seiford, "Computational accuracy and infinitesimals in data envelopment analysis," INFOR, vol. 31, no. 4, pp. 290-297, 1993.

[242]A.-M. Lamb, T. U. Daim, and T. R. Anderson, "Forecasting airplane technologies," Foresight, vol. 12, no. 6. pp. 38-54, 2010.

[243] D. A. Irwin and N. Pavcnik, "Airbus versus Boeing revisited: international competition in the aircraft market," J. Int. Econ., vol. 64, no. 2, pp. 223-245, 2004.

[244] Anonymous, “Technical charateristics - Boeing 747-400,” Boeing, 2013.

[245] D. L. Greene, "Energy-efficiency improvement potential of commercial aircraft," Annu. Rev. Energy Environ., vol. 17, no. 1, pp. 537-573, 1992. 
[246] D. Briere and P. Traverse, AIRBUS A320/A330/A340 electrical flight controls - A family of fault-tolerant systems. IEEE Comput. Soc. Press, 1993, pp. 616-623.

[247] Anonymous, "777-200LR/-300ER/-freighter airplane characteristics for airport planning," Boeing, Boeing, pp. 1-166, 2009.

[248] J. Michaels, "The Airbus A380: The giant on the runway," The Economist, vol. 385, no. 8550, pp. 81-83, 2007.

[249] M. Berrittella, L. La Franca, V. Mandina, and P. Zito, "Modelling strategic alliances in the wide-body long-range aircraft market," J. Air Transp. Manag., vol. 13, no. 3, pp. 139-148, 2007.

[250] J. C. Williams and E. A. Starke, "Progress in structural materials for aerospace systems," Acta Mater., vol. 51, no. 19, pp. 5775-5799, 2003.

[251] D. A. Senzig, G. G. Fleming, and R. J. Iovinelli, "Modeling of terminal-area airplane fuel consumption," J. Aircr., vol. 46, no. 4, pp. 1089-1093, 2009.

[252] Anonymous, “Technical characteristics - Boeing 747-8," Boeing, 2013. [Online]. Available: http://www.boeing.com/boeing/commercial/747family/7478_fact_sheet.page.

[253] W. Steve, "Reports hint at what may be behind Boeing 747-8 delay," Puget Sound Business Journal, 2011. [Online]. Available: http://www.bizjournals.com/seattle/news/2011/09/16/boeing-monday-747-8freighter-delivery.html?page $=2$.

[254] S. Mary and R. Susanna, "Boeing affirms delivery target for first 787-9 Dreamliner," Bloomberg, 2012. [Online]. Available: http://www.bloomberg.com/news/articles/2012-10-17/american-says-first-boeing787-9-dreamliner-delayed-beyond-2014.

[255] M. Harriet, "Boeing delays decision on 787-10 production site," Reuters, 2014. [Online]. Available: http://www.reuters.com/article/2014/03/10/us-boeingsouthcarolina-idUSBREA291LA20140310.

[256] G. Dominic, "Simmering Boeing strike scorching both sides," The Seattle Times, 2008. [Online]. Available: http://www.seattletimes.com/business/boeingaerospace/simmering-boeing-strike-scorching-both-sides.

[257] R. Andrea and B. Thomas, "Airbus blinking with A350 helps Boeing plot 777 successor," Bloomberg, 2012. [Online]. Available: 
http://www.bloomberg.com/news/articles/2012-05-29/airbus-blinking-first-witha350-helps-boeing-plot-777-successor.

[258] H. Scott, "Smaller seats, fee rises and new planes? 2014: The year ahead in air travel," CNN, 2014. [Online]. Available:

http://www.cnn.com/2014/01/01/travel/2014-aviation-year/.

[259] K. Max, "PICTURE: 10-abreast A350 XWB 'would offer unprecedented operating cost advantage,"” Flight, 2008. [Online]. Available:

http://www.flightglobal.com/news/articles/picture-10-abreast-a350-xwb-39wouldoffer-unprecedented-operating-cost-223853/

[260] Anonymous, “A350XWB family A350-1000,” Airbus, 2014. [Online]. Available: http://www.airbus.com/aircraftfamilies/passengeraircraft/a350xwbfamily/a350$1000 /$.

[261] L. J. Tashman, “Out-of-sample tests of forecasting accuracy: an analysis and review," Int. J. Forecast., vol. 16, no. 4, pp. 437-450, 2000.

[262] S. Makridakis, S. C. Wheelwright, and R. J. Hyndman, Forecasting: Methods and Applications, 8th ed. New York: John Wiley \& Sons, 2008, p. 656.

[263] J. S. Armstrong, Principles of Forecasting: A Handbook for Researchers and Practitioners, 2001st ed. New York: Springer, 2001, p. 849.

[264]R. J. Hyndman and A. B. Koehler, "Another look at measures of forecast accuracy," Int. J. Forecast., vol. 22, no. 4, pp. 679-688, 2006.

[265] S. Makridakis and R. Winkler, "Sampling distributions of post-sample forecasting errors," Appl. Stat., vol. 38, no. 2, pp. 331-342, 1989.

[266] N. R. Swanson and H. White, "Forecasting economic time series using flexible versus fixed specification and linear versus nonlinear econometric models," Int. J. Forecast., vol. 13, no. 4, pp. 439-461, 1997.

[267] J. S. Armstrong and F. Collopy, "Error measures for generalizing about forecasting methods: Empirical comparisons," Int. J. Forecast., vol. 8, no. 1, pp. 69-80, 1992.

[268] S. Jahromi, T. Anderson, and A. Tudori, "Forecasting hybrid electric vehicles using TFDEA," in PICMET-Technology Management in the IT-Driven Services, 2013, pp. 2098-2107.

[269] B. S. Yoon, A. Charoensupyan, N. Hu, R. Koosawangsri, M. Abdulai, and X. Wang, "Digital single-lens reflex camera technology forecasting with technology 
forecasting using data envelopment analysis," in PICMET-Technology Management in the IT-Driven Services, 2013.

[270] D. J. Lim and D. F. Kocaoglu, "China - can it move from imitation to innovation?," in PICMET-Technology Management in the Energy Smart World, pp. 1-13.

[271] D. G. Feitelson, "The supercomputer industry in light of the Top500 data," Comput. Sci. Eng., vol. 7, no. 1, pp. 42-47, 2005.

[272] J. Dongarra, "Visit to the national university for defense technology changsha, china," Oak Ridge National Laboratory, pp. 1-18, 2013.

[273] National Research Council, Getting Up to Speed: The Future of Supercomputing, 1st ed. Washington, D.C: National Academies Press, 2005, p. 308.

[274] J. Dongarra, H. Meuer, and E. Strohmaier, "TOP500 supercomputer sites," Supercomputer, vol. 13, pp. 89-111, 1997.

[275] M. Peckham, "RIKEN plans Exascale supercomputer '30 times faster' than today's fastest in six years," Time, New York, 2013.

[276] S. Ashby, P. Beckman, J. Chen, P. Colella, B. Collins, D. Crawford, J. Dongarra, D. Kothe, R. Lusk, P. Messina, T. Mezzacappa, P. Moin, M. Norman, R. Rosner, V. Sarkar, A. Siegel, F. Streitz, A. White, and M. Wright, "The opportunities and challenges of Exascale computing," U.S Department of Energy (Office of Science), pp. 1-71, 2010.

[277] H. Simon, T. Zacharia, and R. Stevens, "Modeling and simulation at the Exascale for energy and the environment," Dep. Energy Tech. Rep., 2007.

[278] S. Kamil, J. Shalf, and E. Strohmaier, "Power efficiency in high performance computing," in 2008 IEEE International Symposium on Parallel and Distributed Processing, 2008, pp. 1-8.

[279] C.-H. Hsu, J. A. Kuehn, and S. W. Poole, "Towards efficient supercomputing: A quest for the right metric," in The third joint WOSP/SIPEW international conference on Performance Engineering, 2012, p. 157.

[280] F. Robert, "Who will step up to exascale?," Science, pp. 264-266, 2013.

[281] T. Geller, "Supercomputing's exaflop target," Commun. ACM, vol. 54, no. 8, p. 16, 2011. 
[282] H. Simon, "Why we need exascale and why we won't get there by 2020," in Optical Interconnects Conference, 2013.

[283] J. D. Owens, M. Houston, D. Luebke, S. Green, J. E. Stone, and J. C. Phillips, "GPU computing," Proc. IEEE, vol. 96, no. 5, pp. 879-899, May 2008.

[284] Sheng Li, J.-H. Ahn, R. D. Strong, J. B. Brockman, D. M. Tullsen, and N. P. Jouppi, "McPAT: An integrated power, area, and timing modeling framework for multicore and manycore architectures," in MICRO-42. 42nd Annual IEEE/ACM International Symposium on. IEEE, 2009, pp. 469-480.

[285] J. P. Panziera, "Five challenges for future supercomputers," Supercomputers, pp. $38-40,2012$.

[286] R. Vuduc and K. Czechowski, "What GPU computing means for high-end systems," IEEE Micro, pp. 74-78, 2011.

[287] N. Rajovic, P. Carpenter, I. Gelado, N. Puzovic, and A. Ramirez, "Are mobile processors ready for HPC?," in Supercomputing Conference, 2013.

[288] J. J. Dongarra, P. Luszczek, and A. Petitet, "The LINPACK benchmark: past, present and future," Concurr. Comput. Pract. Exp., vol. 15, no. 9, pp. 803-820, 2003.

[289] P. Wood, "Cray Inc. replacing IBM to build UI supercomputer," The News Gazette, Illinois, p. 1, 2011.

[290] L. Lawrence, "Cray jumped from AMD for the flexibility and performance of Intel chips," The Inquirer, 2012.

[291] C. Murariu, "Intel signs deal with Cray, marks AMD's departure from Cray's supercomputers," Softpedia, 2012.

[292] F. Wang, C.-Q. Yang, Y.-F. Du, J. Chen, H.-Z. Yi, and W.-X. Xu, "Optimizing Linpack benchmark on GPU-accelerated Petascale supercomputer," J. Comput. Sci. Technol., vol. 26, no. 5, pp. 854-865, Sep. 2011.

[293] A. Cole, "Intel and InfiniBand: pure HPC play, or is there a fabric in the works?," IT Business Edge, 2012.

[294] Anonymous, "InfiniBand strengthens leadership as the high-speed interconnect of choice," Mellanox Technologies, pp. 1-31, 2012. 
[295] Z. Whittaker, "Intel buys QLogic's InfiniBand assets for \$125 million," ZD Net, 2012. [Online]. Available: http://www.zdnet.com/article/intel-buys-qlogicsinfiniband-assets-for-125-million/.

[296] IntelPR, "Intel acquires industry-leading, high-performance computing interconnect technology and expertise," Intel Newsroom, 2012. [Online]. Available: http://newsroom.intel.com/community/intel_newsroom/blog/2012/04/24/intelacquires-industry-leading-high-performance-computing-interconnect-technologyand-expertise.

[297] T. Morgan, "Intel came a-knockin' for Cray super interconnects," The Register, 2012. [Online]. Available:

http://www.theregister.co.uk/2012/04/25/intel_cray_interconnect_followup/.

[298] R. Haring, M. Ohmacht, T. Fox, M. Gschwind, P. Boyle, N. Chist, C. Kim, D. Satterfield, K. Sugavanam, P. Coteus, P. Heidelberger, M. Blumrich, R. Wisniewski, A. Gara, and G. Chiu, "The IBM Blue Gene/Q compute chip," IEEE Micro, vol. 32, no. 2, pp. 48-60, 2011.

[299] C. Lazou, "Should I buy GPGPUs or Blue Gene?," HPCwire, 2010.

[300] M. Feldman, "Blue Genes and GPU clusters top the latest Green500," HPCwire, 2011.

[301] H. Kimihiko, "Exascale supercomputer project," RIKEN Advanced Institute for Computational Science, Kobe, 2013.

[302] L. Tung, "After X86 servers, is IBM gearing up to sell its chips business too?," ZDNet, 2014. [Online]. Available: http://www.zdnet.com/article/after-x86-serversis-ibm-gearing-up-to-sell-its-chips-business-too/.

[303] Davis Nick, "Cray CS300 cluster supercomputers to feature new Intel(R) Xeon Phi(TM) coprocessors x100 family," Cray Media, 2013. [Online]. Available: http://investors.cray.com/phoenix.zhtml?c=98390\&p=irolnewsArticle\&ID=1830424.

[304] Davis Nick, "Cray to acquire appro international," Cray Media, 2012. [Online]. Available: http://investors.cray.com/phoenix.zhtml?c=98390\&p=irolnewsArticle\&ID=1756786.

[305] P. Thibodeau, "Coming by 2023, an exascale supercomputer in the U.S.," Computerworld, 2014. [Online]. Available: http://www.computerworld.com/article/2849250/coming-by-2023-an-exascalesupercomputer-in-the-us.html. 
[306] D. Takahashi, "IBM and Nvidia win $\$ 425 \mathrm{M}$ to build two monstrous supercomputers for the Department of Energy," VentureBeat, 2014.

[307] Anonymous, "Department of energy awards $\$ 425$ million for next generation supercomputing technologies," Department of Energy, 2014.

[308] Y. Kawaguchi, "Japan's policy toward exascale computing,” Ministry of Education, Culture, Sports, Science, and Technology (Japan), 2014.

[309] R. V. Aroca and L. M. G. Gonçalves, "Towards green data centers: A comparison of x86 and ARM architectures power efficiency," J. Parallel Distrib. Comput., vol. 72, no. 12, pp. 1770-1780, 2012.

[310] A. Maruccia, "US intelligence signs up IBM to build an eco-friendly supercomputer," The Inquirer, 2014.

[311] S. Anthony, "US intel agency is developing a superconducting exascale computer and cryogenic memory," Extreme Tech, 2014.

[312] D.-J. Lim and T. R. Anderson, "Technology trajectory mapping using data envelopment analysis: the ex-ante use of disruptive innovation theory on flat panel technologies," R\&D Manag.(In press), 2015.

[313] D.-J. Lim, "Technology forecasting using DEA in the presence of infeasibility," Int. Trans. Oper. Res.(In press), 2015.

[314] A. Charnes, W. W. Cooper, and R. M. Thrall, "A structure for classifying and characterizing efficiency and inefficiency in Data Envelopment Analysis," $J$. Product. Anal., vol. 2, no. 3, pp. 197-237, 1991.

[315] T. R. Anderson and L. Inman, "Resolving the issue of multiple optima in Technology Forecasting using Data Envelopment Analysis," in PICMETTechnology Management in the Energy Smart World, 2011, pp. 1-5.

[316] S. Durairajan, M. I. Prado, N. Rahimi, and S. R. Jahromi, "Forecasting microprocessor technology in the multicore era using TFDEA," in PICMETTechnology Management in the IT-Driven Services, 2013, pp. 2108-2115.

[317] D.-J. Lim and T. R. Anderson, "Improving forecast accuracy by a segmented rate of change in technology forecasting using data envelopment analysis (TFDEA)," in PICMET-Infrastructure and Service Integration, 2014, pp. 2903-2907. 
[318] D.-J. Lim, T. R. Anderson, and T. Shott, "Technological forecasting of supercomputer development: The march to Exascale computing," Omega, vol. 51, pp. 128-135, 2015.

[319] L. Kilian and M. P. Taylor, "Why is it so difficult to beat the random walk forecast of exchange rates?," J. Int. Econ., vol. 60, no. 1, pp. 85-107, 2003. 


\section{Appendix. Model Building Guidelines}

Step 1: Selection of the input(s) and the output(s)

The classification of product features into suitable input(s) and output(s) is difficult, but it is of vital significance. Theoretically, the inputs should capture all resources that significantly affect the outputs. The outputs should reflect all relevant outcomes on which one wishes to assess the technologies. A common pitfall of treating undesirable outputs as inputs should be avoided, and in such a case it is advised to construct the model with weak disposability. Moreover, any environmental factors that directly affect the transformation of inputs into outputs should also be reflected in the model either by including nondiscretionary factors or by normalizing the inputs and/or the outputs.

\section{Step 2: Selection of the orientation}

The orientation selection is purely based upon which direction one wishes to measure the technological progress. For example, the input-oriented model captures the technological progress in a way that it demonstrates how many input reductions have been made while attaining the same levels of outputs, whereas the output-oriented model captures the technological progress in a way that it demonstrates how many output augmentations have been achieved while maintaining the same levels of inputs. Note that non-oriented models could also be implemented by specifying the desired direction of the measurement. Thus, the analyst needs to articulate the purpose of the analysis, whether input reduction, output expansion or both. 
Step 3: Selection of the returns to scale (RTS)

The returns to scale assumptions determine the shape of the frontier by constructing the linear segments based on observed technologies. The simplest form is the constant returns to scale (CRS) assuming the ratio of output(s) to input(s) is not affected by scale size. Therefore, the CRS would be appropriate should the virtual technologies scaled up (or down) from the observed technologies be feasible. The increasing RTS (IRS or NDRS) assumes that it is possible to introduce virtual technologies scaled up but not down from the observed technologies. In contrast, decreasing RTS (DRS or NIRS) introduces virtual technologies scaled down from the observed technologies only. In a situation where virtual technologies generated as a mix of (or scaled from) observed technologies are less convincing as benchmarks than actually observed technologies, free disposal hull (FDH) would be suitable to construct the nonconvex set.

\section{Step 4: Selection of the frontier year (T)}

The frontier year determines the time in which the rate of change is obtained. This may be the most recent time in the dataset when the predictive analysis can benefit from updating the rate of change by up-to-date data or a certain point in time such as a forecasting origin where the time series hold-out sampling begins. In the latter case, the rule of thumb often recommends picking the forecasting origin in such a way that the training period can account for at least two-thirds of the dataset. 
Step 5: Selection of the frontier type and second goal

The current model has two options for the frontier type: static and dynamic. The static frontier fixes the time of the current frontier as a predefined frontier year. That is, the model treats all the technologies on the current frontier as being concurrent in the frontier year. In contrast, the dynamic frontier allows having varying points of time on the current frontier whereby each forecasting target would have a different starting point of forecast. Therefore, the practical decision factor may be influenced by whether the past technologies still located on the current frontier should be regarded as current technologies (in terms of frontier year). This choice necessarily affects the calculation of the rate of change as well as the arrival of the forecasting target since it determines elapsed time of technological progress.

When the dynamic frontier is selected, a secondary objective function should also be specified to choose the individual target year (i.e., effective date) from multiple optima. In particular, the maximization option will choose the benchmark technology presenting in the farthest time horizon by identifying the maximum sum of effective dates, whereas the minimization option will choose the benchmark technology presenting in the closest time horizon by identifying the minimum sum of effective dates. As a result, the maximization (minimization) option tends to compute the rate of change more conservatively (aggressively) but makes more aggressive (conservative) forecasts than the minimization (maximization) option. Note that depending on the application area, slack maximization may be preferred to prevent weakly efficient (non-pareto) technologies from setting the effective date. 